\title{
Trisoxazoline/Cu(II)-promoted Kinugasa Reaction. Enantioselective Synthesis of $\beta$-Lactams
}

\author{
Meng-Chun Ye, Jian Zhou and Yong Tang* \\ State Key Laboratory of Organometallic Chemistry, Shanghai Institute of Organic Chemistry, 354 \\ Fenglin Road, Shanghai 200032, China. \\ tangy@mail.sioc.ac.cn
}

\section{Supporting Information}

\section{Contents}

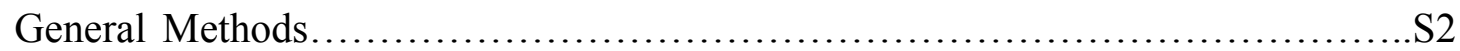

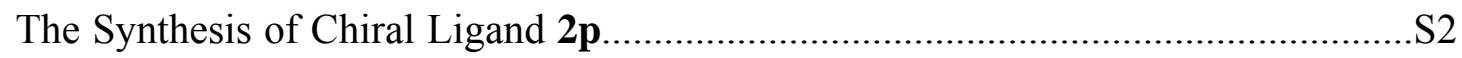

General Procedures for Catalytic Asymmetric Synthesis of $\beta$-Lactams..............S3

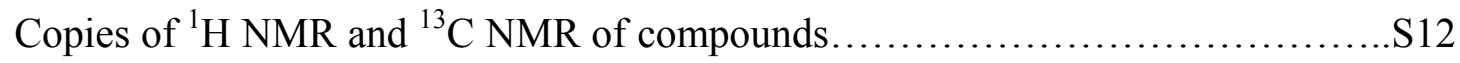

Copies of HPLC spectra of Michael Addition products.........................S34 
General Methods. $\mathrm{Cu}\left(\mathrm{ClO}_{4}\right)_{2} \cdot 6 \mathrm{H}_{2} \mathrm{O}$, alkynes and acetonitrile were used as received. Various alkyl amines were purchased from commercial suppliers and were distilled over potassium hydroxide pellets under air atmosphere. Nitrones were prepared according to literature procedures. ${ }^{1,2}$ The synthesis of ligands $\mathbf{2 a - 2 d}, \mathbf{2 e - 2}, \mathbf{2 m}$, and 2n-2o have been reported. ${ }^{3}$

\section{The Synthesis of Chiral Ligand 2p.}

(S)-2-(dipyridin-2-ylmethyl)-4-isopropyl-4,5-dihydrooxazole 2p<smiles>CC(C)[C@@H]1COC(C(c2ccccn2)c2ccccn2)=N1</smiles>

To a solution of dipyridin-2-ylmethane $(272 \mathrm{mg}, 1.6 \mathrm{mmol})$ in dried THF $(30 \mathrm{~mL})$ was added dropwise $t$-BuLi $(0.95 \mathrm{~mL}, 1.7 \mathrm{M}$ in hexanes, $1.6 \mathrm{mmol})$ within 15-20 minutes at $-78^{\circ} \mathrm{C}$ under $\mathrm{N}_{2}$. The resulting yellow solution was stirred for an additional hour at this temperature. Then a solution of (4S)-2-bromo-4-isopropyl-4, 5-dihydro-oxazole $(357 \mathrm{mg}, 1.9 \mathrm{mmol})$ in THF $(10 \mathrm{~mL})$ was added dropwise at $-78^{\circ} \mathrm{C}$ over 10 minutes. The solution was slowly warmed to room temperature and was stirred for an hour, then refluxed for six hours. The mixture was diluted with $\mathrm{CH}_{2} \mathrm{Cl}_{2}$ (30 mL) and was washed with $\mathrm{H}_{2} \mathrm{O}(5 \mathrm{~mL})$. The aqueous layer was extracted with $\mathrm{CH}_{2} \mathrm{Cl}_{2}(10 \mathrm{~mL})$ and the combined organic phases were dried over $\mathrm{Na}_{2} \mathrm{SO}_{4}$, filtered, and concentrated. The residue was purified by flash chromatography $\left(\mathrm{EtOAc} / \mathrm{Et}_{3} \mathrm{~N}=\right.$ 200/1) to give the desired product (syrup): $257 \mathrm{mg}(57 \%) .[\alpha]^{20}{ }_{\mathrm{D}}-37.0$ (c 0.75 , $\mathrm{CHCl}_{3}$ ); IR (neat) 2961, 2891, 1671, 1586, 1567, 1467, 1432, 1356, 997, $989 \mathrm{~cm}^{-1} ;{ }^{1} \mathrm{H}$ NMR (300 MHz, $\left.\mathrm{CDCl}_{3}\right): \delta$ 8.57-8.54 (m, 2H), 7.64-7.60 (m, 2H), 7.48-7.42 (m, 2H), 7.18-7.15 (m, 2H), $5.46(\mathrm{~s}, 1 \mathrm{H}), 4.30-4.22(\mathrm{~m}, 1 \mathrm{H}), 4.05-3.97(\mathrm{~m}, 2 \mathrm{H}), 1.84-1.76(\mathrm{~m}$, $1 \mathrm{H}), 0.93(\mathrm{~d}, J=6.9 \mathrm{~Hz}, 3 \mathrm{H}), 0.85(\mathrm{~d}, J=6.9 \mathrm{~Hz}, 3 \mathrm{H}) ;{ }^{13} \mathrm{C} \mathrm{NMR}\left(75 \mathrm{MHz}, \mathrm{CDCl}_{3}\right)$ : 865.4, 158.1, 158.0, 149.4, 149.4, 136.6, 123.7, 123.6, 122.2, 122.2, 72.0, 70.0, 56.2, 32.4, 18.9, 17.7; LRMS-EI(m/z): $281\left(\mathrm{M}^{+}\right), 169(100)$; HRMS-ESI $(m / z):[\mathrm{M}+\mathrm{H}]^{+}$ 
calcd for $\mathrm{C}_{17} \mathrm{H}_{19} \mathrm{~N}_{3} \mathrm{O}$, 282.1601; found, 282.1617.

\section{General Procedures for Catalytic Asymmetric Synthesis of $\beta$-Lactams.}

Procedure A: A mixture of $\mathrm{Cu}\left(\mathrm{ClO}_{4}\right)_{2} \cdot 6 \mathrm{H}_{2} \mathrm{O}(9.3 \mathrm{mg}, 0.025 \mathrm{mmol})$ and (S)-isopropyl-trisoxazoline 2a $(11.3 \mathrm{mg}, 0.03 \mathrm{mmol})$ in $\mathrm{CH}_{3} \mathrm{CN}(4 \mathrm{~mL})$ was stirred under air atmosphere at $15{ }^{\circ} \mathrm{C}$ for two hours. The solution was cooled to $0{ }^{\circ} \mathrm{C}$ and then $\mathrm{Cy}_{2} \mathrm{NH}(50 \mu \mathrm{L}, 0.25 \mathrm{mmol})$ was added. After ten minutes, alkyne $(0.375 \mathrm{mmol})$ was added. When the color of the resulting mixture turned into light yellow, to this solution was added nitrone $(0.25 \mathrm{mmol})$. After the reaction was complete (monitored by TLC), the mixture was passed through a short silica gel column (neat $\mathrm{CH}_{2} \mathrm{Cl}_{2}$ as the eluent). The filtrate was concentrated and the residue was purified by flash chromatography $\left(\mathrm{PE} / \mathrm{CH}_{2} \mathrm{Cl}_{2}\right)$ to afford the product. The diasteroselectivity was determinated by ${ }^{1} \mathrm{H}$ NMR spectroscopic analysis of crude product. The determination of enantiomeric excess of cis isomer was performed by chiral HPLC with a Daicel Chiralcel OD-H column (eluent: Hexane/ ${ }^{i} \mathrm{PrOH}(80 / 20)$, flow rate: $0.7 \mathrm{~mL} / \mathrm{min}$ ).

Procedure B: A mixture of $\mathrm{Cu}\left(\mathrm{ClO}_{4}\right)_{2} \cdot 6 \mathrm{H}_{2} \mathrm{O}(4.6 \mathrm{mg}, 0.0125 \mathrm{mmol})$ and (S)- ${ }^{t} \mathrm{Bu}-\mathrm{BOX}$ 1b (4.5 mg, $\left.0.015 \mathrm{mmol}\right)$ in $\mathrm{CH}_{3} \mathrm{CN}(2 \mathrm{~mL})$ was stirred under air atmosphere at $15{ }^{\circ} \mathrm{C}$ for two hours. The solution was cooled to $0{ }^{\circ} \mathrm{C}$ and then ${ }^{i} \operatorname{Pr}_{2} \mathrm{NEt}$ ( $9 \mu \mathrm{L}, 0.05 \mathrm{mmol})$ and nitrone $(0.25 \mathrm{mmol})$ was added. After ten minutes, ethyl propiolate $(50.6 \mu \mathrm{L}, 0.5 \mathrm{mmol})$ was added. After the reaction was complete (monitored by TLC), the mixture was passed through a short silica gel column (neat $\mathrm{CH}_{2} \mathrm{Cl}_{2}$ as the eluent). The filtrate was concentrated and the residue was purified by flash chromatography $\left(\mathrm{PE} / \mathrm{CH}_{2} \mathrm{Cl}_{2}\right)$ to afford the product. The diasteroselectivity was

determined by ${ }^{1} \mathrm{H}$ NMR spectroscopic analysis of crude product. The determination of enantiomeric excess of trans isomer was performed by chiral HPLC with a Daicel Chiralcel OD-H column (eluent: Hexane/ ${ }^{i} \mathrm{PrOH}(85 / 15)$, flow rate: $\left.0.9 \mathrm{~mL} / \mathrm{min}\right)$.

(3S,4S)-1,3,4-triphenyl-2-azetidinone $6 a^{5}$ 


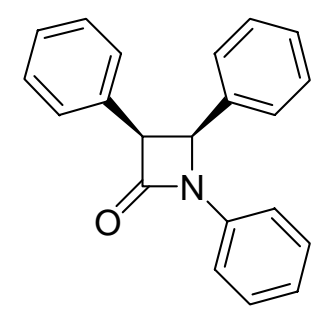

Procedure A: $56 \%$ yield (solid, $35 \mathrm{~h}$ ); $[\alpha]^{20}{ }_{\mathrm{D}}-8.0$ (c 1.62, $\mathrm{CHCl}_{3} ; 82 \%$ ee); ${ }^{1} \mathrm{H}$ NMR $\left(300 \mathrm{MHz}, \mathrm{CDCl}_{3}\right): \delta$ 7.43-7.40 (m, 2H), 7.29-7.24 (m, 2H), 7.09-7.03 (m, 11H), 5.45 $(\mathrm{d}, J=6.3 \mathrm{~Hz}, 1 \mathrm{H}), 5.00(\mathrm{~d}, J=6.6 \mathrm{~Hz}, 1 \mathrm{H})$.

\section{(3S,4S)-1-(4-Methylphenyl)-3,4-diphenyl-2-azetidinone $6 b^{6}$}

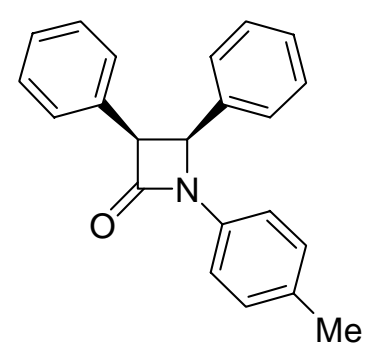

Procedure A: $36 \%$ yield (solid, $56 \mathrm{~h}$ ); $[\alpha]^{20}{ }_{\mathrm{D}}-10.5$ (c $0.68, \mathrm{CHCl}_{3} ; 82 \%$ ee); ${ }^{1} \mathrm{H} \mathrm{NMR}$ $\left(300 \mathrm{MHz}, \mathrm{CDCl}_{3}\right): \delta 7.32-7.26(\mathrm{~m}, 2 \mathrm{H}), 7.11-7.03(\mathrm{~m}, 12 \mathrm{H}), 5.44(\mathrm{~d}, J=6.0 \mathrm{~Hz}, 1 \mathrm{H})$, $5.00(\mathrm{~d}, J=5.7 \mathrm{~Hz}, 1 \mathrm{H}), 2.29(\mathrm{~s}, 3 \mathrm{H})$.

(3S,4S)-1-(4-Methoxylphenyl)-3,4-diphenyl-2-azetidinone $6 c^{7}$

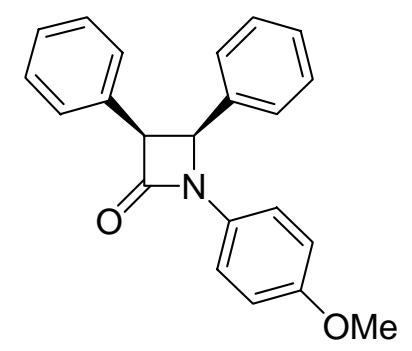

Procedure A: $36 \%$ yield (solid, 7 days); $[\alpha]^{20}{ }_{\mathrm{D}}-4.8$ (c $0.63, \mathrm{CHCl}_{3} ; 84 \%$ ee); ${ }^{1} \mathrm{H}$ NMR (300 MHz, $\left.\mathrm{CDCl}_{3}\right): \delta 7.36(\mathrm{dd}, J=2.1,6.9 \mathrm{~Hz}, 2 \mathrm{H}), 7.11-7.03(\mathrm{~m}, 10 \mathrm{H}), 6.82$ $(\mathrm{dd}, J=2.1,6.6 \mathrm{~Hz}, 2 \mathrm{H}), 5.42(\mathrm{~d}, J=6.0 \mathrm{~Hz}, 1 \mathrm{H}), 5.00(\mathrm{~d}, J=6.3 \mathrm{~Hz}, 1 \mathrm{H}), 3.76(\mathrm{~s}$, $3 \mathrm{H})$.

(3S,4S)-1-(4-Bromophenyl)-3,4-diphenyl-2-azetidinone $6 \mathrm{~d}^{1}$ 


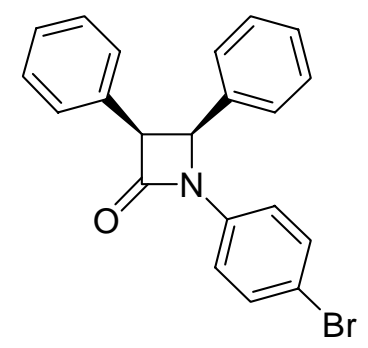

Procedure A: 70\% yield (solid, $33 \mathrm{~h}$ ); $[\alpha]^{20}{ }_{\mathrm{D}}-16\left(\mathrm{c} 0.29, \mathrm{CHCl}_{3} ; 75 \%\right.$ ee); ${ }^{1} \mathrm{H}$ NMR $\left(300 \mathrm{MHz}, \mathrm{CDCl}_{3}\right): \delta 7.39(\mathrm{~d}, J=8.7 \mathrm{~Hz}, 2 \mathrm{H}), 7.30(\mathrm{~d}, J=9.0 \mathrm{~Hz}, 2 \mathrm{H}), 7.12-7.02(\mathrm{~m}$, $10 \mathrm{H}), 5.45(\mathrm{~d}, J=6.3 \mathrm{~Hz}, 1 \mathrm{H}), 5.03(\mathrm{~d}, J=6.6 \mathrm{~Hz}, 1 \mathrm{H})$.

(3S,4S)-1-(4-Carboethoxyphenyl)-3,4-diphenyl-2-azetidinone $6 \mathrm{e}^{1}$

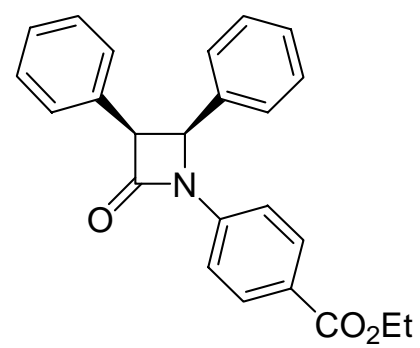

Procedure A: 98\% yield (syrup, 9 h); $[\alpha]^{20}{ }_{D}-11.4$ (c 1.43, $\mathrm{CHCl}_{3} ; 70 \%$ ee); ${ }^{1} \mathrm{H}$ NMR $\left(300 \mathrm{MHz}, \mathrm{CDCl}_{3}\right): \delta 7.98(\mathrm{~d}, J=8.7 \mathrm{~Hz}, 2 \mathrm{H}), 7.44(\mathrm{~d}, J=9.0 \mathrm{~Hz}, 2 \mathrm{H}), 7.12-7.03(\mathrm{~m}$, $10 \mathrm{H}), 5.51(\mathrm{~d}, J=6.6 \mathrm{~Hz}, 1 \mathrm{H}), 5.06(\mathrm{~d}, J=6.3 \mathrm{~Hz}, 1 \mathrm{H}), 4.34(\mathrm{q}, J=6.9 \mathrm{~Hz}, 2 \mathrm{H}), 1.36$ (t, $J=6.9 \mathrm{~Hz}, 3 \mathrm{H})$.

(3S,4S)-4-(4-Methylphenyl)-1,3-diphenyl-2-azetidinone $6 f^{6}$

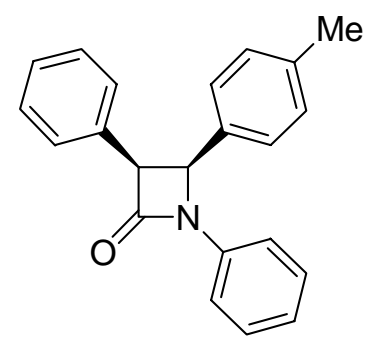

Procedure A: 50\% yield (solid, $56 \mathrm{~h}$ ); $[\alpha]^{20}{ }_{\mathrm{D}}-14.2$ (c $0.95, \mathrm{CHCl}_{3} ; 82 \%$ ee); ${ }^{1} \mathrm{H}$ NMR $\left(300 \mathrm{MHz}, \mathrm{CDCl}_{3}\right) \delta$ 7.43-7.39 (m, 2H), 7.30-7.25 (m, 2H), 7.10-7.05 (m, 6H), 6.97-6.90 (m, 4H), $5.44(\mathrm{~d}, J=6.0 \mathrm{~Hz}, 1 \mathrm{H}), 4.98(\mathrm{~d}, J=6.0 \mathrm{~Hz}, 1 \mathrm{H}), 2.18(\mathrm{~s}, 3 \mathrm{H})$.

(3S,4S)-4-(4-Methoxylphenyl)-1,3-diphenyl-2-azetidinone $6 g^{8}$ 


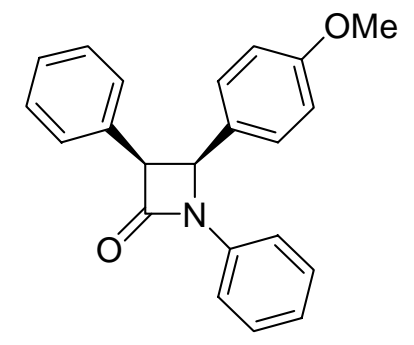

Procedure A: $58 \%$ yield (solid, 8 days); $[\alpha]^{20}-11.6$ (c $0.98, \mathrm{CHCl}_{3} ; 83 \%$ ee); ${ }^{1} \mathrm{H}$ NMR (300 MHz, $\left.\mathrm{CDCl}_{3}\right) \delta$ 7.43-7.40 (m, 2H), 7.31-7.25 (m, 2H), 7.12-7.07 (m, 6H), $7.00(\mathrm{dd}, J=2.4,6.9 \mathrm{~Hz}, 2 \mathrm{H}), 6.65(\mathrm{dd}, J=2.1,6.6 \mathrm{~Hz}, 2 \mathrm{H}), 5.43(\mathrm{~d}, J=6.0 \mathrm{~Hz}, 1 \mathrm{H})$, $4.99(\mathrm{~d}, J=6.3 \mathrm{~Hz}, 1 \mathrm{H}), 3.67$ (s, 3H).

(3S,4S)-4-(4-Trifluoromethylphenyl)-1,3-diphenyl-2-azetidinone $6 \mathrm{~h}$

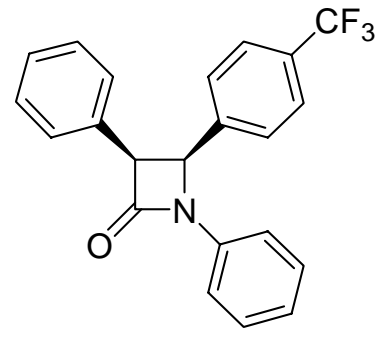

Procedure A: $75 \%$ yield (solid, 22 hours); $[\alpha]^{20}{ }_{\mathrm{D}}-6.6\left(\mathrm{c} 0.89, \mathrm{CHCl}_{3} ; 82 \%\right.$ ee); ${ }^{1} \mathrm{H}$ NMR (300 MHz, $\left.\mathrm{CDCl}_{3}\right): \delta$ 7.40-7.26 (m, 6H), $7.18(\mathrm{~d}, J=8.4 \mathrm{~Hz}, 2 \mathrm{H}), 7.13-7.01(\mathrm{~m}$, $6 \mathrm{H}), 5.51(\mathrm{~d}, J=6.0 \mathrm{~Hz}, 1 \mathrm{H}), 5.06(\mathrm{~d}, J=6.0 \mathrm{~Hz}, 1 \mathrm{H}) ;{ }^{13} \mathrm{C} \mathrm{NMR}\left(75 \mathrm{MHz}, \mathrm{CDCl}_{3}\right): \delta$ $165.2,142.3,138.7,137.3,131.4,130.0\left(\mathrm{q},{ }^{2} J_{\mathrm{C}-\mathrm{F}}=40\right), 129.2,128.7,128.3,127.6$, $127.4,125.2\left(\mathrm{q},{ }^{3} J_{\mathrm{C}-\mathrm{F}}=3.8\right), 124.4,123.7\left(\mathrm{q},{ }^{1} J_{\mathrm{C}-\mathrm{F}}=256\right), 117.1,60.4,59.7 ; \mathrm{IR}(\mathrm{KBr})$ : 3033, 2925, 1755, 1621, 1599, 1498, 1454, 1422, 1379, 1326, 1168, 1125, 1069, 1018, 885, 837, 754, $698 \mathrm{~cm}^{-1}$; EI (m/z): $367\left(\mathrm{M}^{+}\right)$(10), 248 (100); Anal. Calcd. For $\mathrm{C}_{22} \mathrm{H}_{16} \mathrm{~F}_{3} \mathrm{NO}$ : C, 71.93; H, 4.39; N, 3.81. Found: C, 71.71; H, 4.44; N, 3.65.

(3S,4S)-4-(2-furyl)-1,3-diphenyl-2-azetidinone $6 i^{9}$<smiles>O=C1[C@H](c2ccccc2)[C@H](c2ccco2)N1c1ccccc1</smiles> 
Procedure A: $56 \%$ yield (solid, 7 days); $[\alpha]^{20}{ }_{\mathrm{D}}-37.4$ (c $0.66, \mathrm{CHCl}_{3} ; 85 \%$ ee); ${ }^{1} \mathrm{H}$ NMR (300 MHz, $\left.\mathrm{CDCl}_{3}\right): \delta$ 7.45-7.41 (m, 2H), 7.33-7.26 (m, 2H), 7.22-7.19 (m, 5H), 7.13-7.09 (m, 2H), 6.12-6.11 (m, 2H), $5.47(\mathrm{~d}, J=5.7 \mathrm{~Hz}, 1 \mathrm{H}), 4.97(\mathrm{~d}, J=6.0 \mathrm{~Hz}$, $1 \mathrm{H})$.

(3S,4S)-1-(4-Methoxylphenyl)-4-(4-trifluoromethylphenyl)-3-phenyl-2-azetidinoe $\mathbf{6 j}^{1}$

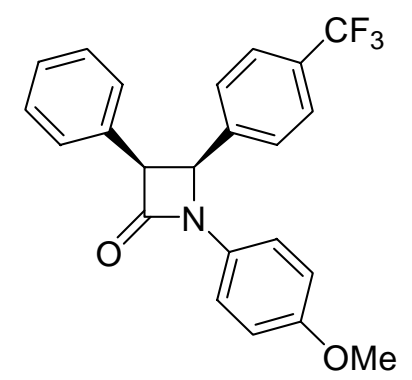

Procedure A: 35\% yield (solid, 7 days); $[\alpha]^{20}-4.0$ (c $0.80, \mathrm{CHCl}_{3} ; 84 \%$ ee); ${ }^{1} \mathrm{H}$ NMR (300 MHz, $\left.\mathrm{CDCl}_{3}\right): \delta 7.36(\mathrm{~d}, J=8.4 \mathrm{~Hz}, 2 \mathrm{H}), 7.32$ (d, $\left.J=9.0 \mathrm{~Hz}, 2 \mathrm{H}\right), 7.17$ (d, $J=8.1 \mathrm{~Hz}, 2 \mathrm{H}), 7.09-7.03(\mathrm{~m}, 5 \mathrm{H}), 6.83(\mathrm{~d}, J=8.7 \mathrm{~Hz}, 2 \mathrm{H}), 5.47 \mathrm{~d}, J=5.7 \mathrm{~Hz}, 1 \mathrm{H})$, $5.04(\mathrm{~d}, J=6.0 \mathrm{~Hz}, 1 \mathrm{H}), 3.76(\mathrm{~s}, 3 \mathrm{H})$.

(3S,4S)-3-(4-Trifluoromethylphenyl)-1,4-diphenyl-2-azetidinone 6k

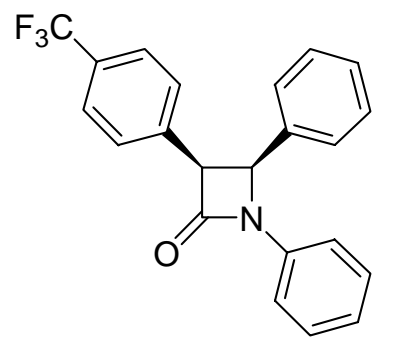

Procedure A: 65\% yield (solid, $40 \mathrm{~h}$ ); $[\alpha]^{20}{ }_{\mathrm{D}}-6.5$ (c $0.63, \mathrm{CHCl}_{3} ; 73 \%$ ee); ${ }^{1} \mathrm{H} \mathrm{NMR}$ $\left(300 \mathrm{MHz}, \mathrm{CDCl}_{3}\right): \delta 7.42-7.30(\mathrm{~m}, 6 \mathrm{H}), 7.20-7.05(\mathrm{~m}, 8 \mathrm{H}), 5.51(\mathrm{~d}, J=6.0 \mathrm{~Hz}, 1 \mathrm{H})$, $5.04(\mathrm{~d}, J=6.0 \mathrm{~Hz}, 1 \mathrm{H}) ;{ }^{13} \mathrm{C} \mathrm{NMR}\left(75 \mathrm{MHz}, \mathrm{CDCl}_{3}\right): \delta 164.6,137.4,136.2,133.8$, $129.9\left(\mathrm{q},{ }^{2} J_{\mathrm{C}-\mathrm{F}}=40\right), 129.1,128.5,128.3,126.9,125.0\left(\mathrm{q},{ }^{3} J_{\mathrm{C}-\mathrm{F}}=3.6\right), 124.3,123.9(\mathrm{q}$, $\left.{ }^{1} J_{\mathrm{C}-\mathrm{F}}=256\right), 117.2,60.0,59.6$; IR (KBr): 3038, 2924, 1749, 1736, 1599, 1500, 1382 , 1322, 1167, 1125, 1069, 885, 819, 763, $702 \mathrm{~cm}^{-1}$; EI (m/z): $367\left(\mathrm{M}^{+}\right)(8), 181(100)$; Anal. Calcd. For $\mathrm{C}_{22} \mathrm{H}_{16} \mathrm{~F}_{3} \mathrm{NO}$ : C, 71.93; H, 4.39; N, 3.81. Found: C, 71.71; H, 4.13; 
N, 3.52.

(3S,4S)-3-(Cyclohex-1-enyl)-1,4-diphenyl-2-azetidinone $6 l^{8}$

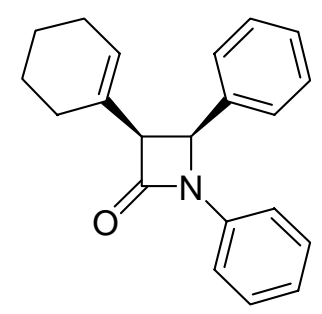

Procedure A: $33 \%$ yield (solid, 5 days); $[\alpha]^{20}{ }_{\mathrm{D}}-10.8$ (c $0.72, \mathrm{CHCl}_{3} ; 72 \%$ ee); ${ }^{1} \mathrm{H}$ NMR (300 MHz, $\left.\mathrm{CDCl}_{3}\right): \delta$ 7.36-7.21 (m, 9H), 7.06-7.01 (m, 1H), 5.86-5.85 (m, 1H), $5.22(\mathrm{~d}, J=5.7 \mathrm{~Hz}, 1 \mathrm{H}), 4.18(\mathrm{~d}, J=6.0 \mathrm{~Hz}, 1 \mathrm{H}), 2.12-0.85(\mathrm{~m}, 8 \mathrm{H})$.

(3R,4S)-ethyl 2-oxo-1,4-diphenylazetidine-3-carboxylate $6 m^{10}$

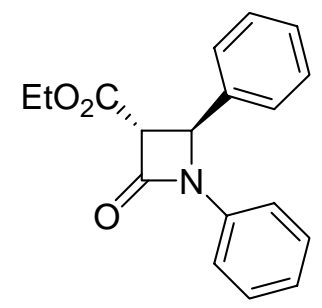

Procedure B: $67 \%$ yield (syrup, $1 \mathrm{~h}$ ); $[\alpha]^{20}{ }_{\mathrm{D}} 34.9$ (c 0.84, $\mathrm{CHCl}_{3} ; 50 \%$ ee); ${ }^{1} \mathrm{H}$ NMR (300 MHz, $\left.\mathrm{CDCl}_{3}\right): \delta$ 7.41-7.23 (m, 9H), 7.09-7.07 (m, 1H), $5.35(\mathrm{~d}, J=3.0 \mathrm{~Hz}, 1 \mathrm{H})$, $4.30(\mathrm{q}, J=6.9 \mathrm{~Hz}, 2 \mathrm{H}), 3.99(\mathrm{~d}, J=2.7 \mathrm{~Hz}, 1 \mathrm{H}), 1.33(\mathrm{t}, J=7.2 \mathrm{~Hz}, 8 \mathrm{H}) ;{ }^{13} \mathrm{C} \mathrm{NMR}$ $\left(75 \mathrm{MHz}, \mathrm{CDCl}_{3}\right): \delta 166.1,159.1,137.0,136.2,129.2,129.0,128.9,126.0,124.3$, $117.0,63.4,62.0,57.4,14.1$.

(3R,4S)-ethyl

1-(4-(ethoxycarbonyl)phenyl)-2-oxo-4-phenylazetidine-3-carboxylate 6n

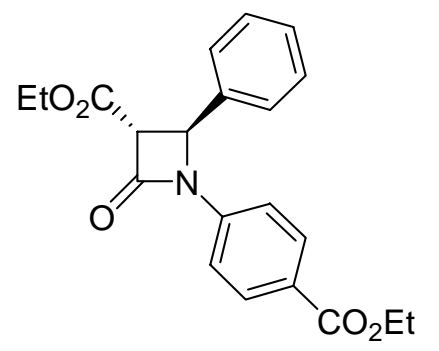

Procedure B: $80 \%$ yield (syrup, $1 \mathrm{~h}$ ); $[\alpha]^{20}{ }_{\mathrm{D}} 17.4$ (c $0.88, \mathrm{CHCl}_{3} ; 49 \%$ ee); ${ }^{1} \mathrm{H} \mathrm{NMR}$ 
(300 MHz, $\left.\mathrm{CDCl}_{3}\right): \delta 7.96-7.93(\mathrm{~m}, 2 \mathrm{H}), 7.40-7.27(\mathrm{~m}, 7 \mathrm{H}), 5.38(\mathrm{~d}, J=2.4 \mathrm{~Hz}, 1 \mathrm{H})$, $4.33(\mathrm{q}, J=7.2 \mathrm{~Hz}, 2 \mathrm{H}), 4.30(\mathrm{q}, J=7.2 \mathrm{~Hz}, 2 \mathrm{H}), 4.03$ (d, $J=2.7 \mathrm{~Hz}, 1 \mathrm{H}), 1.35$ (t, $J=$ $7.2 \mathrm{~Hz}, 3 \mathrm{H}), 1.34(\mathrm{t}, J=7.2 \mathrm{~Hz}, 3 \mathrm{H}) ;{ }^{13} \mathrm{C} \mathrm{NMR}\left(75 \mathrm{MHz}, \mathrm{CDCl}_{3}\right): \delta 165.9,165.8$, $159.5,140.5,135.7,130.8,129.4,129.2,126.1,126.0,116.6,36.7,62.2,60.9,57.7$, 14.2, 14.1; IR (KBr): 2982, 2938, 1768, 1723, 1606, 1514, 1456, 1365, 1173, 1114, 1013, 854, $769 \mathrm{~cm}^{-1}$; EI (m/z): $367\left(\mathrm{M}^{+}\right), 131$ (100); Anal. Calcd. For $\mathrm{C}_{22} \mathrm{H}_{16} \mathrm{~F}_{3} \mathrm{NO}: \mathrm{C}$, 68.31; H, 5.76; N, 3.81. Found: C, 68.31; H, 5.85; N, 3.46.

\section{(3R,4S)-ethyl 1-phenyl-2-oxo-4-(2-furyl)azetidine-3-carboxylate 60}

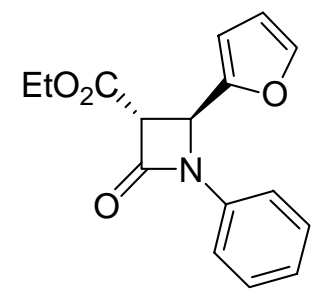

Procedure B: 78\% yield (syrup, $2 \mathrm{~h}$ ); $[\alpha]^{20}{ }_{\mathrm{D}} 13.9$ (c 1.92, $\mathrm{CHCl}_{3} ; 45 \%$ ee); ${ }^{1} \mathrm{H} \mathrm{NMR}$ $\left(300 \mathrm{MHz}, \mathrm{CDCl}_{3}\right): \delta$ 7.42-7.25 (m, 5H), 7.11-7.06 (m, 1H), $6.54(\mathrm{~d}, J=3.6 \mathrm{~Hz}, 1 \mathrm{H})$, $6.38(\mathrm{dd}, J=2.1,3.3 \mathrm{~Hz}, 1 \mathrm{H}), 5.38(\mathrm{~d}, J=2.4 \mathrm{~Hz}, 1 \mathrm{H}), 4.33(\mathrm{~d}, J=2.7 \mathrm{~Hz}, 1 \mathrm{H}), 4.28$ $(\mathrm{q}, J=7.2 \mathrm{~Hz}, 2 \mathrm{H}), 1.33(\mathrm{t}, J=7.5 \mathrm{~Hz}, 3 \mathrm{H}) ;{ }^{13} \mathrm{C} \mathrm{NMR}\left(75 \mathrm{MHz}, \mathrm{CDCl}_{3}\right): \delta 166.0$, 158.9, 148.4, 143.6, 137.2, 129.0, 124.5, 116.8, 110.7, 110.6, 62.2, 60.2, 50.7, 14.1; IR (KBr): 2985, 2356, 1769, 1727, 1590, 1545, 1497, 1369, 1294, 1154, 1010, 752 $\mathrm{cm}^{-1}$; EI (m/z): $285\left(\mathrm{M}^{+}\right), 166(100)$; HRMS-ESI $(\mathrm{m} / \mathrm{z}):[\mathrm{M}+\mathrm{Na}]^{+}$calcd for $\mathrm{C}_{16} \mathrm{H}_{15} \mathrm{NO}_{4}$, 308.0899; found, 308.0891 .

Absolute configurations of $\beta$-lactams 6a-6l were determined by comparison of their optical rotations with those of the known compounds. For $\beta$-Lactam 6o, its configuration was determined by chemical transformation into a known compound $7 .{ }^{4}$ (S)-4-(furan-2-yl)-1-phenyl-2-azetidinone $7^{4}$ 


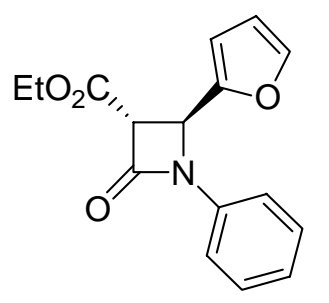

60

\section{1. $\mathrm{KOH}$ (1.0 equiv.) 1,4-dioxane/ $\mathrm{H}_{2} \mathrm{O}(2 / 1)$ \\ 2. $\mathrm{Et}_{3} \mathrm{~N}$, reflux}

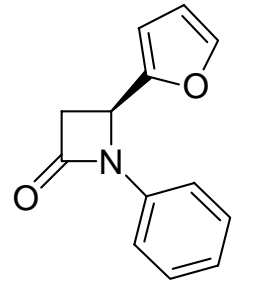

7

To a solution of compound 60 ( $45 \%$ ee, $143 \mathrm{mg}, 0.5 \mathrm{mmol})$ in 1,4-dioxane (4 mL) and $\mathrm{H}_{2} \mathrm{O}(2 \mathrm{~mL})$ was added $\mathrm{KOH}(56 \mathrm{mg}, 1.0 \mathrm{mmol})$ at room temperature. The resulting mixture was stirred overnight. Then the mixture was washed with $\mathrm{CH}_{2} \mathrm{Cl}_{2}(3 \times 6 \mathrm{~mL})$. The aqueous layer was acidified with $\mathrm{HCl}(1 \mathrm{~N}, 2 \mathrm{~mL})$, extracted with $\mathrm{CH}_{2} \mathrm{Cl}_{2}(3 \times 10$ $\mathrm{mL}$ ), and the combined organic phases were dried over $\mathrm{Na}_{2} \mathrm{SO}_{4}$, filtered, and concentrated to give the crude hydrolysate. Triethylamine $(10 \mathrm{~mL})$ was added and the mixture was refluxed for 10 hours. Cooled to rt, the mixture was concentrated in vacuo, washed with $\mathrm{KOH}(1 \mathrm{~N}, 2 \mathrm{~mL})$, and extracted with $\mathrm{CH}_{2} \mathrm{Cl}_{2}(3 \times 10 \mathrm{~mL})$. The combined organic phases were concentrated and the residue was purified by flash chromatography $\left(\mathrm{CH}_{2} \mathrm{Cl}_{2}\right)$ to give the desired product 7 (solid, 20\% yield). $[\alpha]^{20}$ -82.0 (c 0.42, $\left.\mathrm{CHCl}_{3}\right) ;{ }^{1} \mathrm{H}$ NMR (300 MHz, $\mathrm{CDCl}_{3}$ ): $\delta$ 7.40-7.35 (m, 3H), 7.27-7.24 (m, 2H), $7.05(\mathrm{t}, J=7.5 \mathrm{~Hz}, 1 \mathrm{H}), 6.45(\mathrm{~d}, J=2.7 \mathrm{~Hz}, 1 \mathrm{H}), 6.37-6.35(\mathrm{~m}, 1 \mathrm{H}), 5.06$ $(\mathrm{dd}, J=2.7,5.4 \mathrm{~Hz}, 1 \mathrm{H}), 3.47$ (dd, $J=6.0,15.6 \mathrm{~Hz}, 1 \mathrm{H}), 3.27$ (dd, $J=2.4,15.0 \mathrm{~Hz}$, $1 \mathrm{H})$.

Reference:

1. Lo, M. M.-C.; Fu, G. C. J. Am. Chem. Soc. 2002, 124, 4572.

2. West, P. R.; Davis, G. C. J. Org. Chem. 1989, 54, 5176.

3. (a) Ye, M.-C.; Li, B.; Zhou, J.; Tang, Y. J. Org. Chem. 2005, 70, 6108. (b) Zhou, J.; Ye, M.-C.; Tang, Y. J. Comb. Chem. 2004, 6, 301.

4. (a) Basak, A.; Hahato, T.; Bhattacharya, G.; Mukherjee, B. Tetrahedron Lett. 1997, 38, 643. (b) Baldoli, C.; Buttero, P. D.; Licandro, E.; Antonio Papagni, A.; Pilati, T. Tetrahedron 1996, 52, 4849.

5. Cho, C. S.; Jiang, L. H.; Shim, S. C. Synth. Commun. 1999, 29, 2695.

6. Ding, L. K.; Irwin, W. J. J. Chem. Soc. Perkin Trans. 1 1976, 2382.

7. Hinz, W.; Just, G.; Sacripante, G. Magn. Reson. Chem. 1987, 25, 274. 
8. Miura, M.; Enna, M.; Okuro, K.; Nomura, M. J. Org. Chem. 1995, 60, 4999.

9. Dutta, D. K.; Boruah, R. C.; Sandhu, J. S. Indian J. Chem. Sect. B. 1986, 25, 350.

10. Brown, D. S.; Elliott, M. C.; Moody, C. J.; Mowlem, T. J.; Marino, J. P.; Padwa, A. J. Org. Chem. 1994, 59, 2447. 


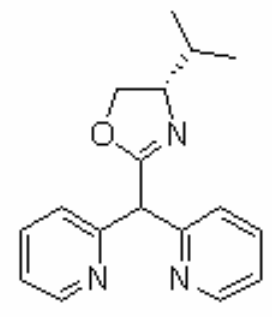

y5-72-4

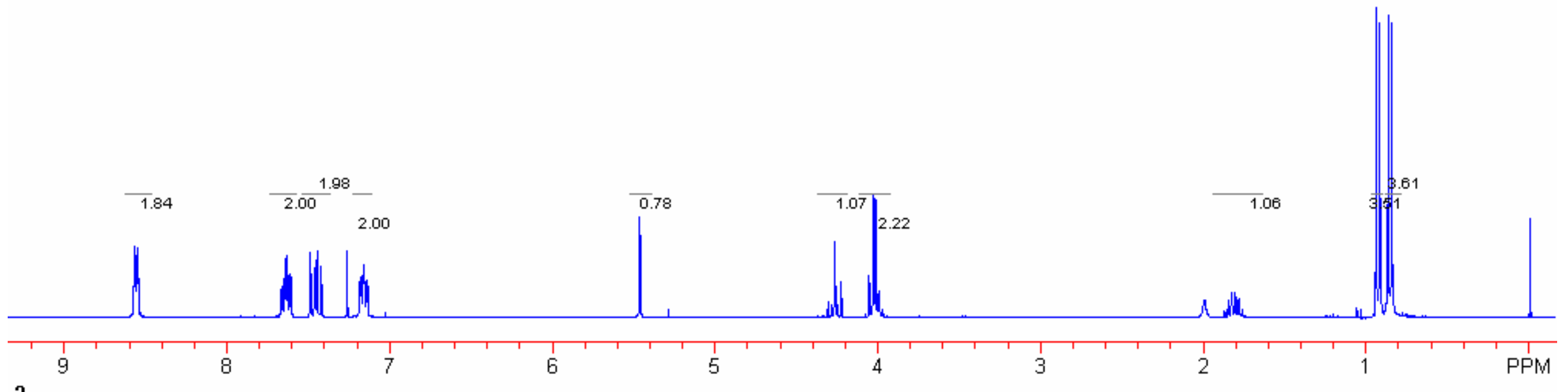



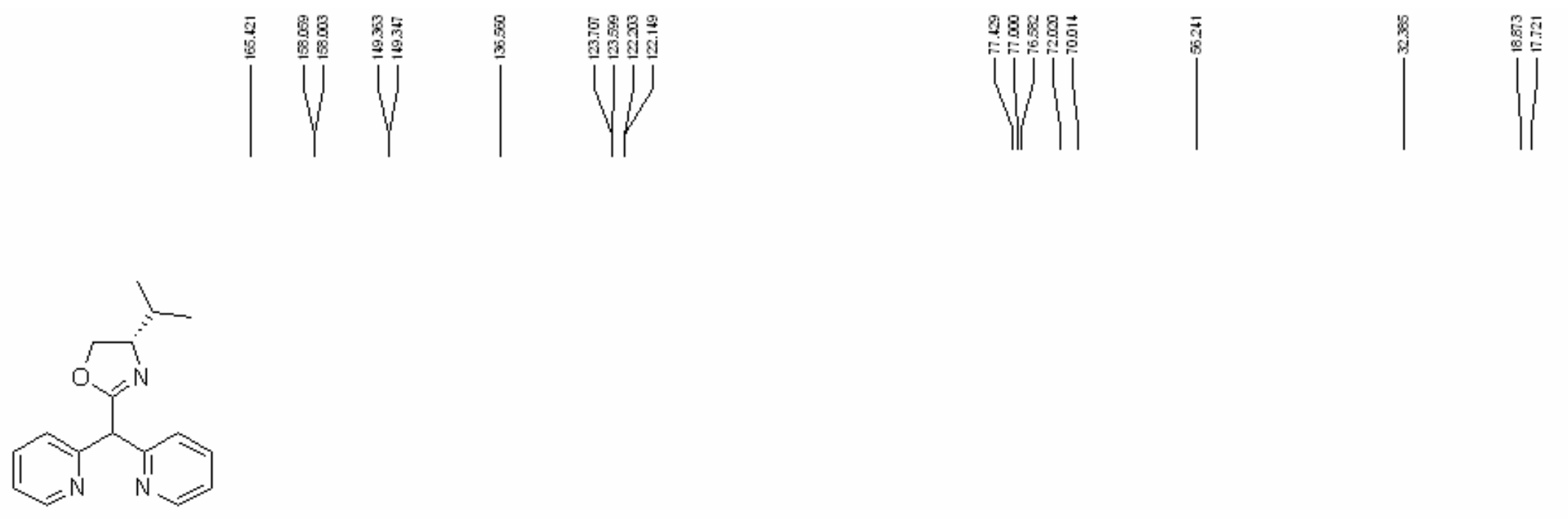

$y 5-72-4$

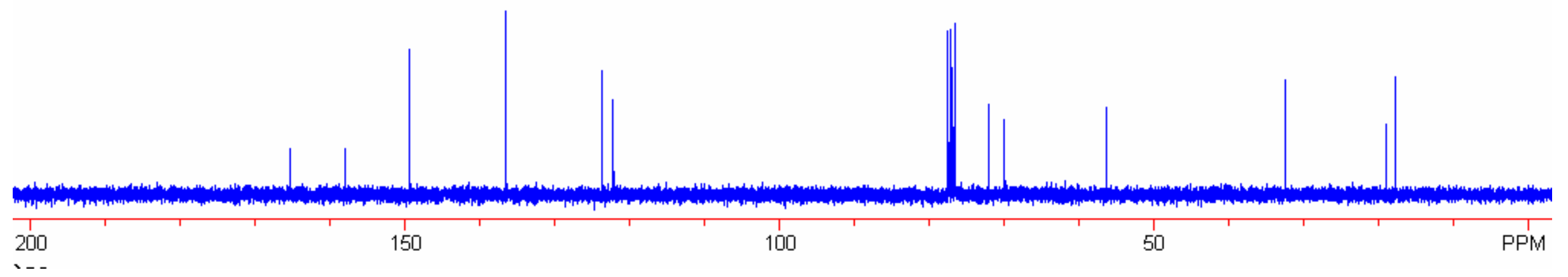




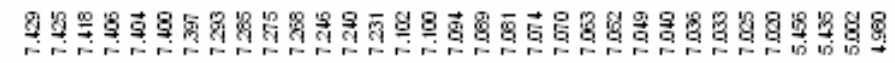

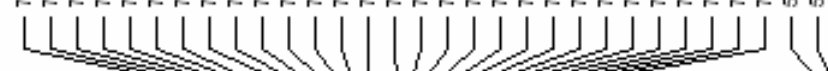
II

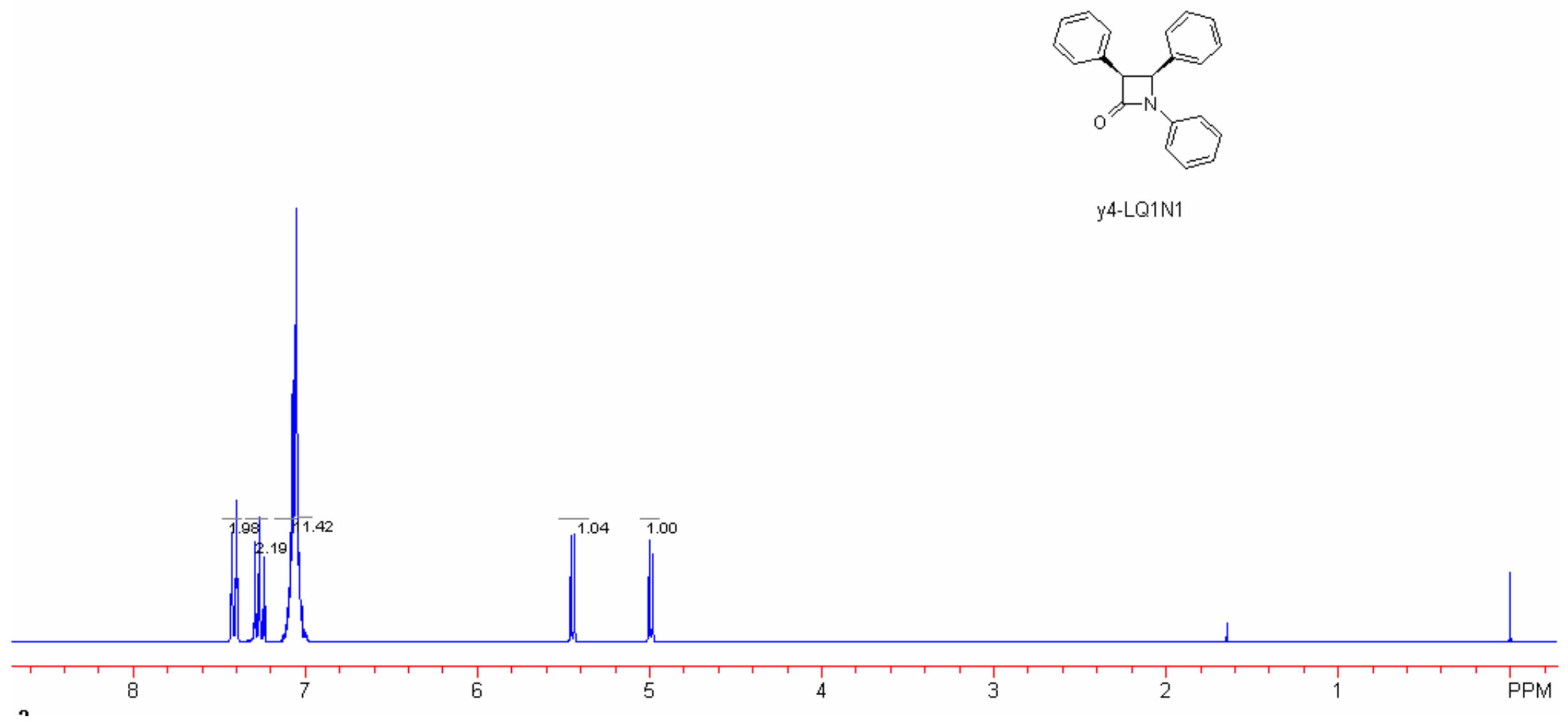



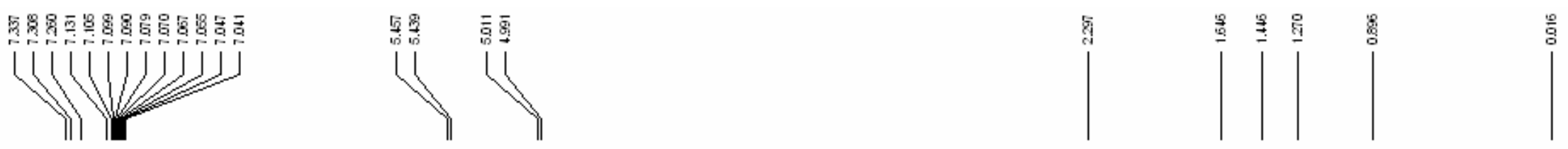<smiles>Cc1ccc(N2C(=O)[C@@H](c3ccccc3)[C@H]2c2ccccc2)cc1</smiles>

Lactam-N-Me

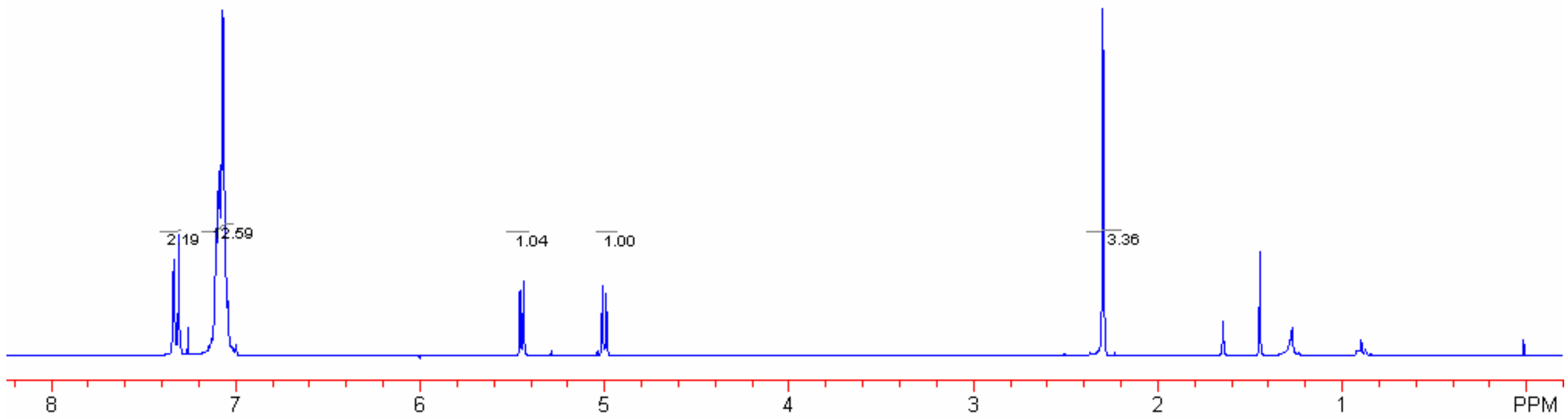

S15 

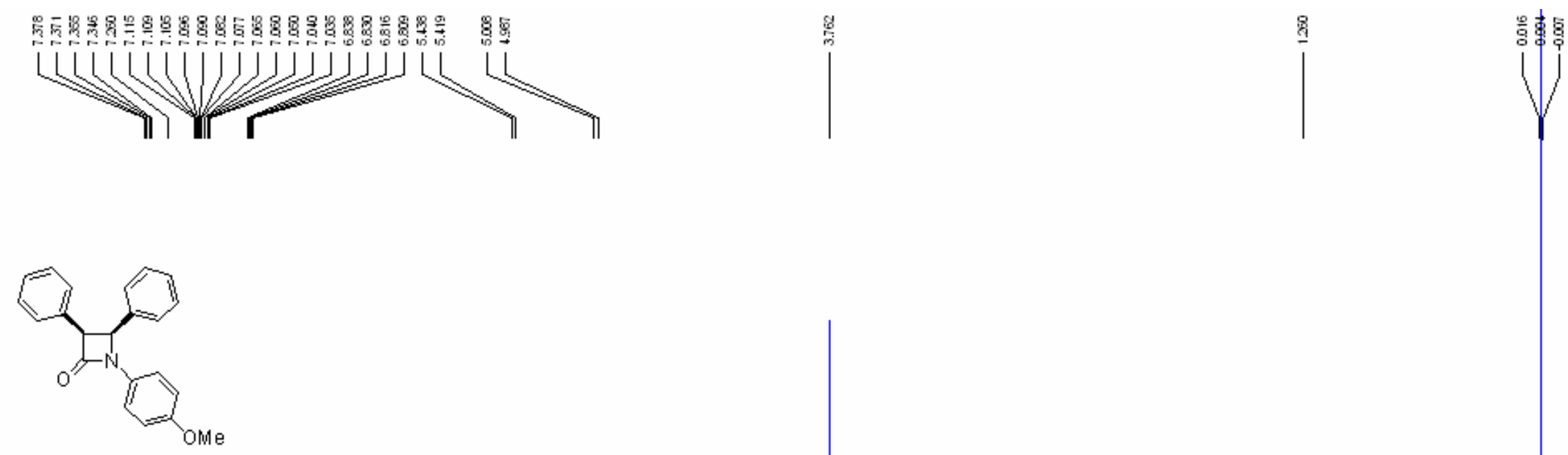

$y$-lactamQ1N2

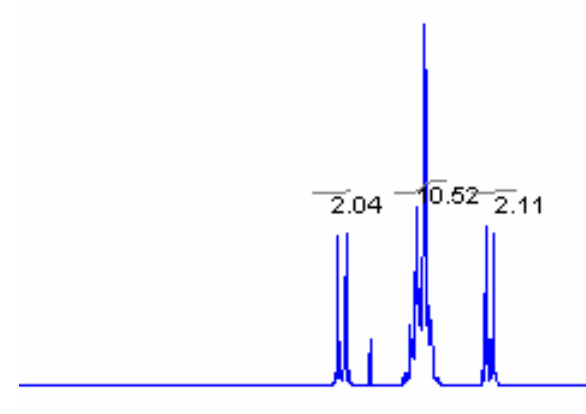

8

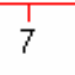

5

3

2

PPM 

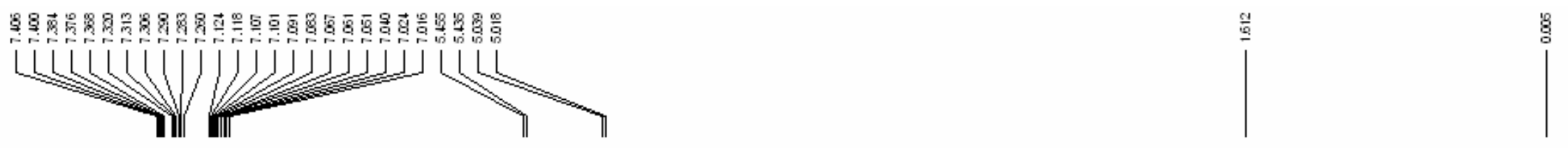

Lactam-Q1NBr<smiles>O=C1[C@H](c2ccccc2)C(c2ccccc2)N1c1ccc(Br)cc1</smiles>

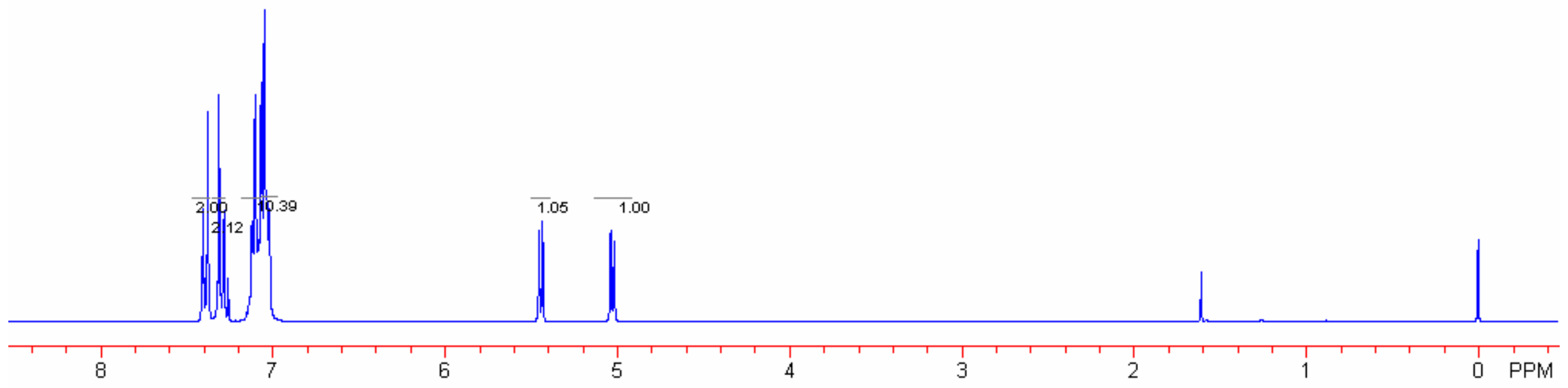

S17 


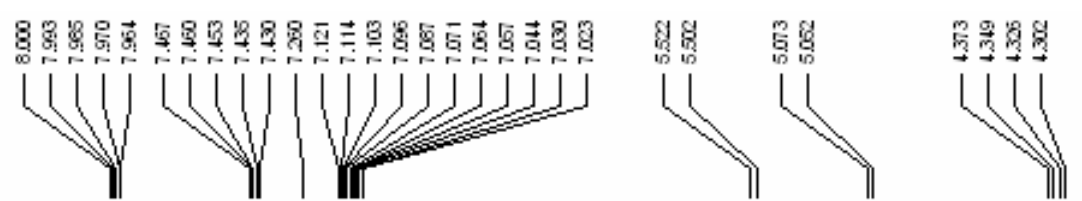

88

$y-\operatorname{lq} 1 N 3$

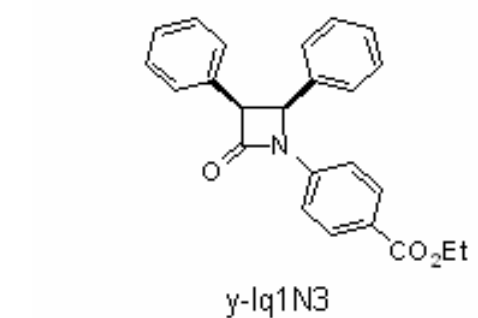

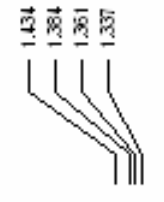

g

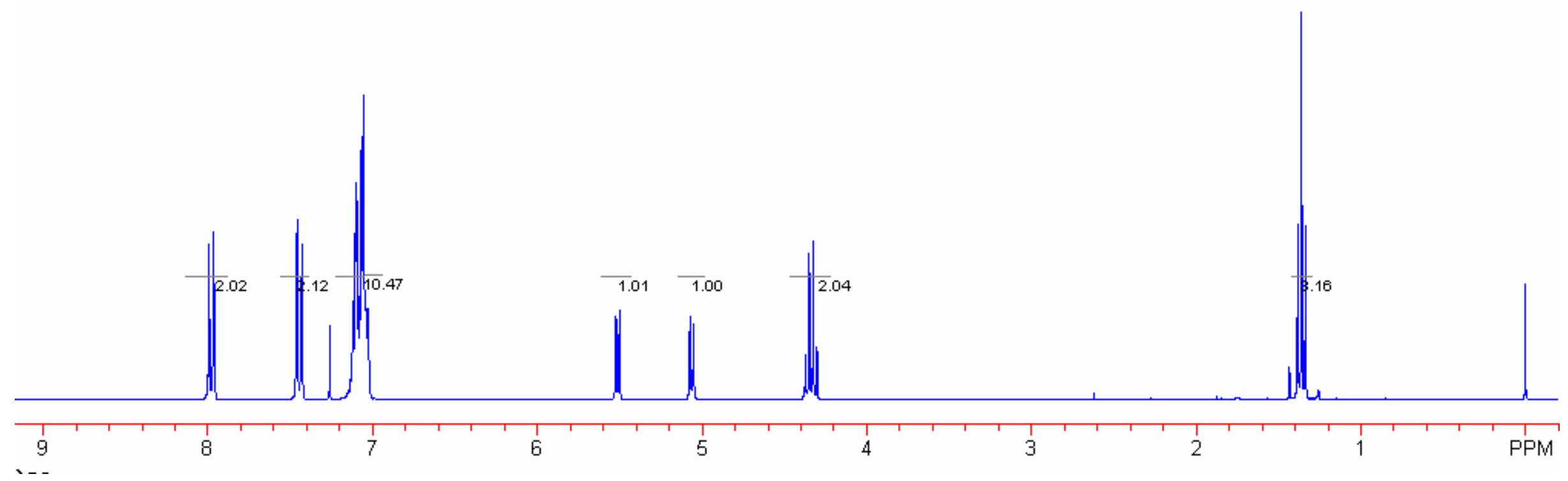




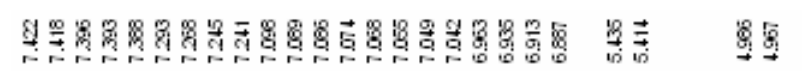
i|i่)
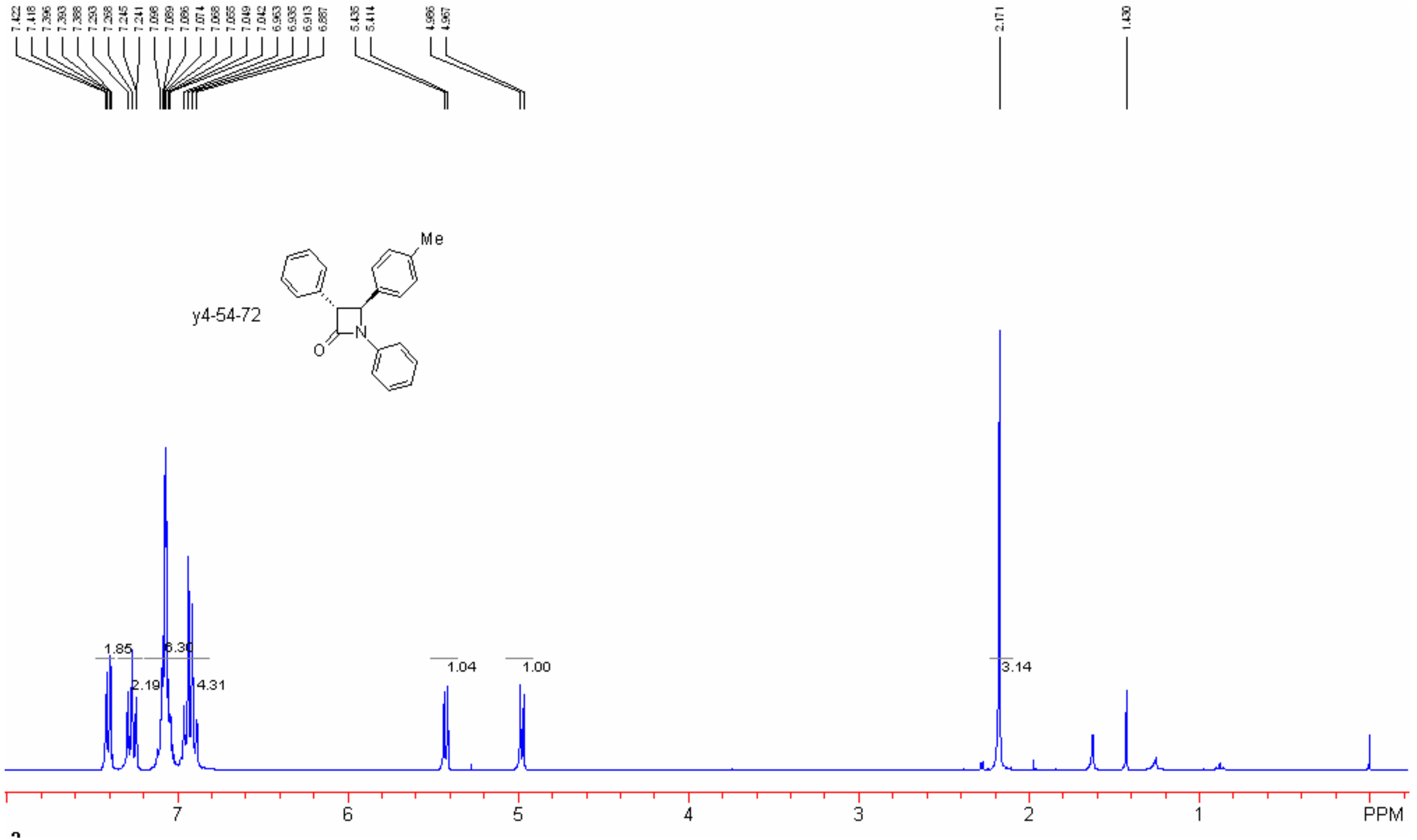

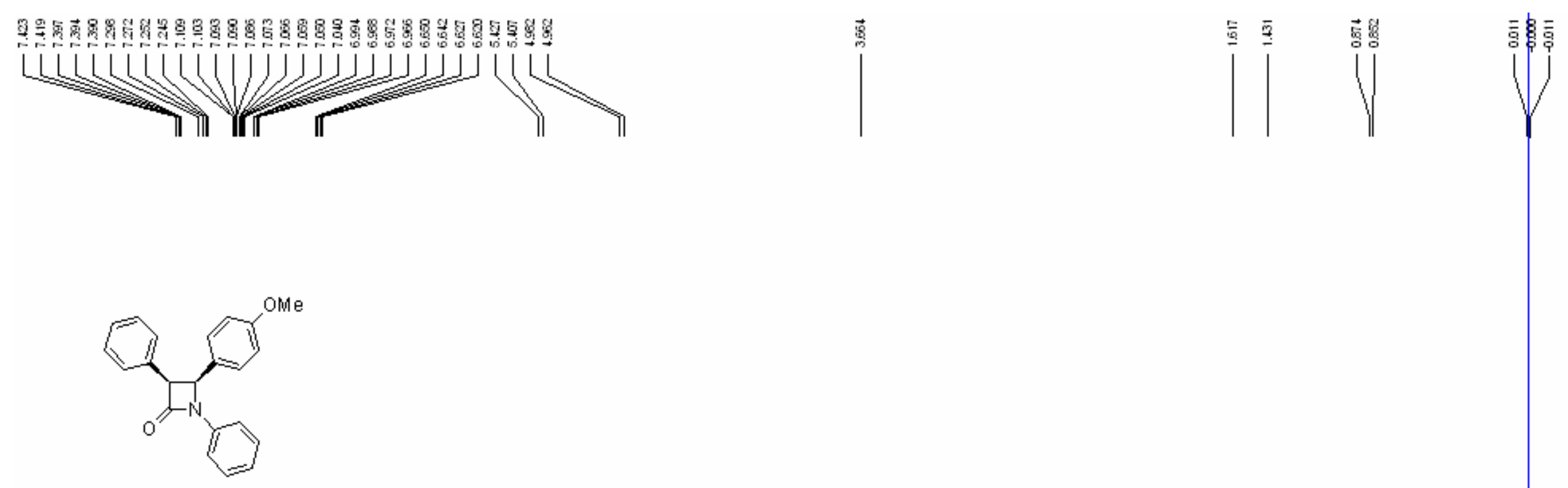

Lactam-Q1 a2

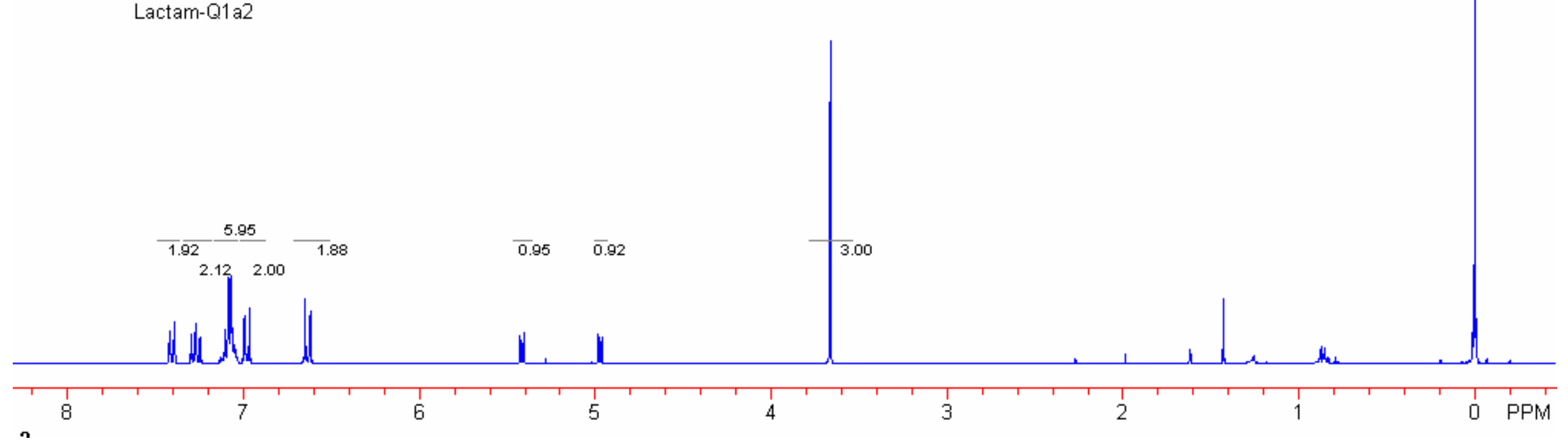




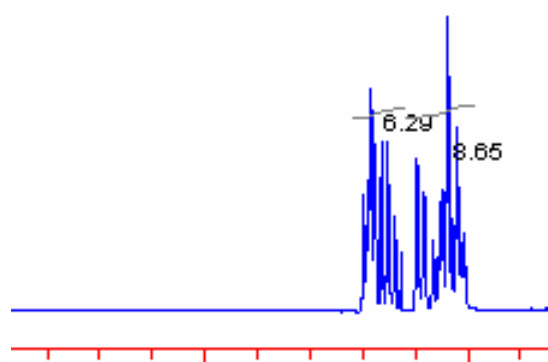



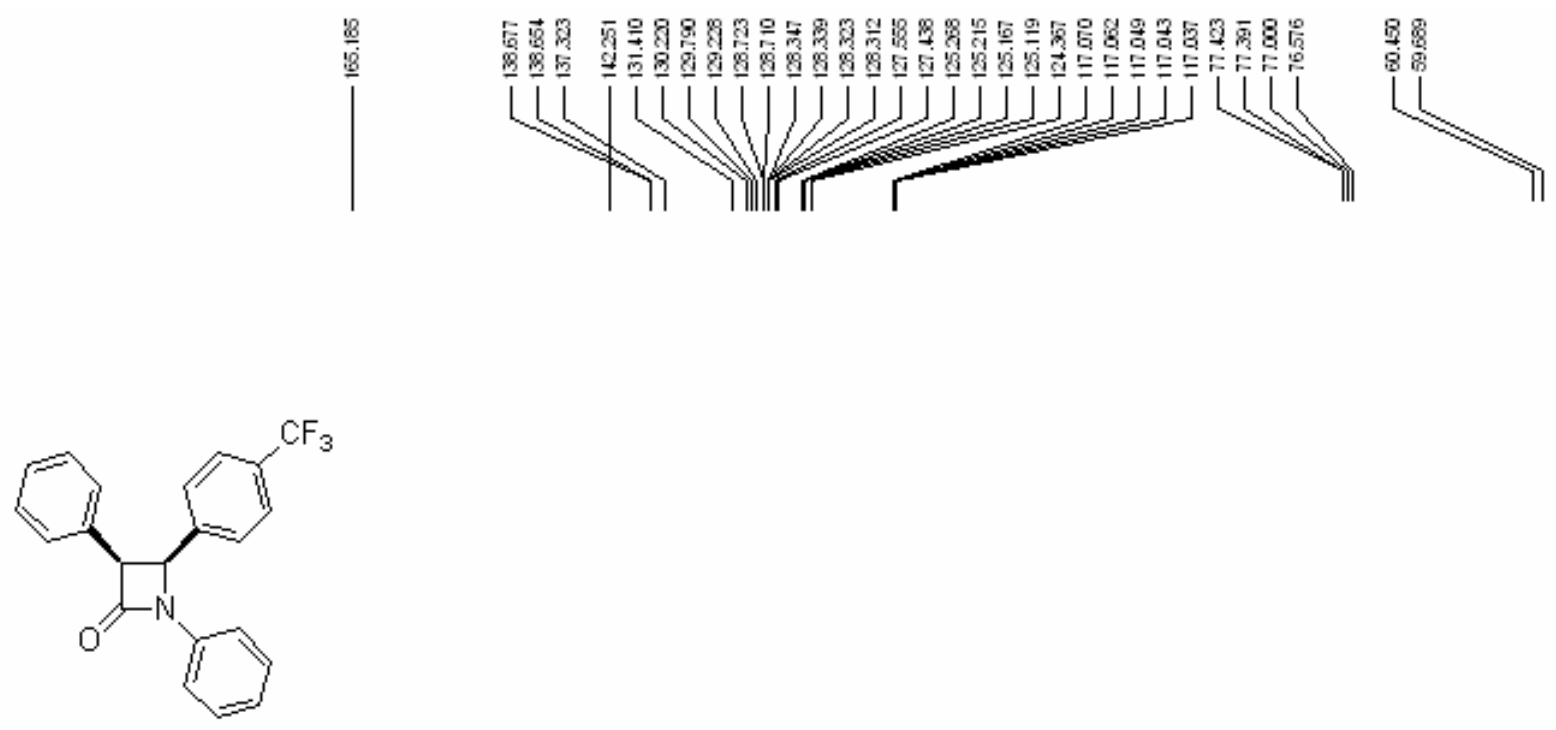

$y 4-34-52$

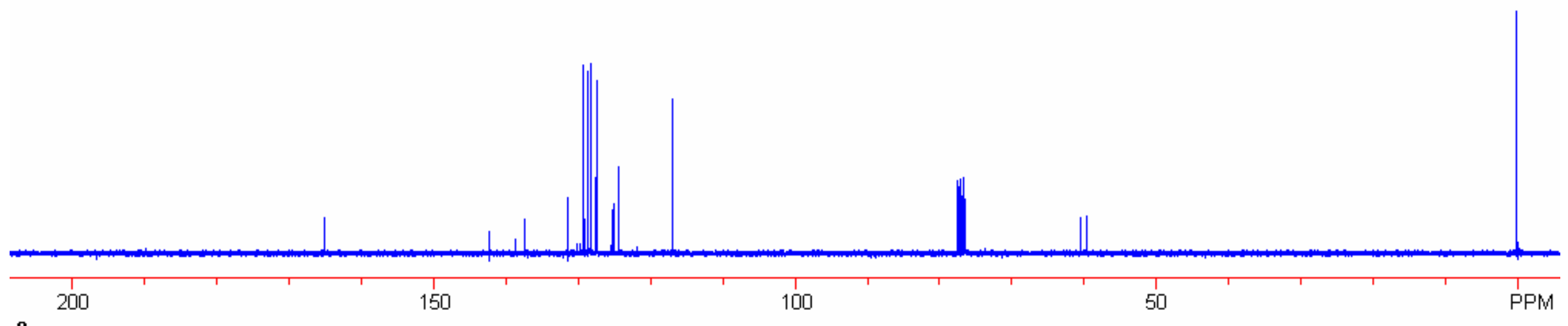




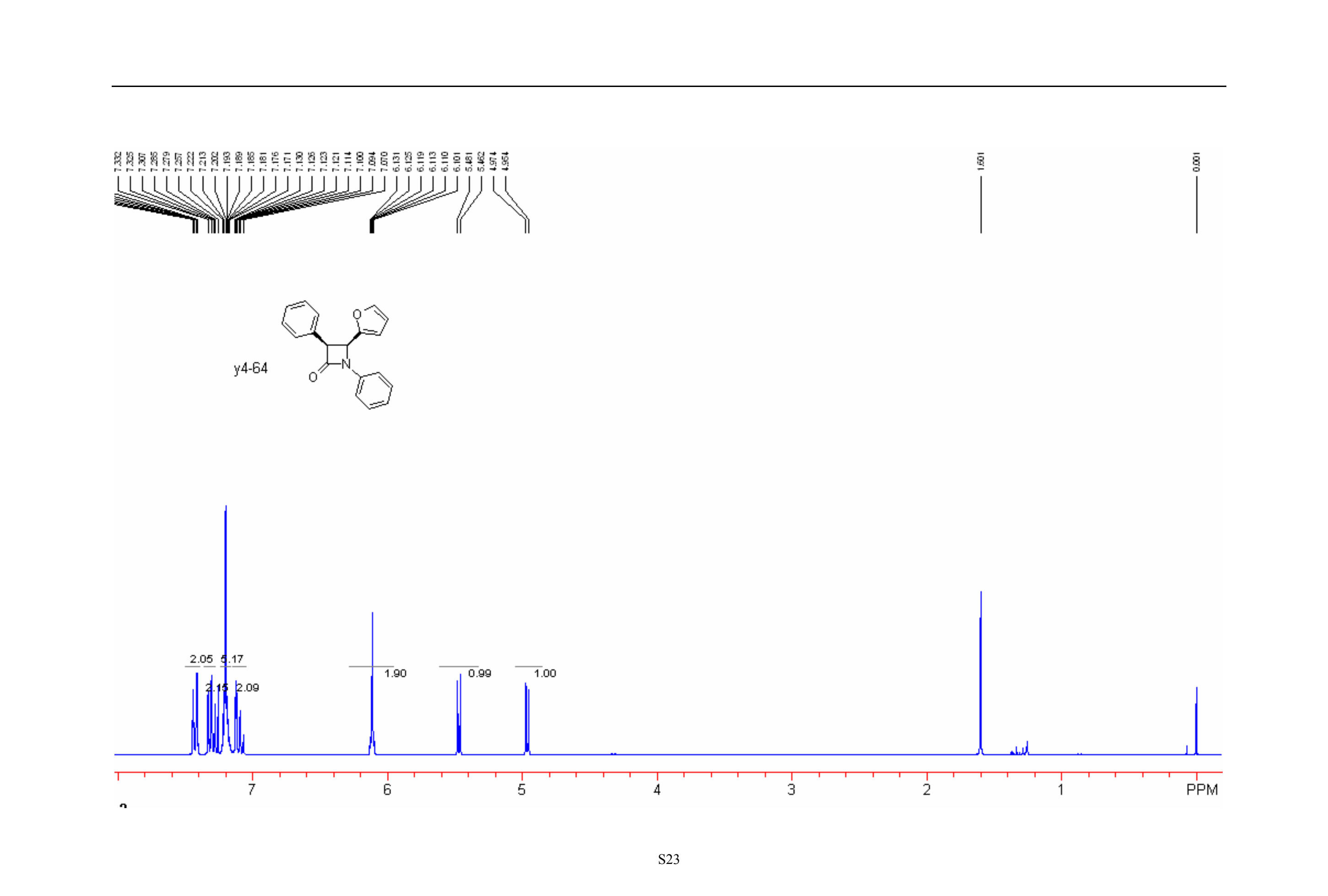



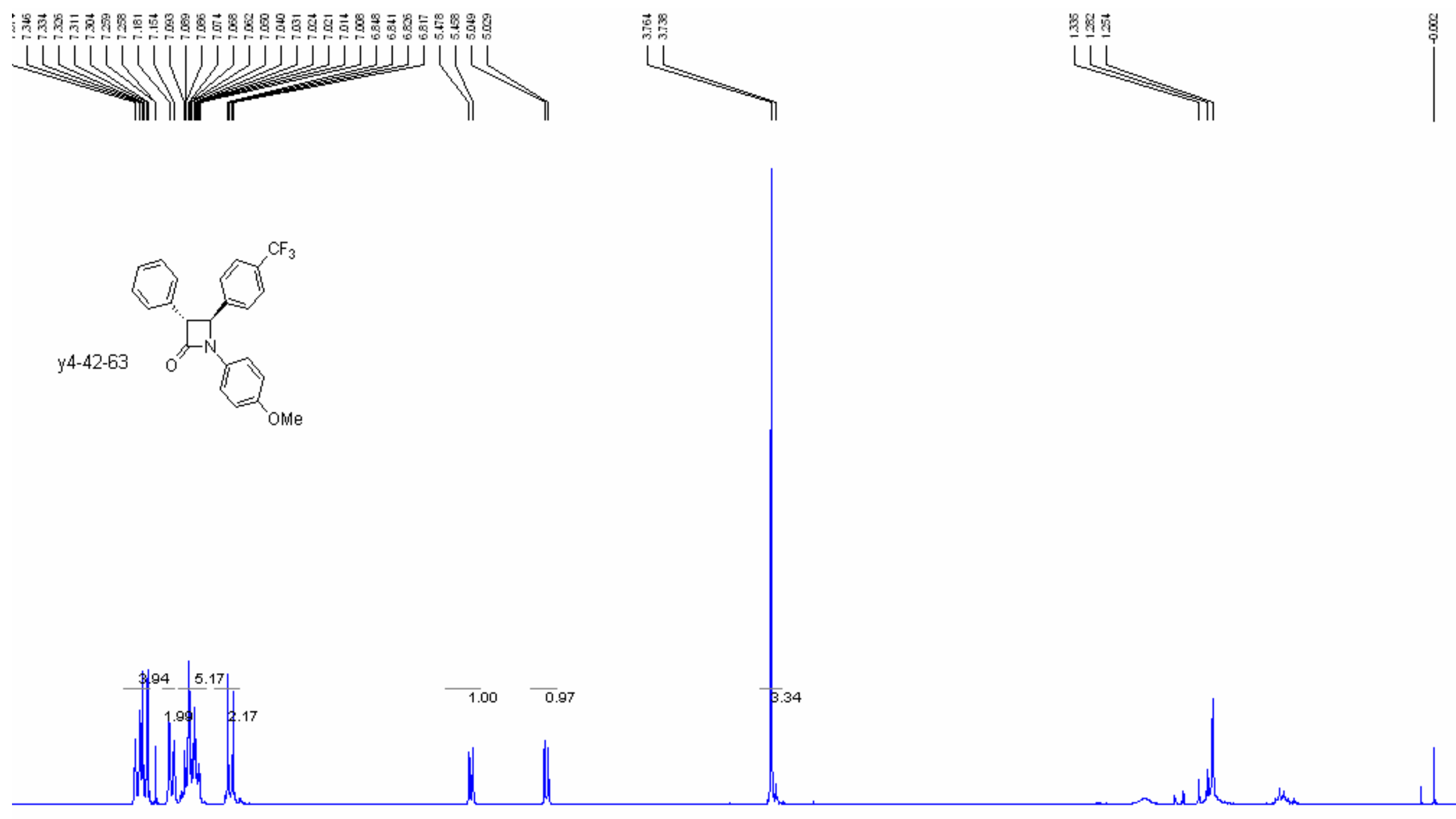

3.34
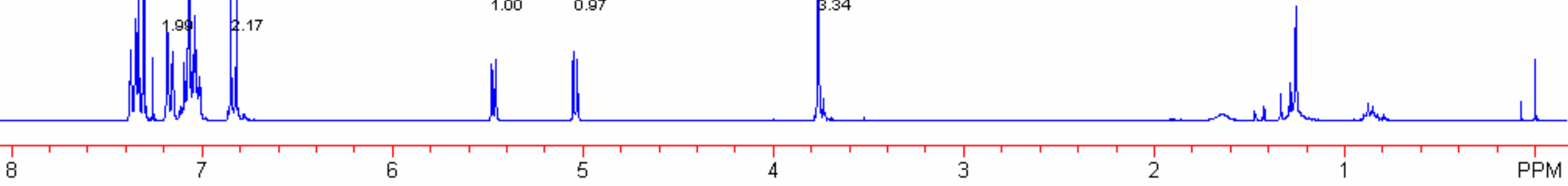

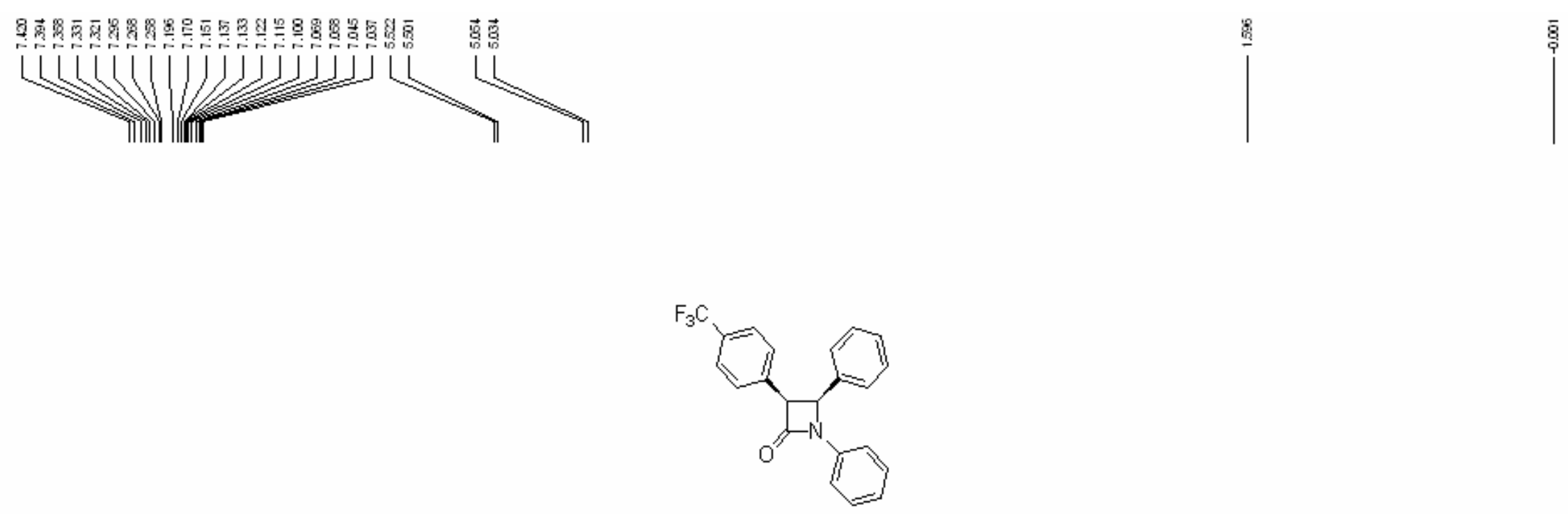

Lactam-Q4N1

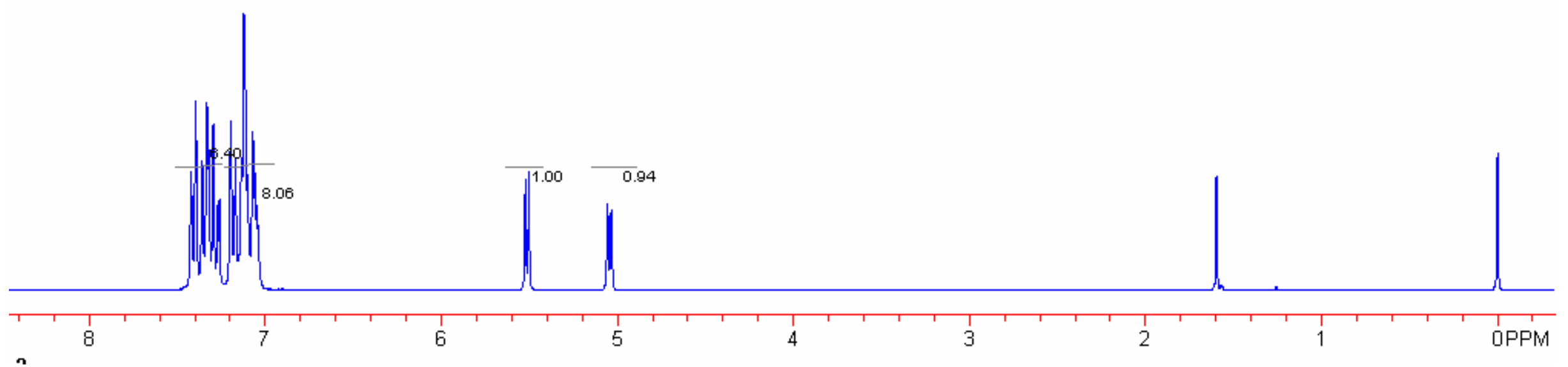



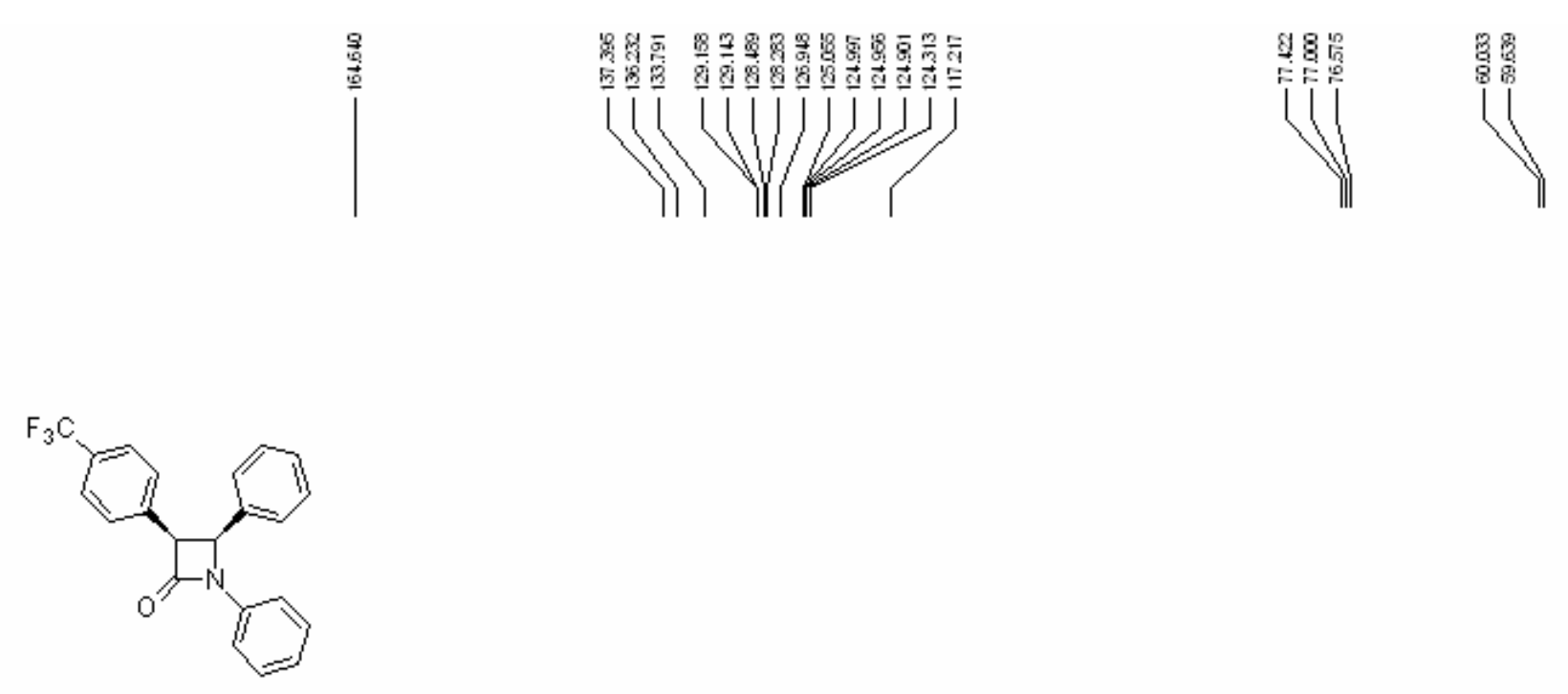

Lactam-Q4N1

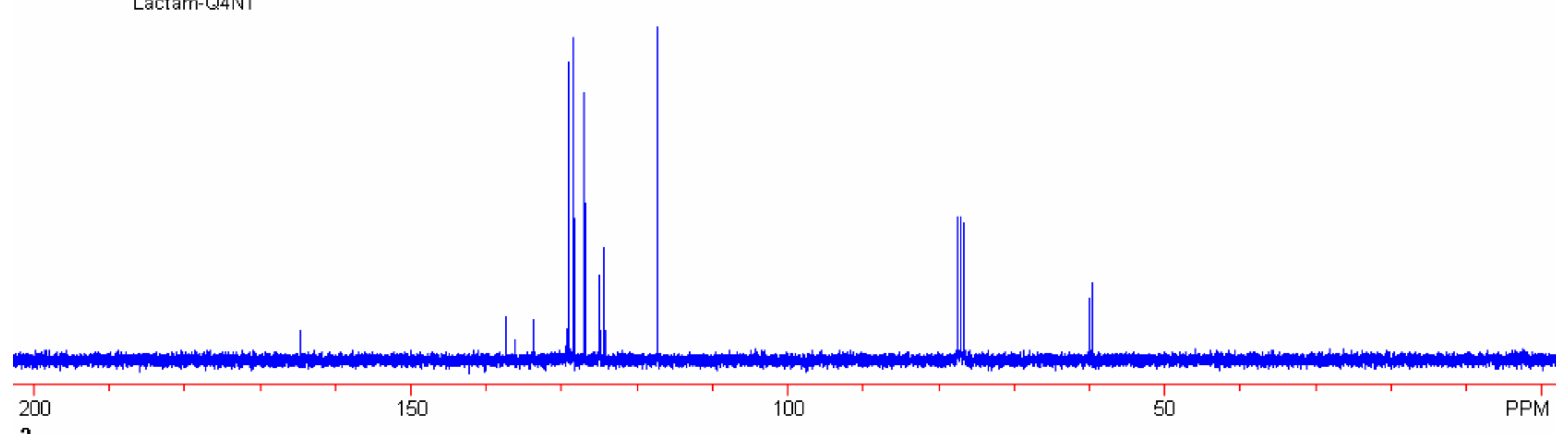




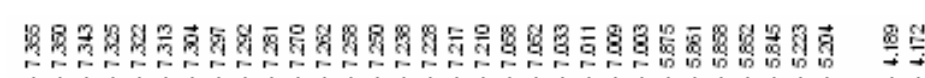
LLiLi|
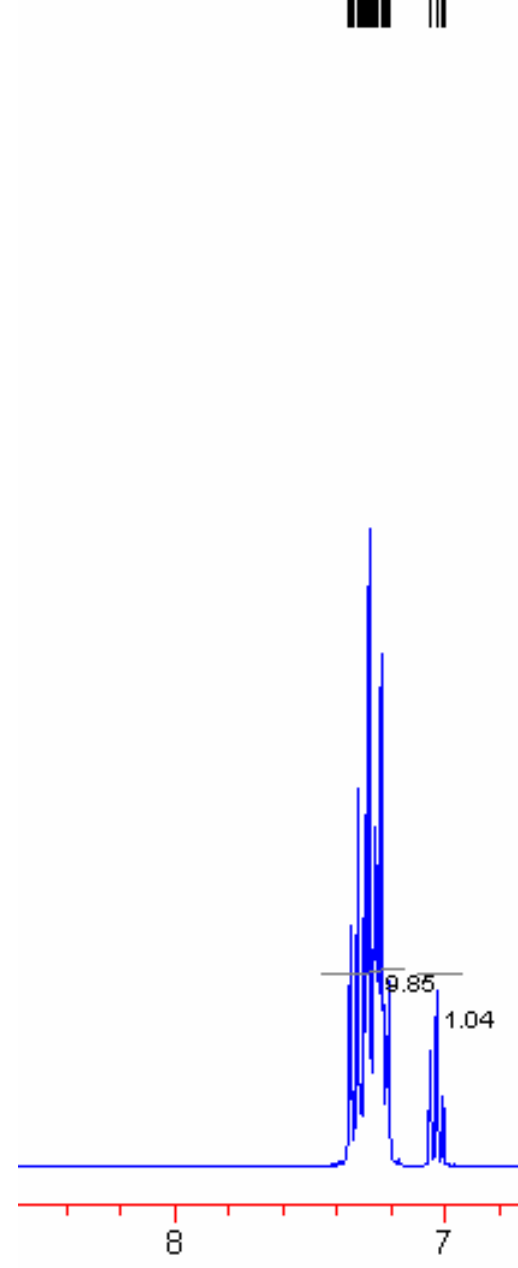

8

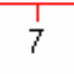

7

6
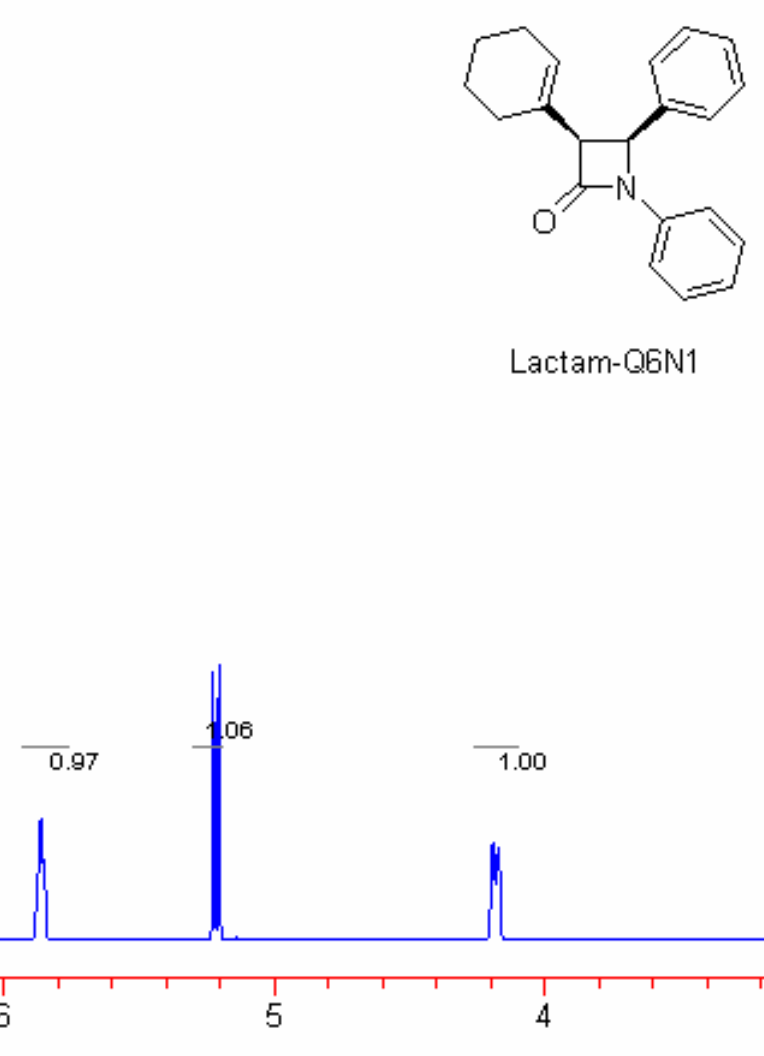

Lactam-Q6N1

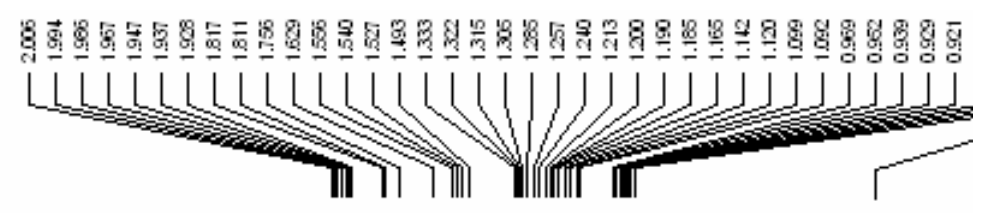

Lactam-QBN1 

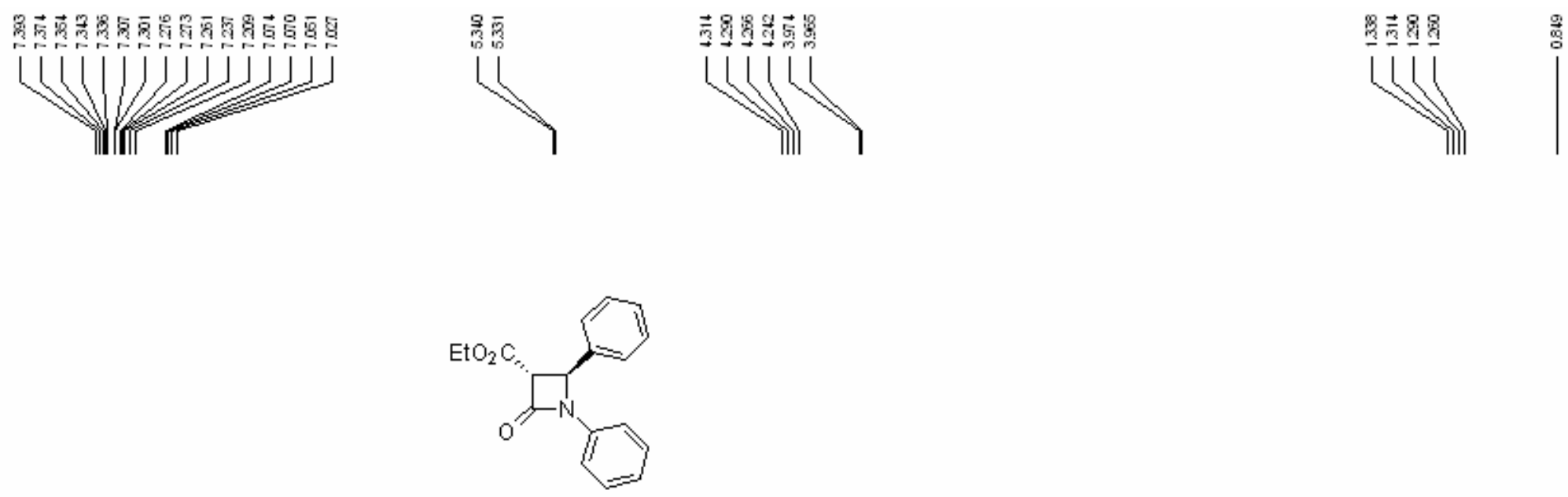

Lactam-11-81-15

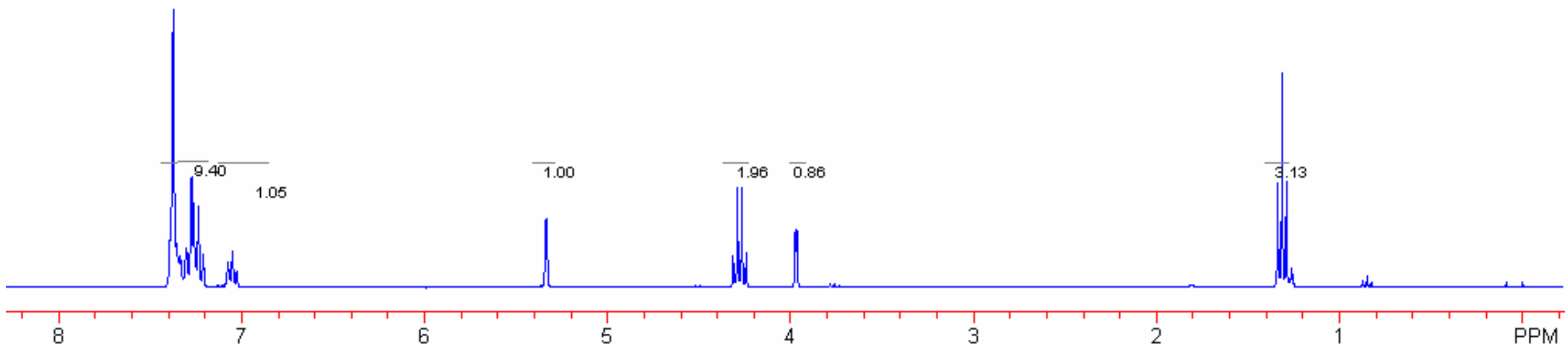

S28 

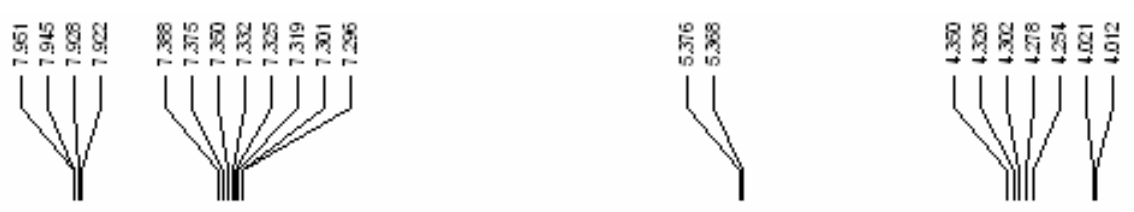

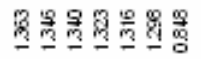

(IV)

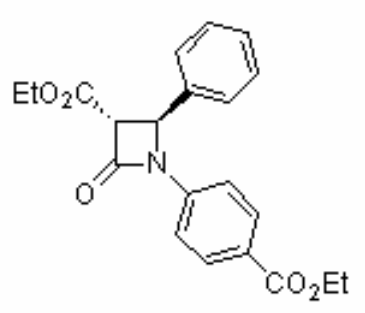

Lactam-11-85-17

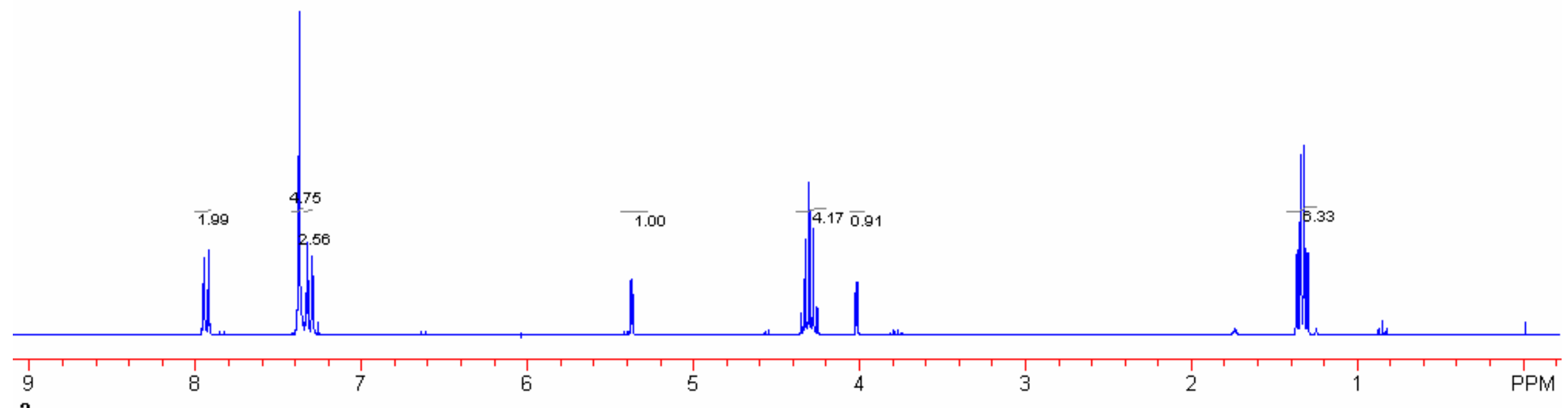



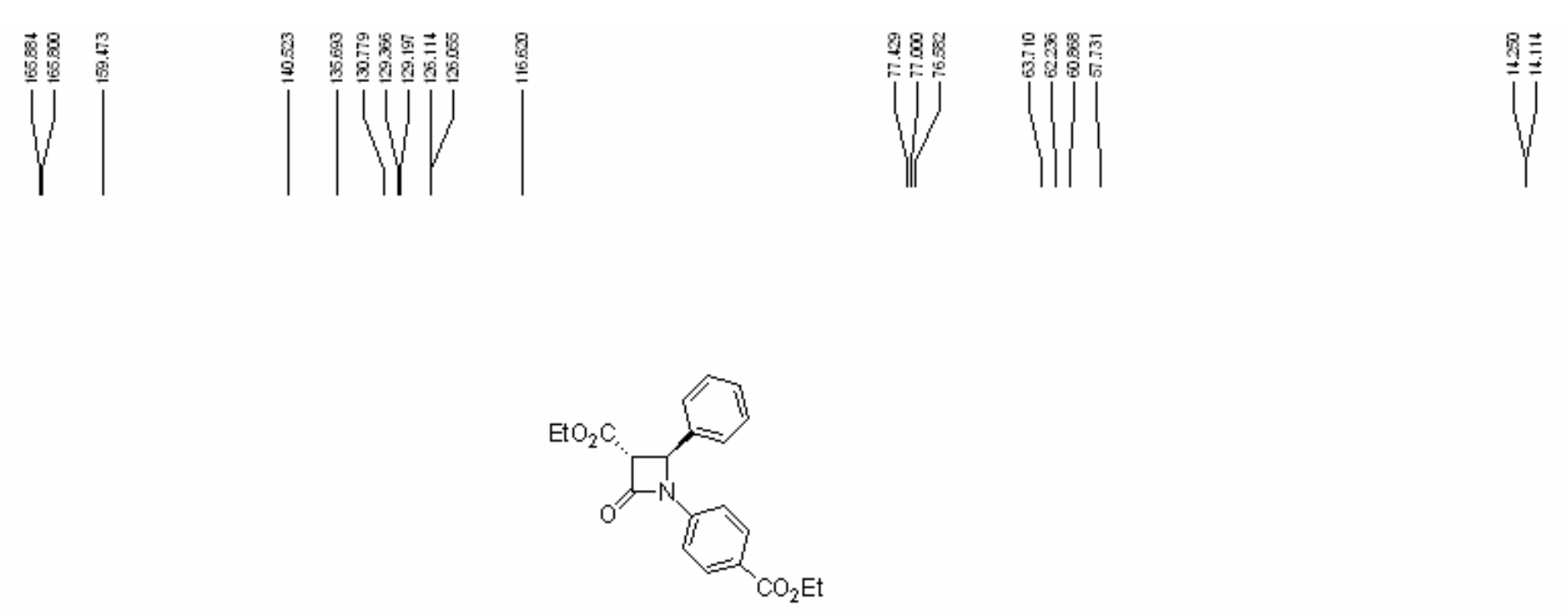

Lactam-11-85-17

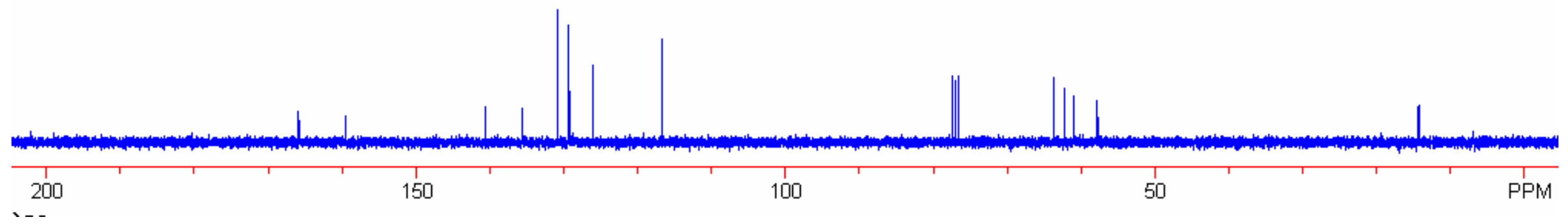




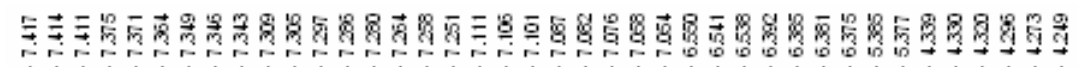

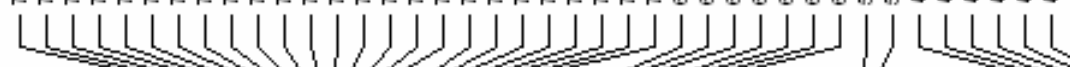
$r$
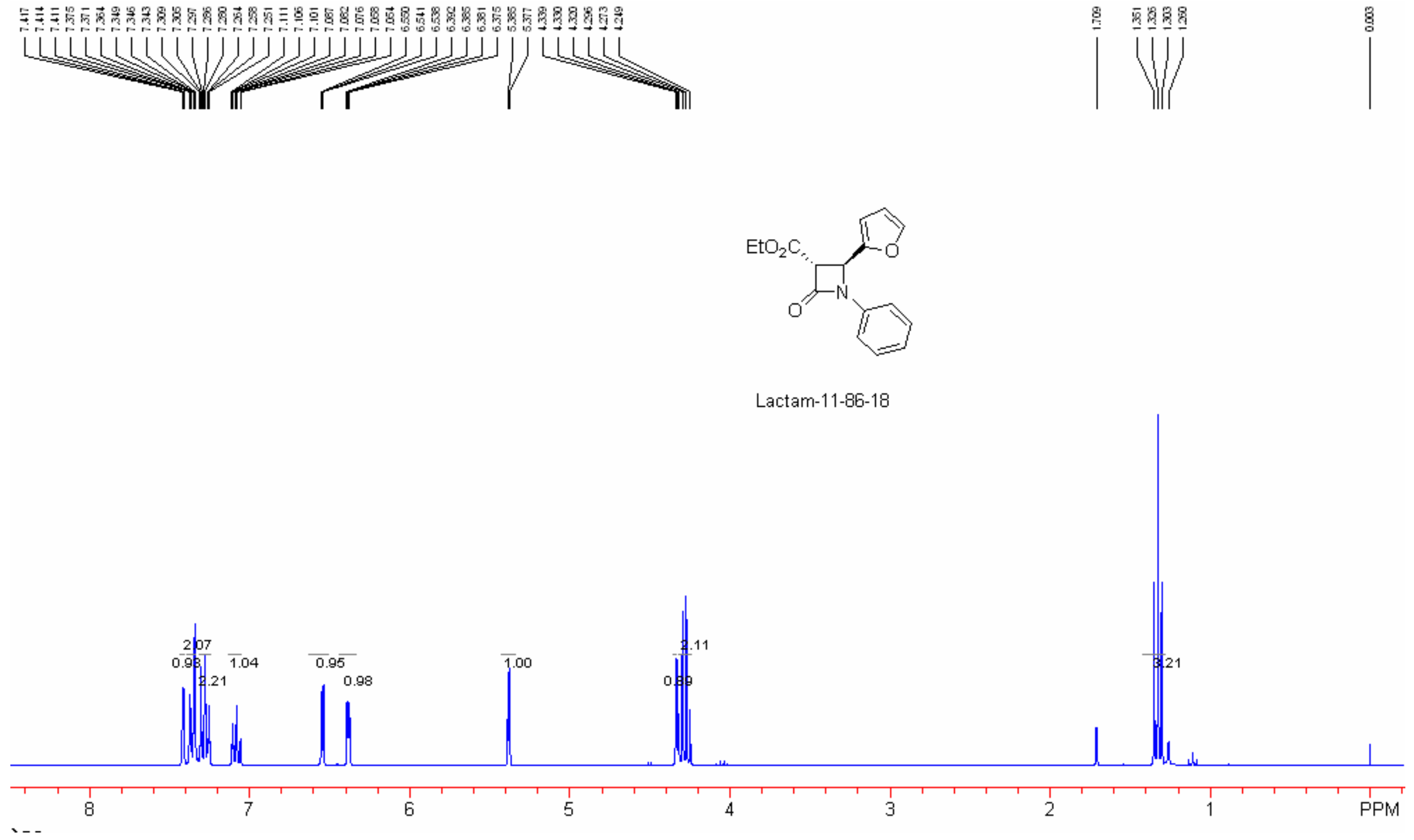


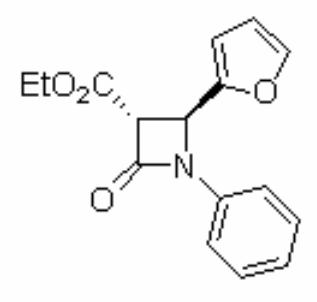

lactam-11-86-18

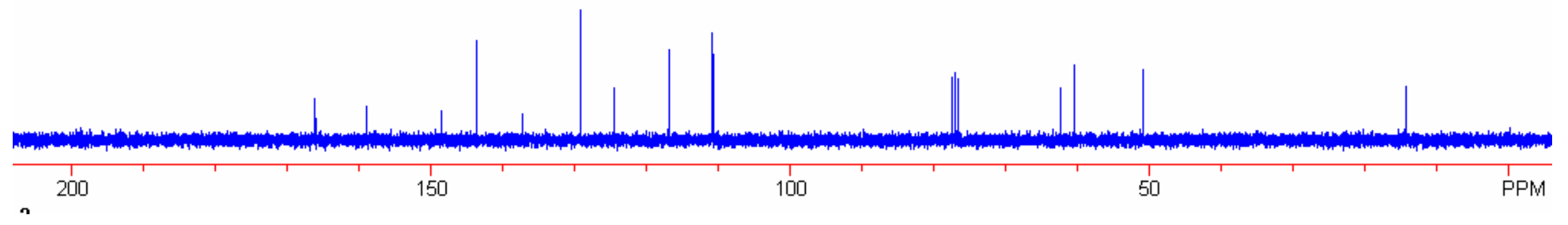




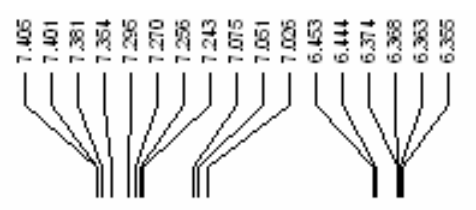

$y 12-90$

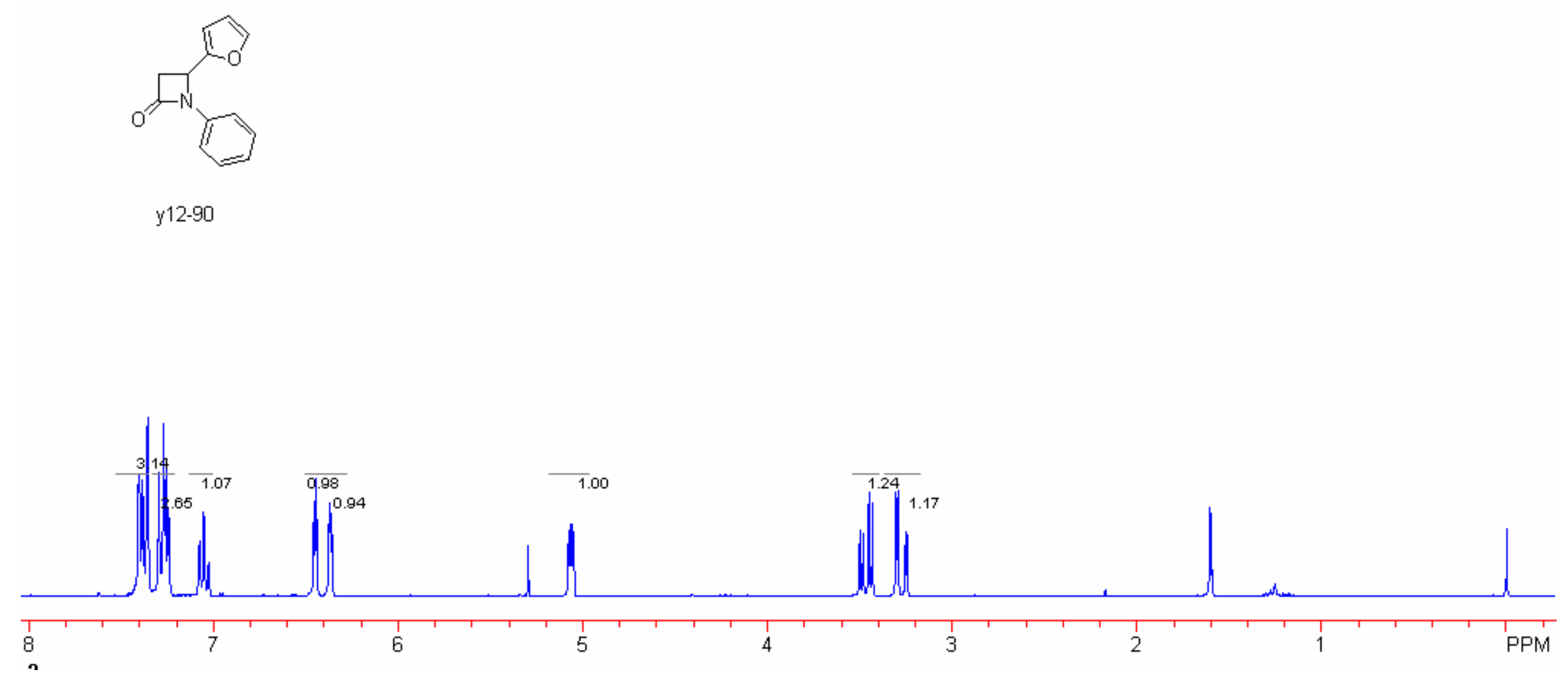

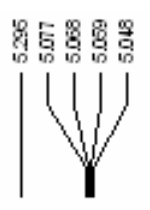

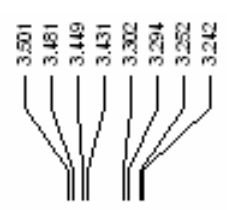

S33 


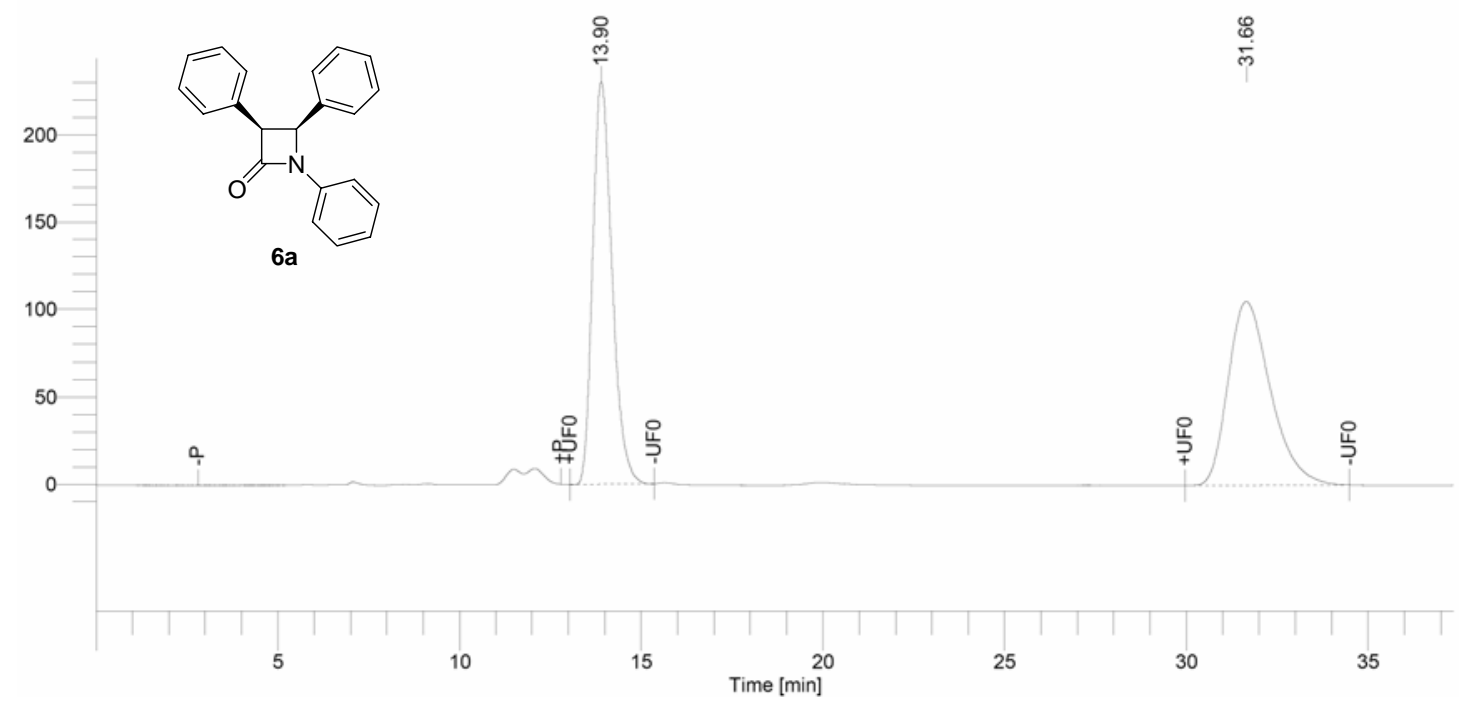

\begin{tabular}{|c|c|c|c|c|c|c|}
\hline $\begin{array}{l}\text { Time } \\
{[\mathrm{min}]}\end{array}$ & $\begin{array}{c}\text { Area } \\
{\left[\mathrm{uV}^{*} \text { sec }\right]}\end{array}$ & $\begin{array}{l}\text { Height } \\
{[\mathrm{uV}]}\end{array}$ & $\begin{array}{l}\text { Area } \\
{[\%]}\end{array}$ & $\begin{array}{c}\text { Norm. Area } \\
{[\%]}\end{array}$ & $\mathrm{BL}$ & $\begin{array}{c}\text { Adjusted } \\
\text { Amount }\end{array}$ \\
\hline \multirow{2}{*}{$\begin{array}{l}13.898 \\
31.655\end{array}$} & $\begin{array}{l}16572562.88 \\
1661090330\end{array}$ & $\begin{array}{l}447763.86 \\
204337.82\end{array}$ & $\begin{array}{l}49.94 \\
50.06\end{array}$ & 94 & MM & 16.5726 \\
\hline & 33183466.18 & 52101.68 & 100.00 & 100.00 & & 33.1835 \\
\hline
\end{tabular}

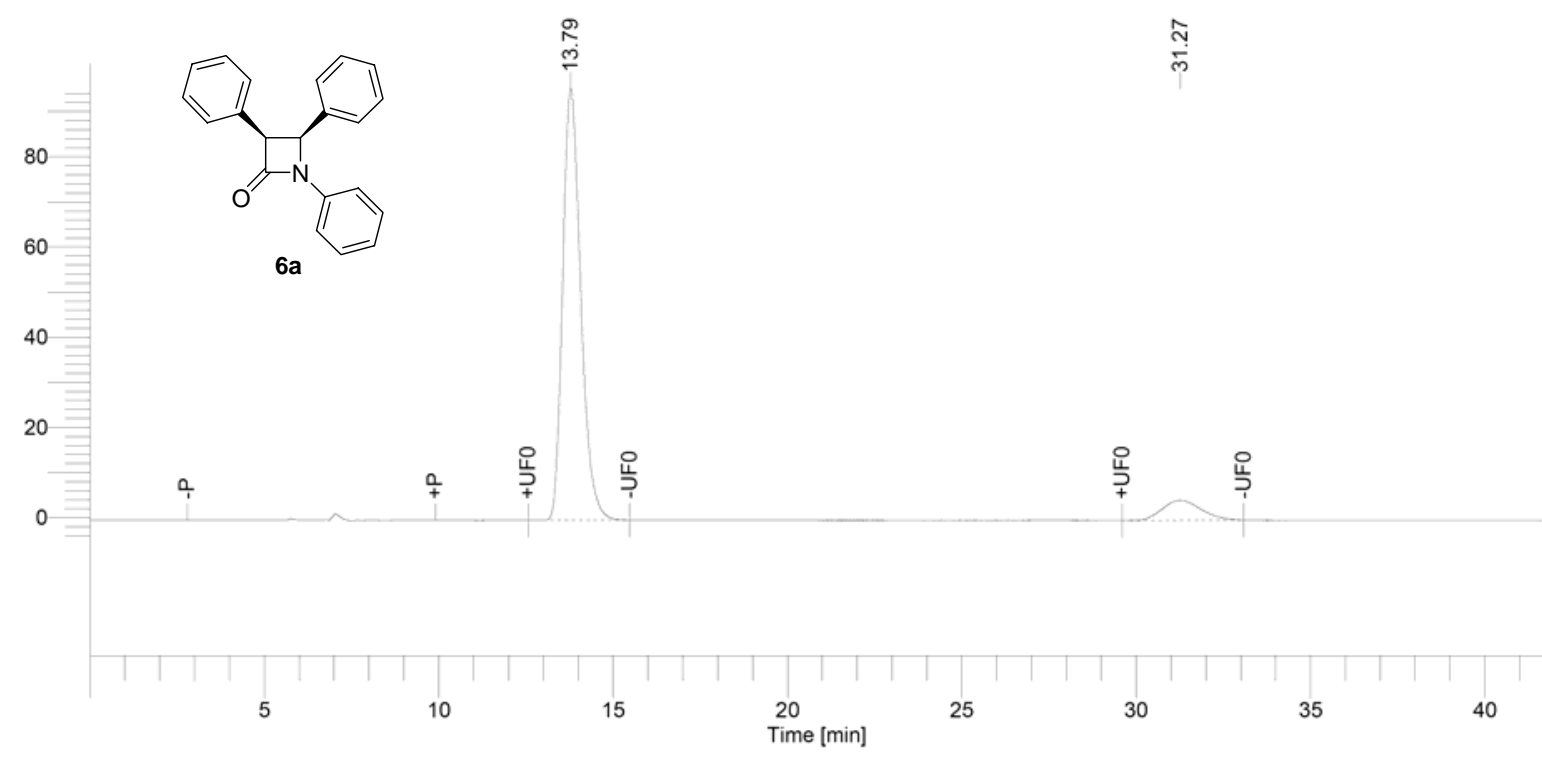

\begin{tabular}{|c|c|c|c|c|c|c|}
\hline $\begin{array}{l}\text { Time } \\
\text { [min] }\end{array}$ & $\begin{array}{c}\text { Area } \\
{\left[\mathrm{uV}^{*} \mathrm{sec}\right]}\end{array}$ & $\begin{array}{l}\text { Height } \\
\text { [uV] }\end{array}$ & $\begin{array}{l}\text { Area } \\
{[\%]}\end{array}$ & $\begin{array}{c}\text { Norm. Area } \\
{[\%]}\end{array}$ & $B L$ & $\begin{array}{l}\text { Adjusted } \\
\text { Amount }\end{array}$ \\
\hline \multirow{3}{*}{$\begin{array}{l}13.788 \\
31.268\end{array}$} & 6700296.46 & 185866.28 & 90.93 & 90.93 & $\mathrm{MM}$ & 0.10 \\
\hline & 668436.00 & 8649.58 & 9.07 & 9.07 & MM & 0.668 \\
\hline & 46 & .86 & 1000 & 100.00 & & \\
\hline
\end{tabular}




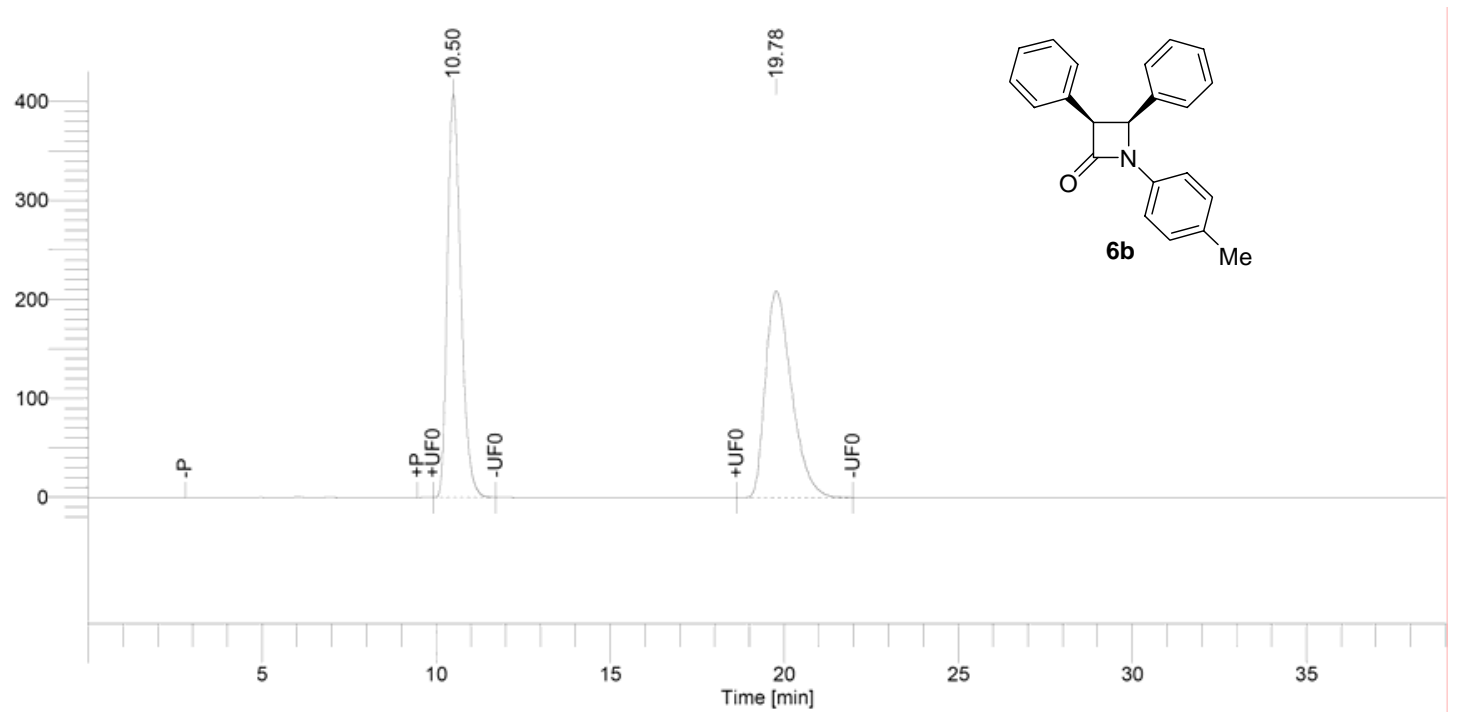

\begin{tabular}{|c|c|c|c|c|c|c|}
\hline $\begin{array}{l}\text { Time } \\
\text { [min] }\end{array}$ & $\begin{array}{c}\text { Area } \\
{\left[\mathrm{uV}^{*} \text { sec }\right]}\end{array}$ & $\begin{array}{l}\text { Height } \\
\text { [uV] }\end{array}$ & $\begin{array}{c}\text { Area } \\
{[\%]}\end{array}$ & $\begin{array}{c}\text { Norm. Area } \\
{[\%]}\end{array}$ & $\mathrm{BL}$ & $\begin{array}{l}\text { Adjusted } \\
\text { Amount }\end{array}$ \\
\hline \multirow{3}{*}{$\begin{array}{l}10.495 \\
19.777\end{array}$} & 2129 & 7917 & 49.93 & 49.93 & MM & 21.29 \\
\hline & 2135 & 406938.27 & 50.07 & 50.07 & MM & 21.35 \\
\hline & 42644346.62 & $1.20 e+06$ & 100.00 & 100.00 & & \\
\hline
\end{tabular}

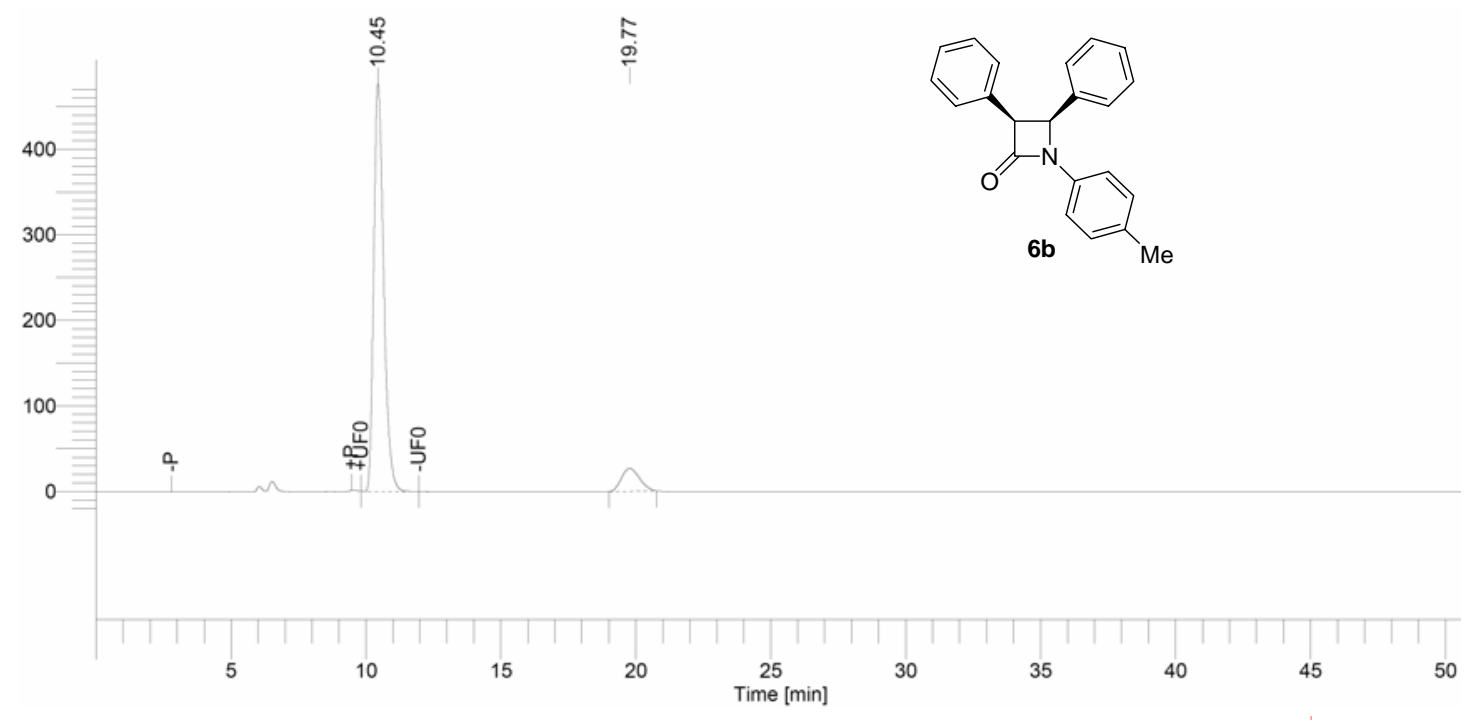

\begin{tabular}{|c|c|c|c|c|c|c|}
\hline $\begin{array}{l}\text { Time } \\
\text { [min] }\end{array}$ & $\begin{array}{c}\text { Area } \\
{\left[\mathrm{uV}^{*} \mathrm{sec}\right]}\end{array}$ & $\begin{array}{l}\text { Height } \\
\text { [uV] }\end{array}$ & $\begin{array}{l}\text { Area } \\
{[\%]}\end{array}$ & $\begin{array}{c}\text { Norm. Area } \\
{[\%]}\end{array}$ & $B L$ & $\begin{array}{l}\text { Adjusted } \\
\text { Amount }\end{array}$ \\
\hline \multirow{3}{*}{$\begin{array}{l}10.448 \\
19.774\end{array}$} & 23794730.79 & 926621.94 & 90.70 & 90.70 & MM & 23.7947 \\
\hline & 2439539.58 & 52131.31 & 9.30 & 9.30 & BB & 2.439 \\
\hline & 26234270.37 & 978753.25 & 100.00 & 100.00 & & 26.23 \\
\hline
\end{tabular}




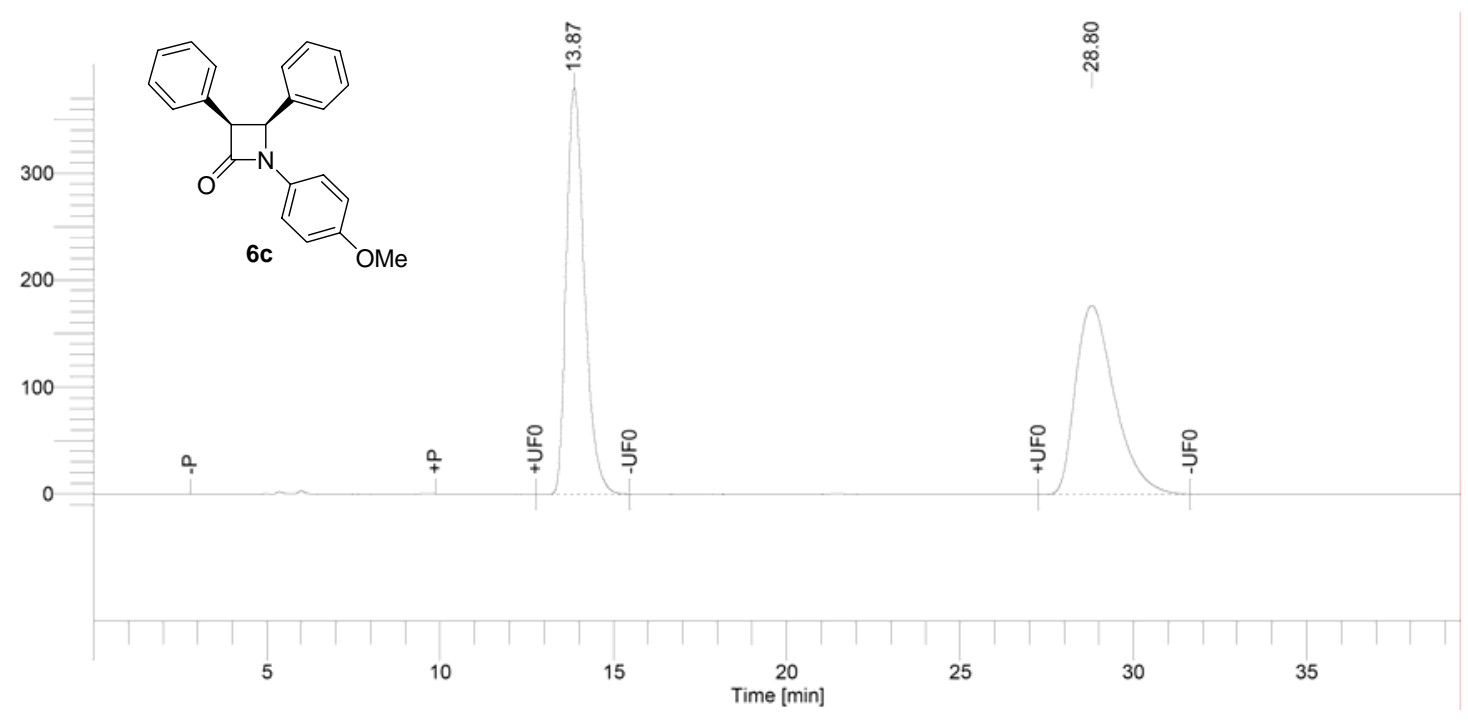

\begin{tabular}{|c|c|c|c|c|c|c|}
\hline $\begin{array}{l}\text { Time } \\
\text { [min] }\end{array}$ & $\begin{array}{c}\text { Area } \\
{\left[\mathrm{uV}^{*} \mathrm{sec}\right]}\end{array}$ & $\begin{array}{l}\text { Height } \\
{[\mathrm{uV}]}\end{array}$ & $\begin{array}{c}\text { Area } \\
{[\%]}\end{array}$ & $\begin{array}{c}\text { Norm. Area } \\
{[\%]}\end{array}$ & $B L$ & $\begin{array}{l}\text { Adjusted } \\
\text { Amount }\end{array}$ \\
\hline \multirow{3}{*}{$\begin{array}{l}13.870 \\
28.802\end{array}$} & 2695 & 739625.16 & 50.01 & 50.01 & MM & 26.9555 \\
\hline & 26939817.17 & 343566.63 & 49.99 & 49.99 & MM & 26.9398 \\
\hline & 53895295.47 & $1.08 e+06$ & 100.00 & 100.00 & & 53.8953 \\
\hline
\end{tabular}

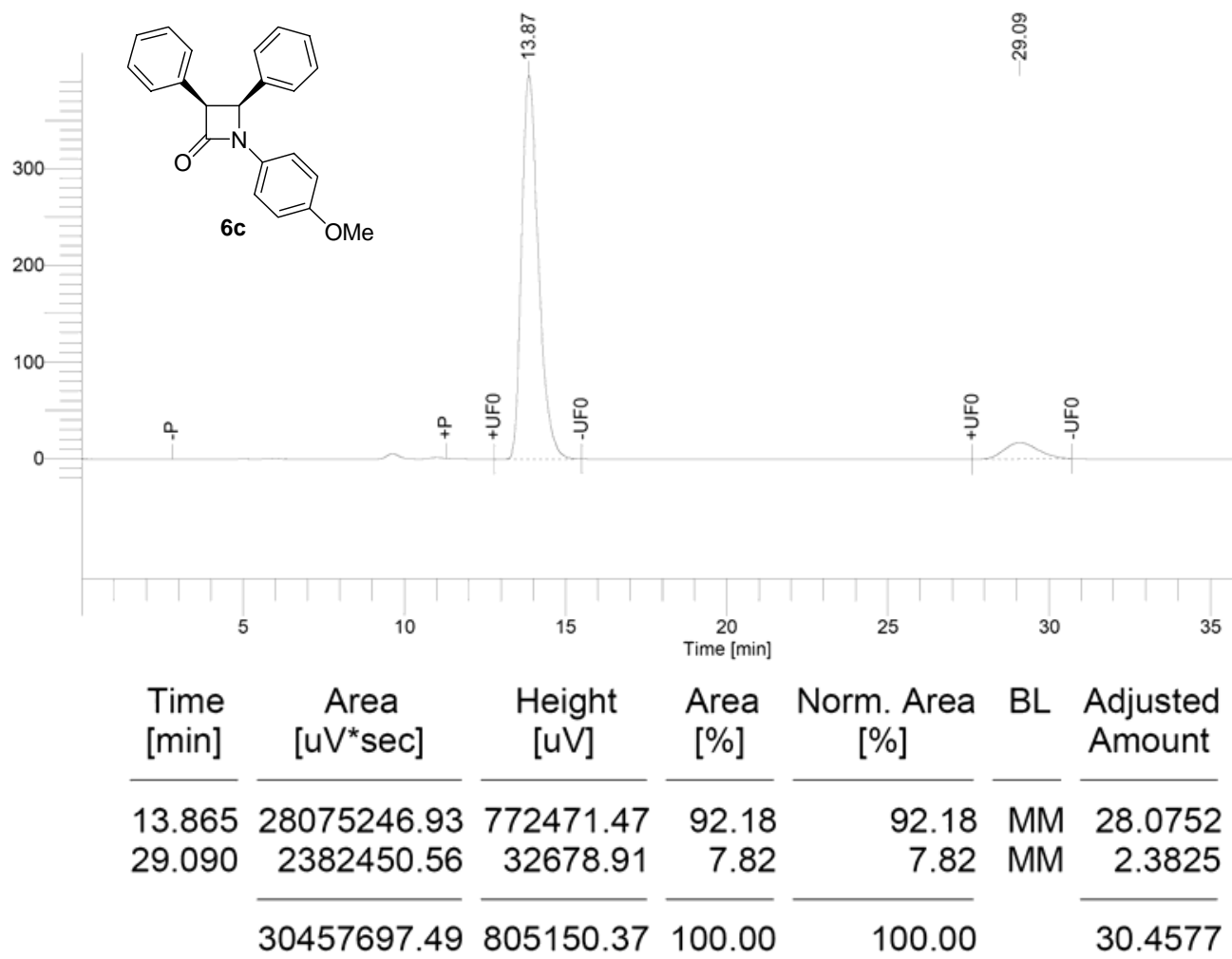




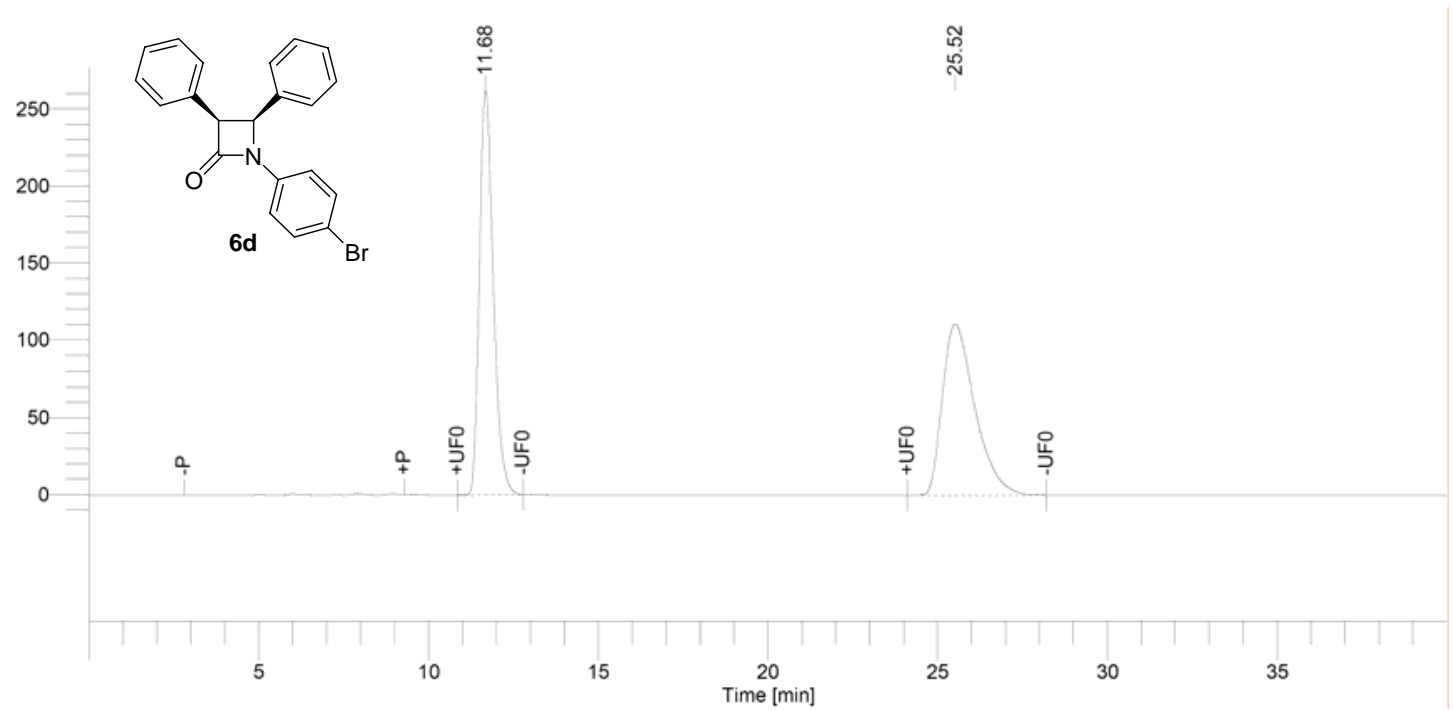

\begin{tabular}{|c|c|c|c|c|c|c|}
\hline $\begin{array}{l}\text { Time } \\
\text { [min] }\end{array}$ & $\begin{array}{c}\text { Area } \\
{\left[\mathrm{uV}^{*} \text { sec }\right]}\end{array}$ & $\begin{array}{l}\text { Height } \\
\text { [uV] }\end{array}$ & $\begin{array}{l}\text { Area } \\
{[\%]}\end{array}$ & $\begin{array}{c}\text { Norm. Area } \\
{[\%]}\end{array}$ & $B L$ & $\begin{array}{l}\text { Adjusted } \\
\text { Amount }\end{array}$ \\
\hline 11.680 & 14505564.04 & 509096.47 & 50.20 & 50.20 & MM & 14.50 \\
\hline \multirow[t]{2}{*}{25.517} & 14392759.27 & 215631.62 & 49.80 & 49.80 & MM & 14.39 \\
\hline & & .10 & 000 & 100.00 & & \\
\hline
\end{tabular}

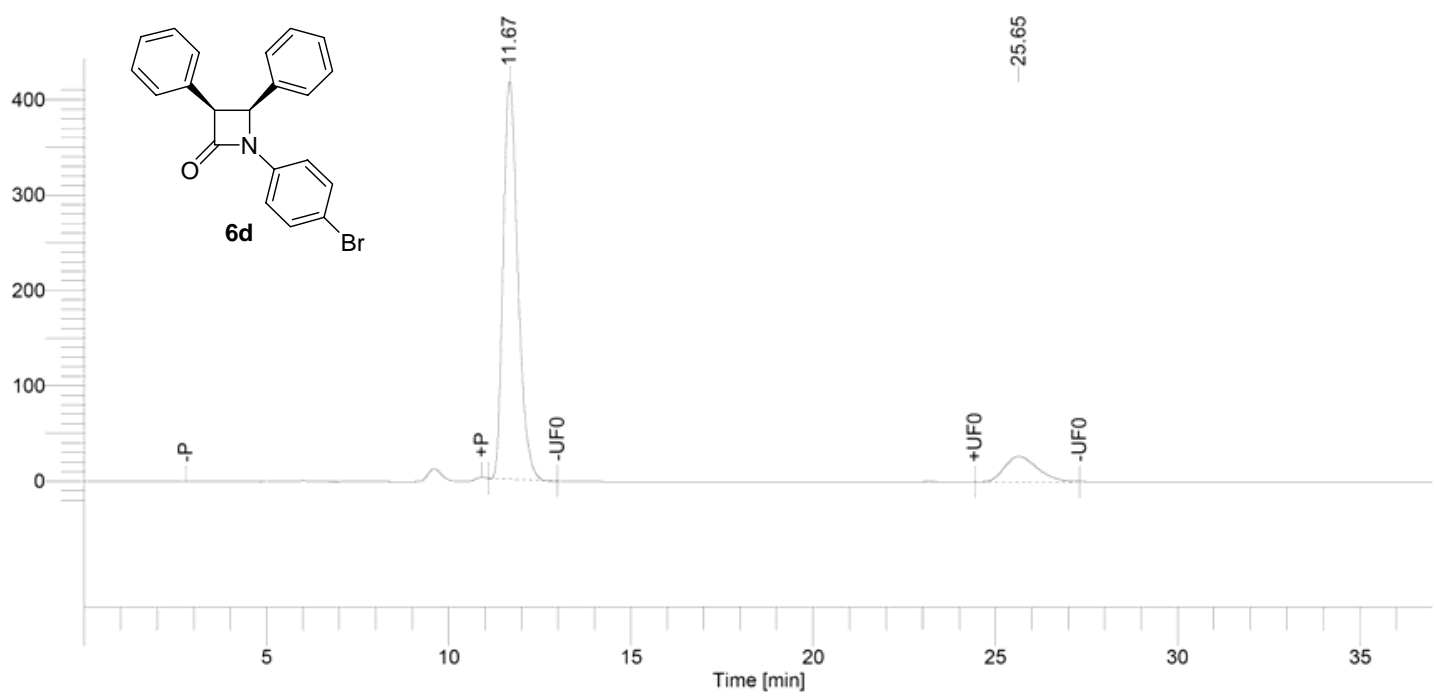

\begin{tabular}{|c|c|c|c|c|c|c|}
\hline $\begin{array}{l}\text { Time } \\
\text { [min] }\end{array}$ & $\begin{array}{c}\text { Area } \\
{\left[\mathrm{uV}^{*} \mathrm{sec}\right]}\end{array}$ & $\begin{array}{l}\text { Height } \\
{[\mathrm{uV}]}\end{array}$ & $\begin{array}{l}\text { Area } \\
{[\%]}\end{array}$ & $\begin{array}{c}\text { Norm. Area } \\
{[\%]}\end{array}$ & $\mathrm{BL}$ & $\begin{array}{l}\text { Adjusted } \\
\text { Amount }\end{array}$ \\
\hline 11.672 & 22702409.54 & 810447.59 & 87.54 & 87.54 & MM & 22.7024 \\
\hline 25.645 & 3232797.20 & 51480.25 & 12.46 & 12.46 & MM & 3.2328 \\
\hline
\end{tabular}




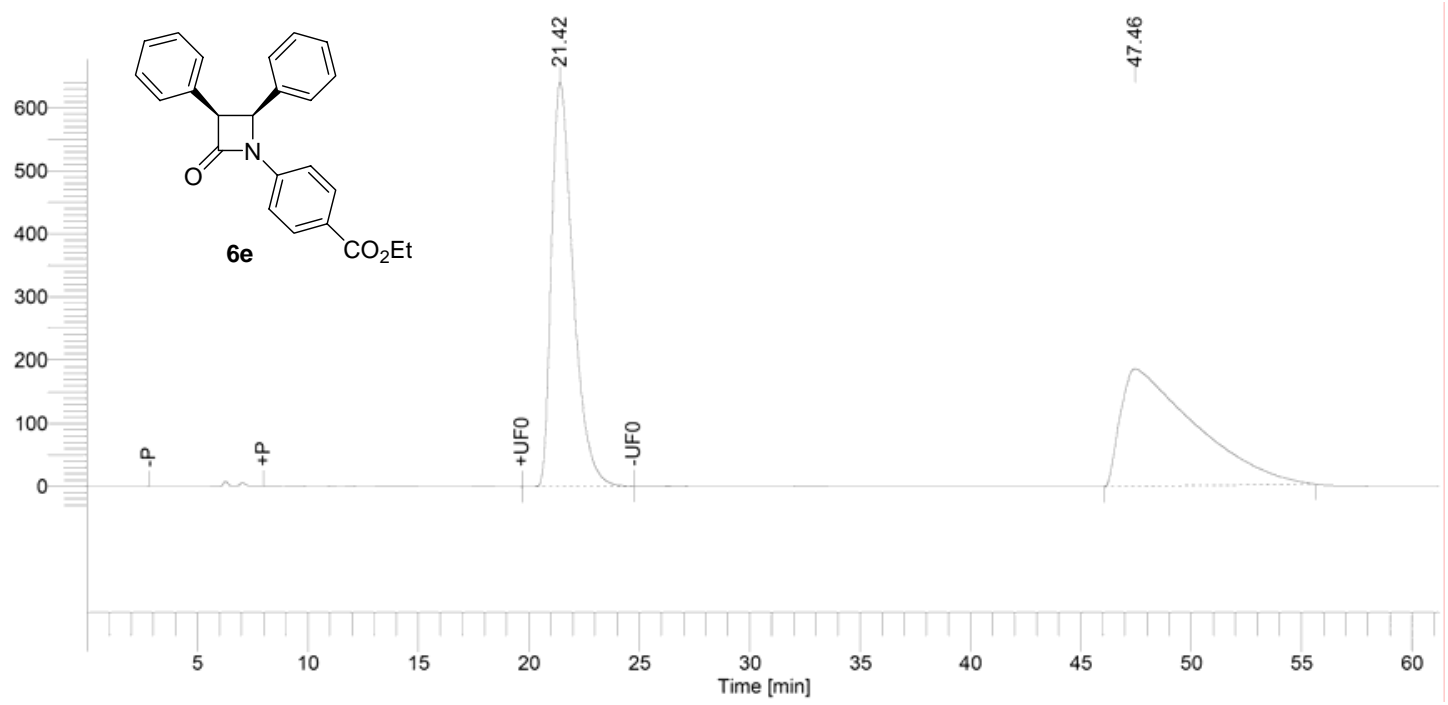

\begin{tabular}{|c|c|c|c|c|c|c|}
\hline $\begin{array}{l}\text { Time } \\
\text { [min] }\end{array}$ & $\begin{array}{c}\text { Area } \\
{\left[\mathrm{uV}^{*} \text { sec }\right]}\end{array}$ & $\begin{array}{l}\text { Height } \\
{[\mathrm{uV}]}\end{array}$ & $\begin{array}{c}\text { Area } \\
{[\%]}\end{array}$ & $\begin{array}{c}\text { Norm. Area } \\
{[\%]}\end{array}$ & $\mathrm{BL}$ & $\begin{array}{l}\text { Adjusted } \\
\text { Amount }\end{array}$ \\
\hline \multirow{3}{*}{$\begin{array}{l}21.417 \\
47.461\end{array}$} & 85164003.94 & $1.25 e+06$ & 50.11 & 50.11 & MM & 85.16 \\
\hline & 84803075.02 & 362622.56 & 49.89 & 49.89 & BB & 84.803 \\
\hline & +08 & $1.61 e+06$ & 100.00 & 100.00 & & 96 \\
\hline
\end{tabular}

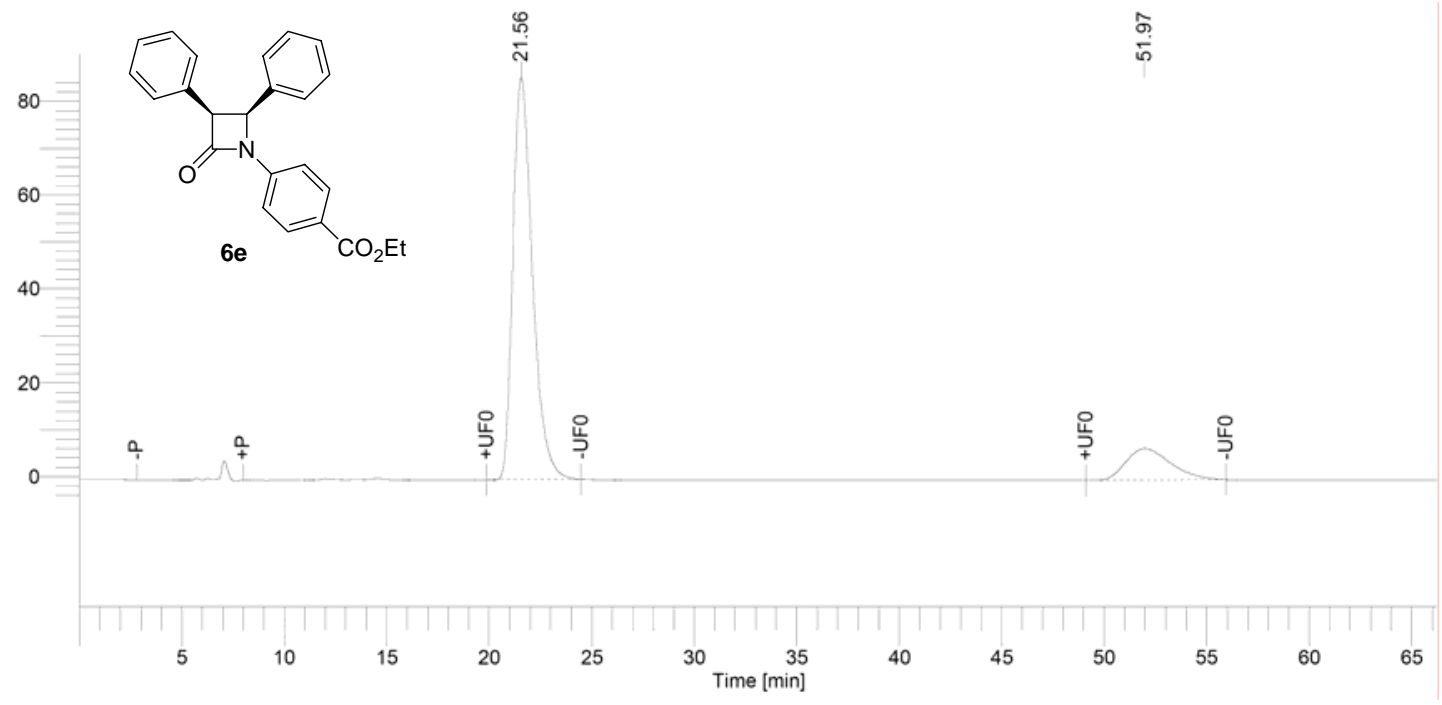

\begin{tabular}{|c|c|c|c|c|c|c|}
\hline $\begin{array}{l}\text { Time } \\
\text { [min] }\end{array}$ & $\begin{array}{c}\text { Area } \\
{\left[\mathrm{uV}^{*} \mathrm{sec}\right]}\end{array}$ & $\begin{array}{l}\text { Height } \\
{[\mathrm{uV}]}\end{array}$ & $\begin{array}{l}\text { Area } \\
{[\%]}\end{array}$ & $\begin{array}{c}\text { Norm. Area } \\
{[\%]}\end{array}$ & $\mathrm{BL}$ & $\begin{array}{l}\text { Adjusted } \\
\text { Amount }\end{array}$ \\
\hline 21.558 & 11040415.73 & 166749.33 & 84.77 & 84.77 & MM & 11.04 \\
\hline \multirow[t]{2}{*}{51.970} & 1983520.21 & 13092.47 & 15.23 & 15.23 & MM & 1.9835 \\
\hline & 13023935.94 & 179841.80 & 100.00 & 100.00 & & 13 \\
\hline
\end{tabular}




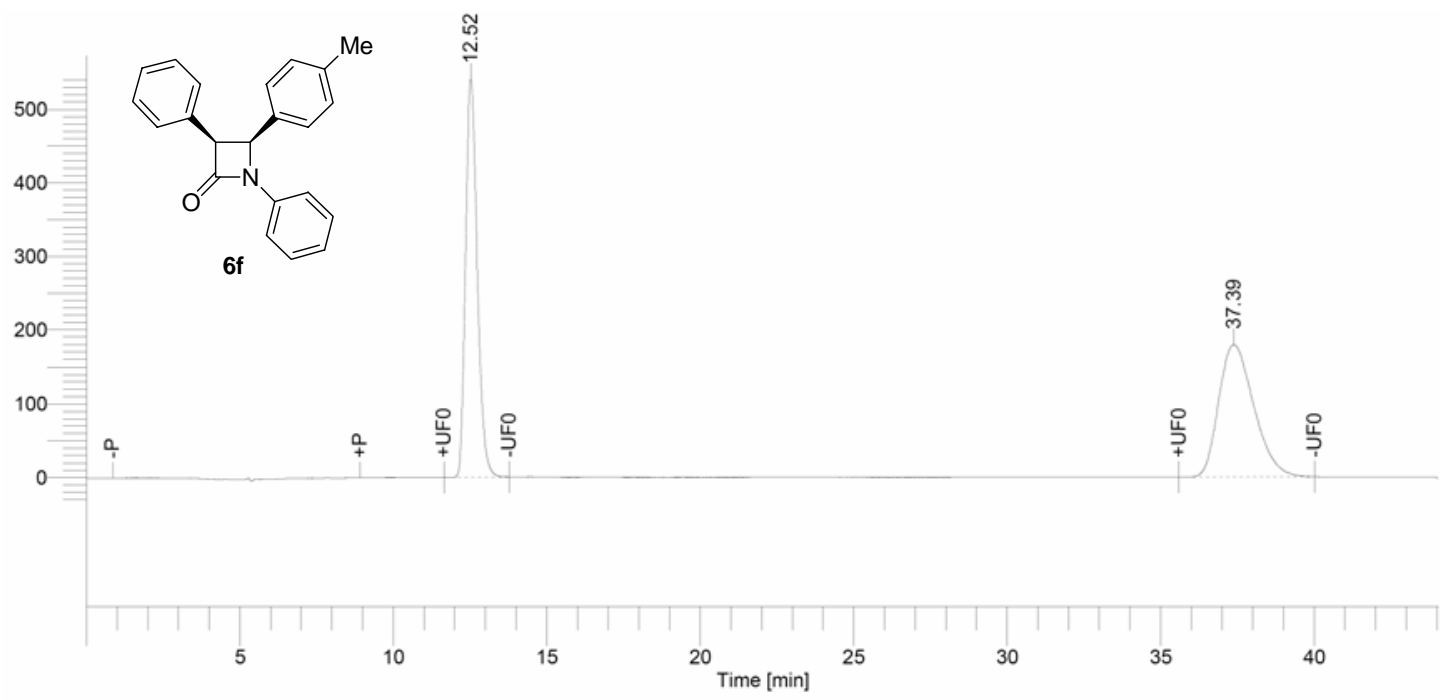

\begin{tabular}{|c|c|c|c|c|c|c|}
\hline $\begin{array}{l}\text { Time } \\
\text { [min] }\end{array}$ & $\begin{array}{c}\text { Area } \\
{\left[\mathrm{uV}^{*} \mathrm{sec}\right]}\end{array}$ & $\begin{array}{l}\text { Height } \\
{[\mathrm{uV}]}\end{array}$ & $\begin{array}{l}\text { Area } \\
{[\%]}\end{array}$ & $\begin{array}{c}\text { Norm. Area } \\
{[\%]}\end{array}$ & $\mathrm{BL}$ & $\begin{array}{c}\text { Adjusted } \\
\text { Amount }\end{array}$ \\
\hline \multirow{3}{*}{$\begin{array}{l}12.522 \\
37.388\end{array}$} & 142 & 540 & 49 & 49 & MM & 903 \\
\hline & 14300670.99 & 179135.88 & 50.02 & 50.02 & MM & 14.3007 \\
\hline & 45 & 79 & .00 & 100.00 & & \\
\hline
\end{tabular}

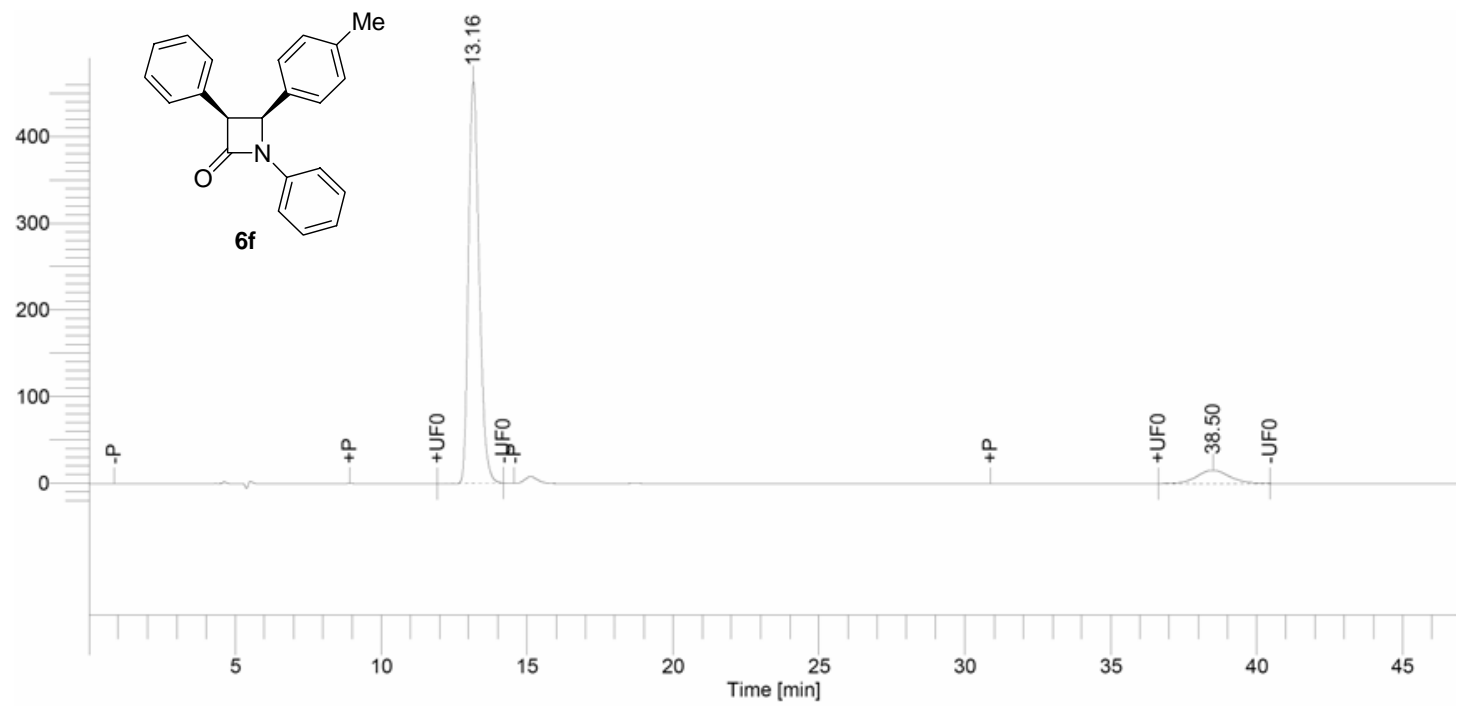

\begin{tabular}{|c|c|c|c|c|c|c|}
\hline $\begin{array}{l}\text { Time } \\
\text { [min] }\end{array}$ & $\begin{array}{c}\text { Area } \\
{\left[\mathrm{uV}^{*} \mathrm{sec}\right]}\end{array}$ & $\begin{array}{l}\text { Height } \\
{[\mathrm{uV}]}\end{array}$ & $\begin{array}{l}\text { Area } \\
{[\%]}\end{array}$ & $\begin{array}{c}\text { Norm. Area } \\
{[\%]}\end{array}$ & $\mathrm{BL}$ & $\begin{array}{l}\text { Adjusted } \\
\text { Amount }\end{array}$ \\
\hline & 118 & 463 & 91.00 & 91.00 & MM & 01 \\
\hline \multirow{2}{*}{38.500} & 1167581.82 & 15179.49 & 9.00 & 9.00 & MM & 1.1676 \\
\hline & 977 & 7826 & 00.00 & 00.00 & & 777 \\
\hline
\end{tabular}



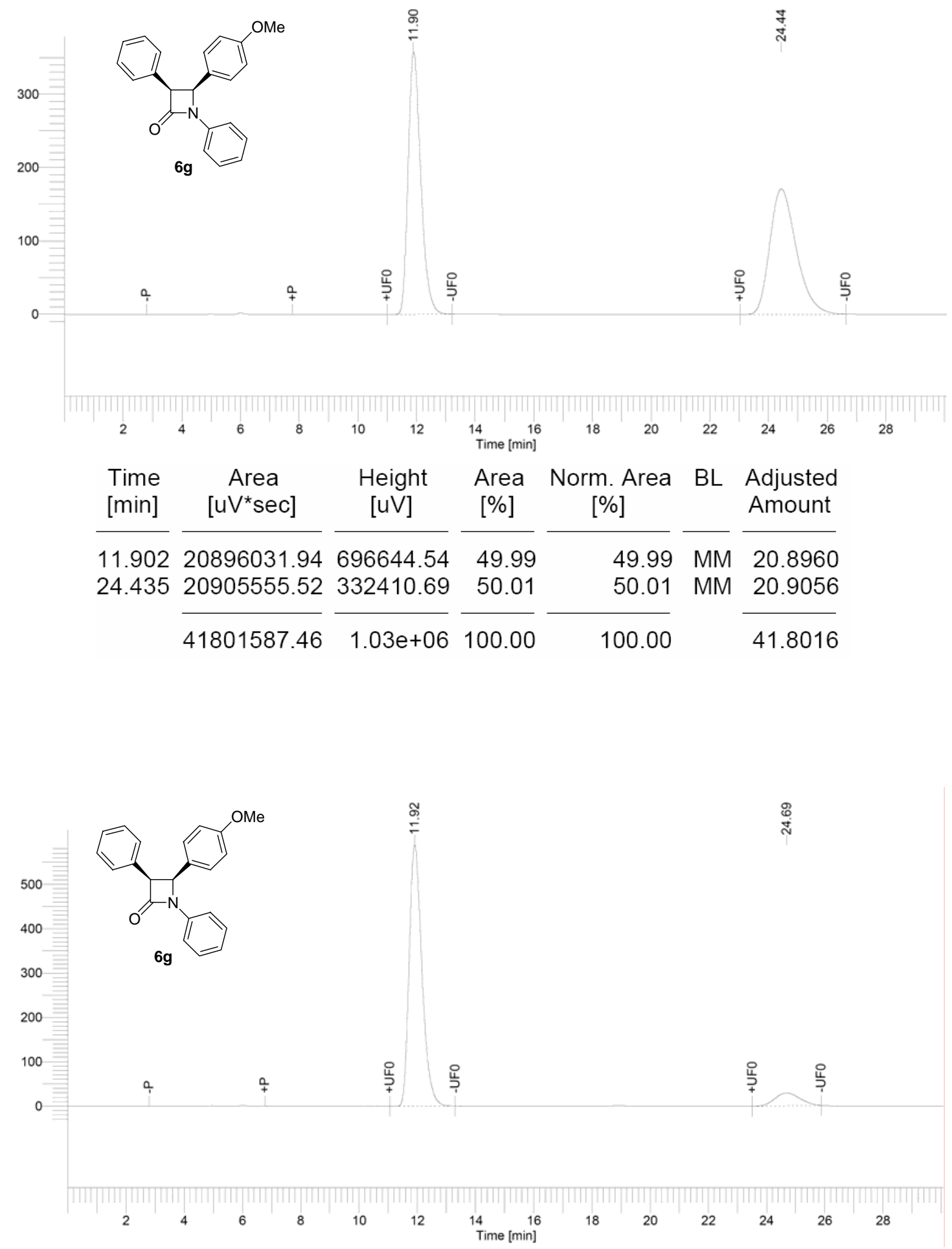

\begin{tabular}{|c|c|c|c|c|c|c|}
\hline $\begin{array}{l}\text { Time } \\
\text { [min] }\end{array}$ & $\begin{array}{c}\text { Area } \\
{\left[\mathrm{uV}^{*} \text { sec }\right]}\end{array}$ & $\begin{array}{l}\text { Height } \\
{[\mathrm{uV}]}\end{array}$ & $\begin{array}{c}\text { Area } \\
{[\%]}\end{array}$ & $\begin{array}{c}\text { Norm. Area } \\
{[\%]}\end{array}$ & $B L$ & $\begin{array}{c}\text { Adjusted } \\
\text { Amount }\end{array}$ \\
\hline \multirow{3}{*}{$\begin{array}{l}11.917 \\
24.692\end{array}$} & 34985048.95 & $1.15 \mathrm{e}+06$ & 91.26 & 91.26 & MM & 34.9850 \\
\hline & 3352523.32 & 56395.25 & 8.74 & 8.74 & MM & 3.3525 \\
\hline & 38337572.26 & $1.20 \mathrm{e}+06$ & 100.00 & 100.00 & & 38.3376 \\
\hline
\end{tabular}




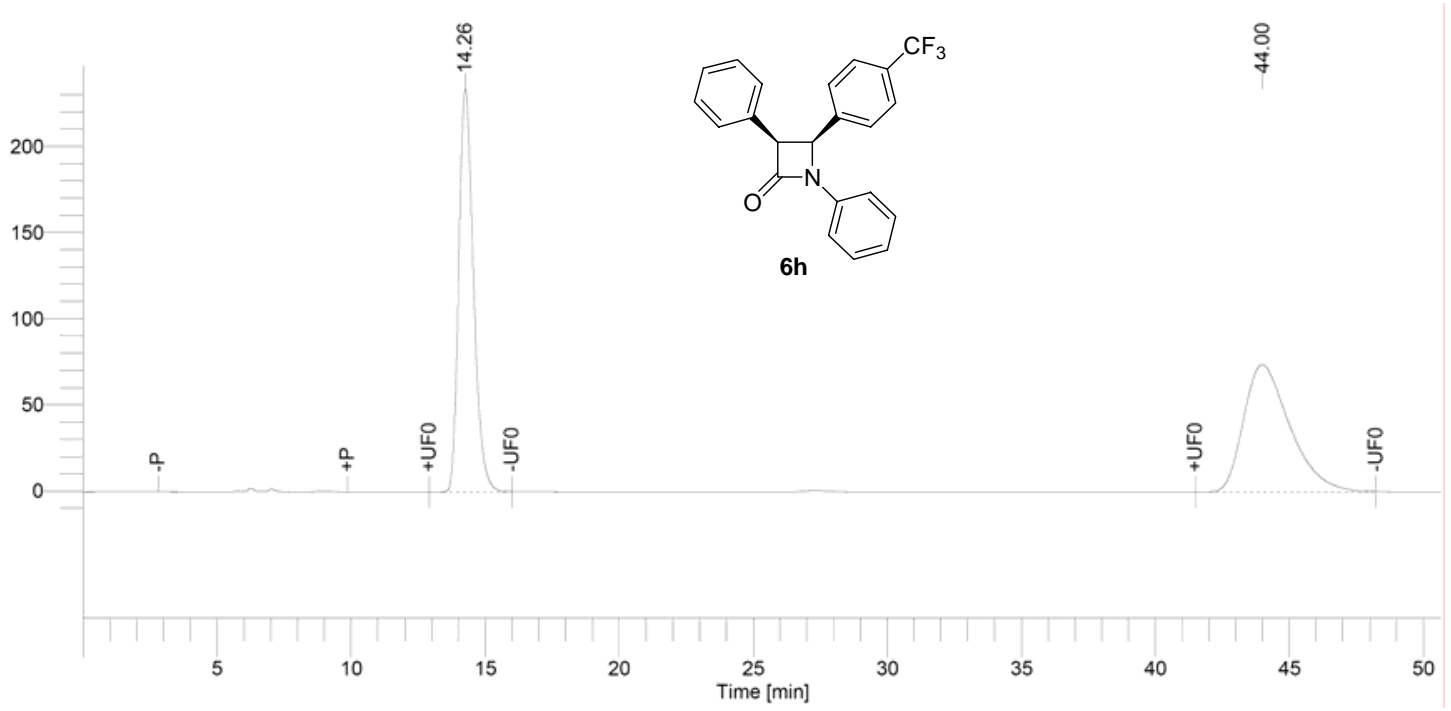

\begin{tabular}{|c|c|c|c|c|c|c|}
\hline $\begin{array}{l}\text { Time } \\
\text { [min] }\end{array}$ & $\begin{array}{c}\text { Area } \\
{\left[\mathrm{uV}^{*} \mathrm{sec}\right]}\end{array}$ & $\begin{array}{l}\text { Height } \\
{[\mathrm{uV}]}\end{array}$ & $\begin{array}{l}\text { Area } \\
{[\%]}\end{array}$ & $\begin{array}{c}\text { Norm. Area } \\
{[\%]}\end{array}$ & $B L$ & $\begin{array}{c}\text { Adjusted } \\
\text { Amount }\end{array}$ \\
\hline \multirow{3}{*}{$\begin{array}{l}14.258 \\
43.997\end{array}$} & 17318063.84 & 455082.79 & 50.09 & 50.09 & \multirow{3}{*}{$\begin{array}{l}\text { MM } \\
\text { MM }\end{array}$} & 17.3181 \\
\hline & 17254070.93 & 143673.01 & 49.91 & 49.91 & & 17.2541 \\
\hline & 4572134.77 & 598755.80 & 100.00 & 100.00 & & 34.5721 \\
\hline
\end{tabular}

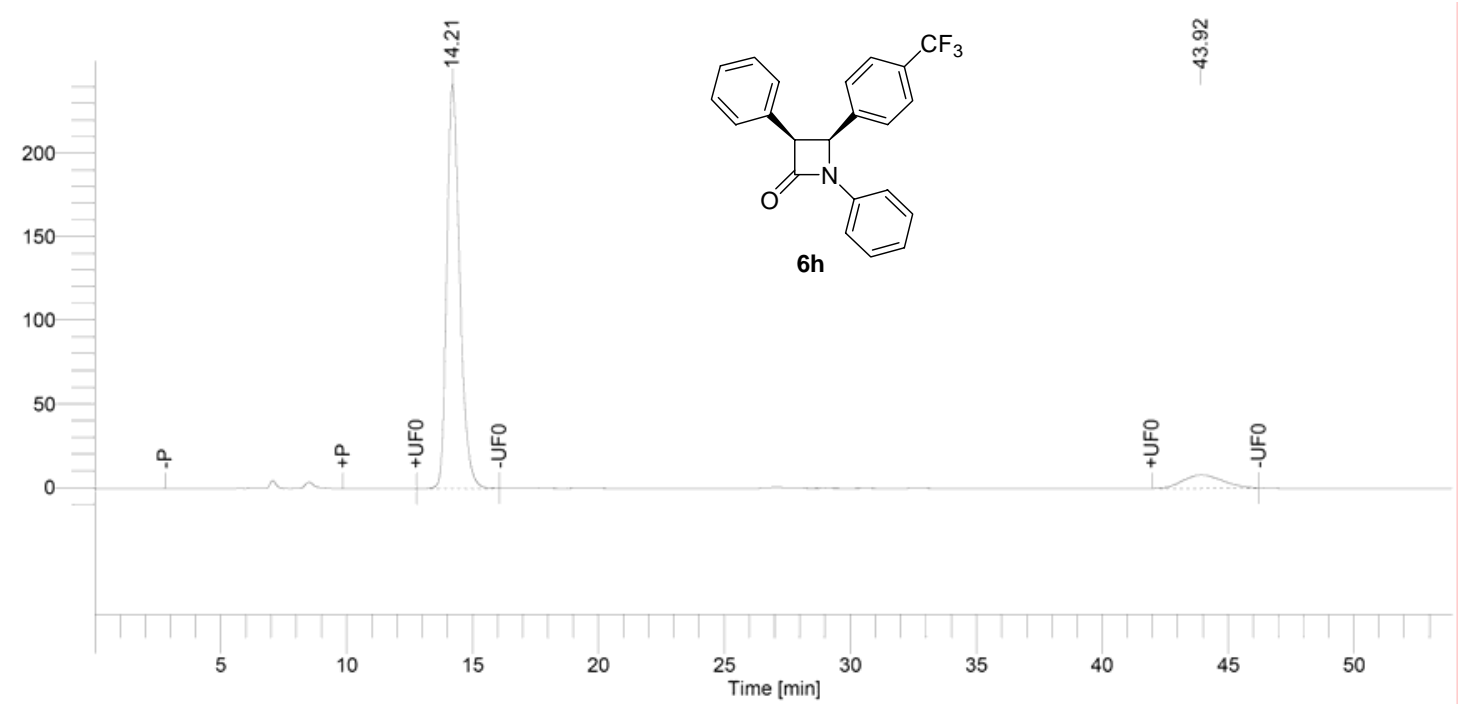

\begin{tabular}{|c|c|c|c|c|c|c|}
\hline $\begin{array}{l}\text { Time } \\
\text { [min] }\end{array}$ & $\begin{array}{c}\text { Area } \\
{\left[\mathrm{uV}^{*} \mathrm{sec}\right]}\end{array}$ & $\begin{array}{l}\text { Height } \\
{[\mathrm{uV}]}\end{array}$ & $\begin{array}{l}\text { Area } \\
{[\%]}\end{array}$ & $\begin{array}{c}\text { Norm. Area } \\
{[\%]}\end{array}$ & $\mathrm{BL}$ & $\begin{array}{c}\text { Adjusted } \\
\text { Amount }\end{array}$ \\
\hline \multirow{3}{*}{$\begin{array}{l}14.205 \\
43.920\end{array}$} & 16834563.97 & 470227.25 & 90.86 & 90.86 & MM & 16.8346 \\
\hline & 1693434.03 & 15533.27 & 9.14 & 9.14 & MM & 1.6934 \\
\hline & 00 & .52 & 0 & 100.00 & & 18.528 \\
\hline
\end{tabular}




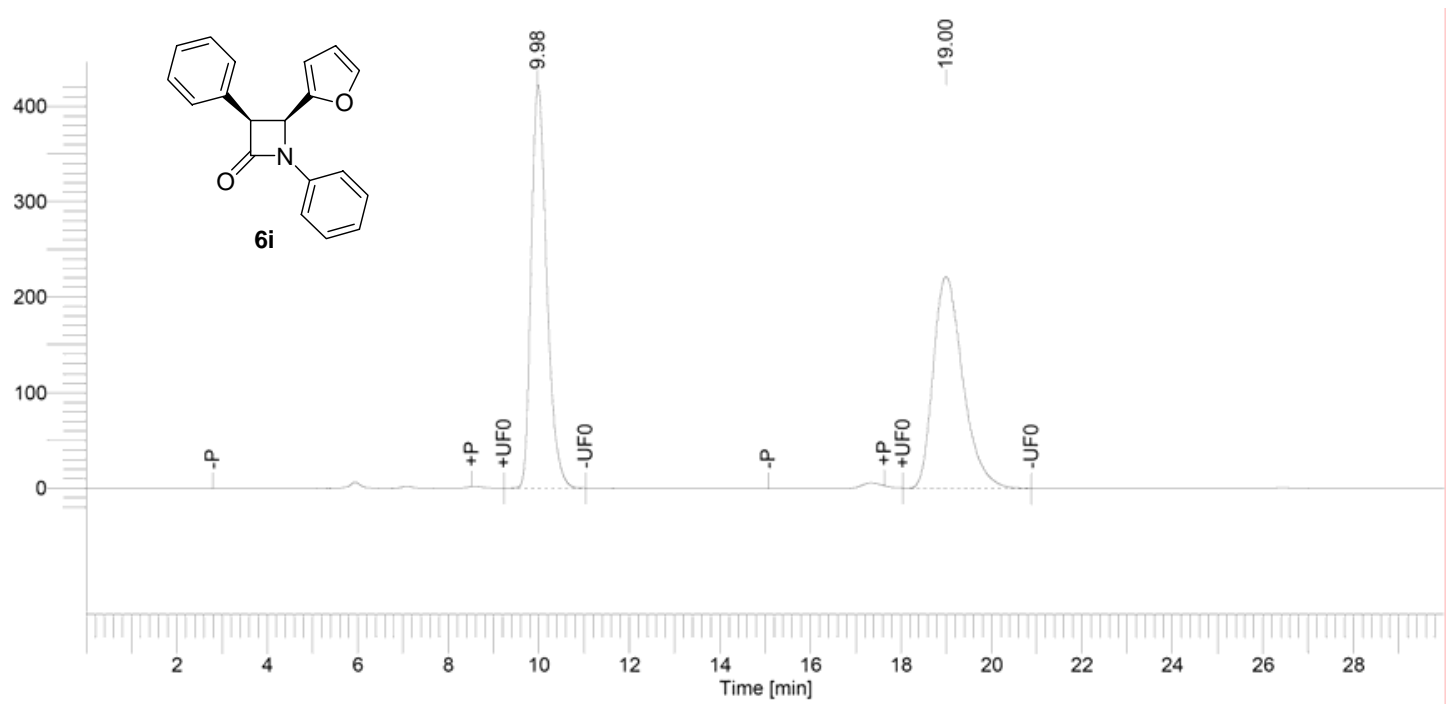

\begin{tabular}{|c|c|c|c|c|c|c|}
\hline $\begin{array}{l}\text { Time } \\
\text { [min] }\end{array}$ & $\begin{array}{c}\text { Area } \\
{\left[\mathrm{uV}^{*} \mathrm{sec}\right]}\end{array}$ & $\begin{array}{l}\text { Height } \\
{[\mathrm{uV}]}\end{array}$ & $\begin{array}{l}\text { Area } \\
{[\%]}\end{array}$ & $\begin{array}{c}\text { Norm. Area } \\
{[\%]}\end{array}$ & $B L$ & $\begin{array}{c}\text { Adjusted } \\
\text { Amount }\end{array}$ \\
\hline 9.980 & 19651747.27 & 821624.39 & 50.05 & 50.05 & MM & 19.65 \\
\hline 18.995 & 19612682.41 & 430469.81 & 49.95 & 49.95 & MM & 19.61 \\
\hline
\end{tabular}

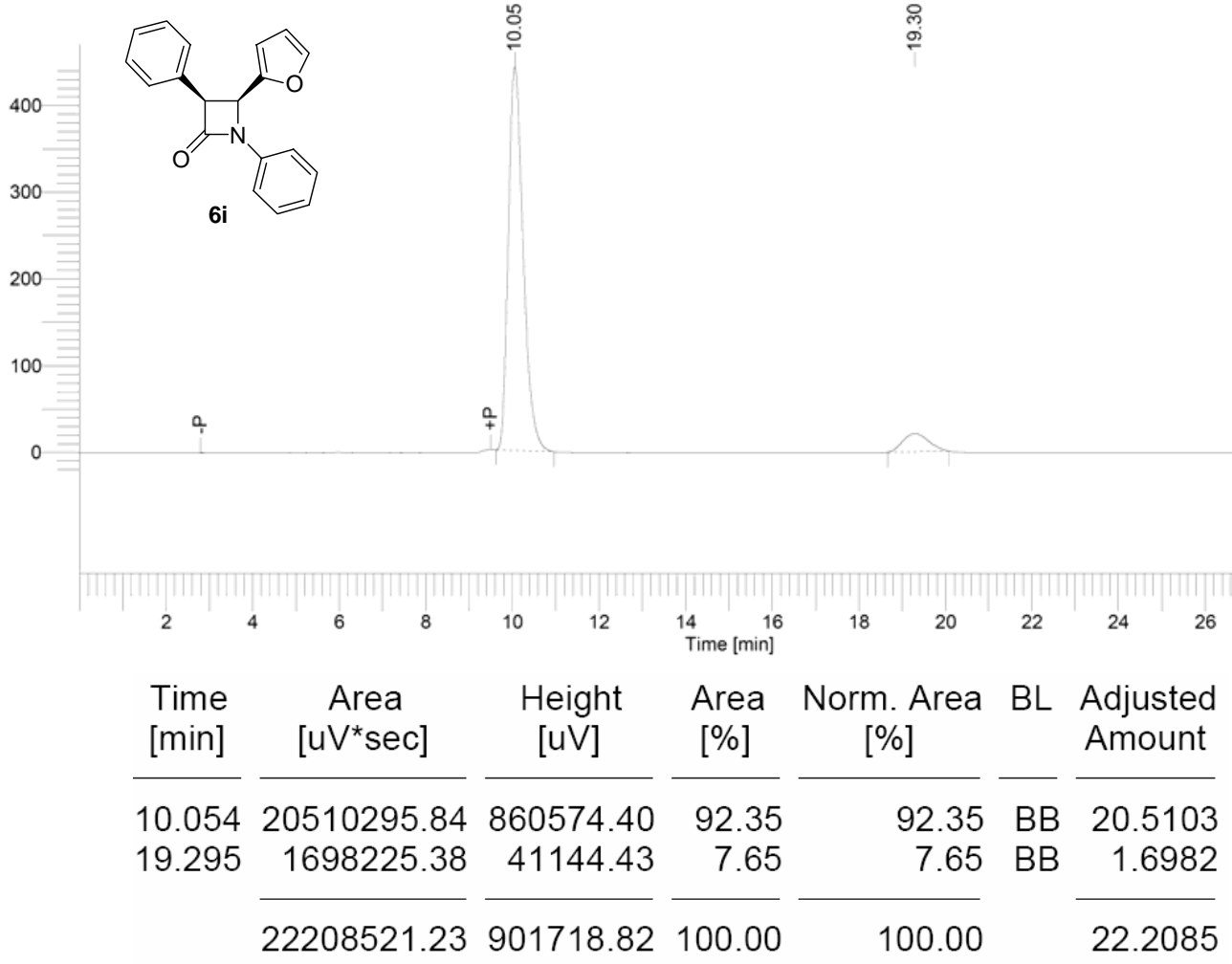




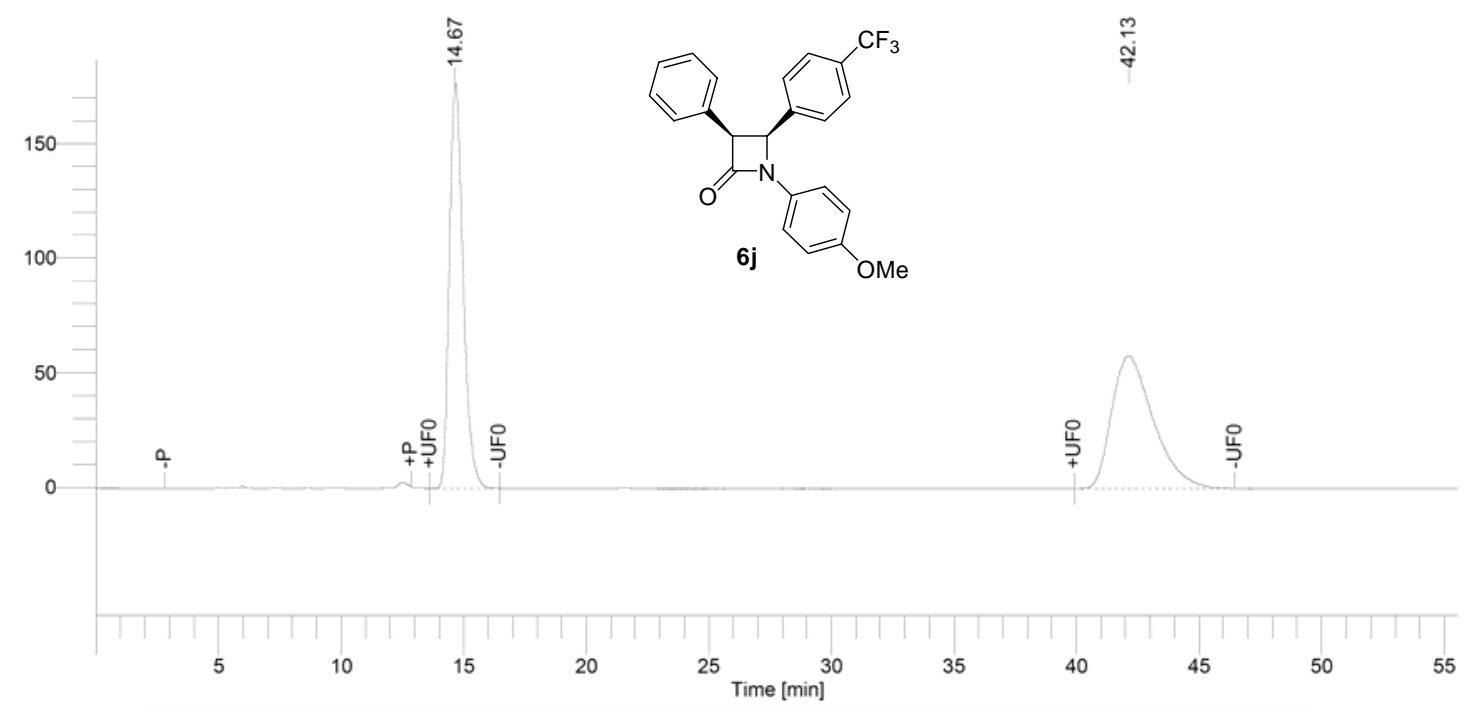

\begin{tabular}{|c|c|c|c|c|c|c|}
\hline $\begin{array}{l}\text { Time } \\
\text { [min] }\end{array}$ & $\begin{array}{c}\text { Area } \\
{\left[\mathrm{uV}^{*} \mathrm{sec}\right]}\end{array}$ & $\begin{array}{l}\text { Height } \\
{[\mathrm{uV}]}\end{array}$ & $\begin{array}{l}\text { Area } \\
{[\%]}\end{array}$ & $\begin{array}{c}\text { Norm. Area } \\
{[\%]}\end{array}$ & $\mathrm{BL}$ & $\begin{array}{l}\text { Adjusted } \\
\text { Amount }\end{array}$ \\
\hline 14.668 & 133909 & 344165.44 & 50.02 & 50.02 & MM & \\
\hline \multirow[t]{2}{*}{42.132} & 13382093.05 & 112362.94 & 49.98 & 49.98 & MM & 13.38 \\
\hline & 26773078.82 & 56528.38 & 100.00 & 100.00 & & 20 \\
\hline
\end{tabular}

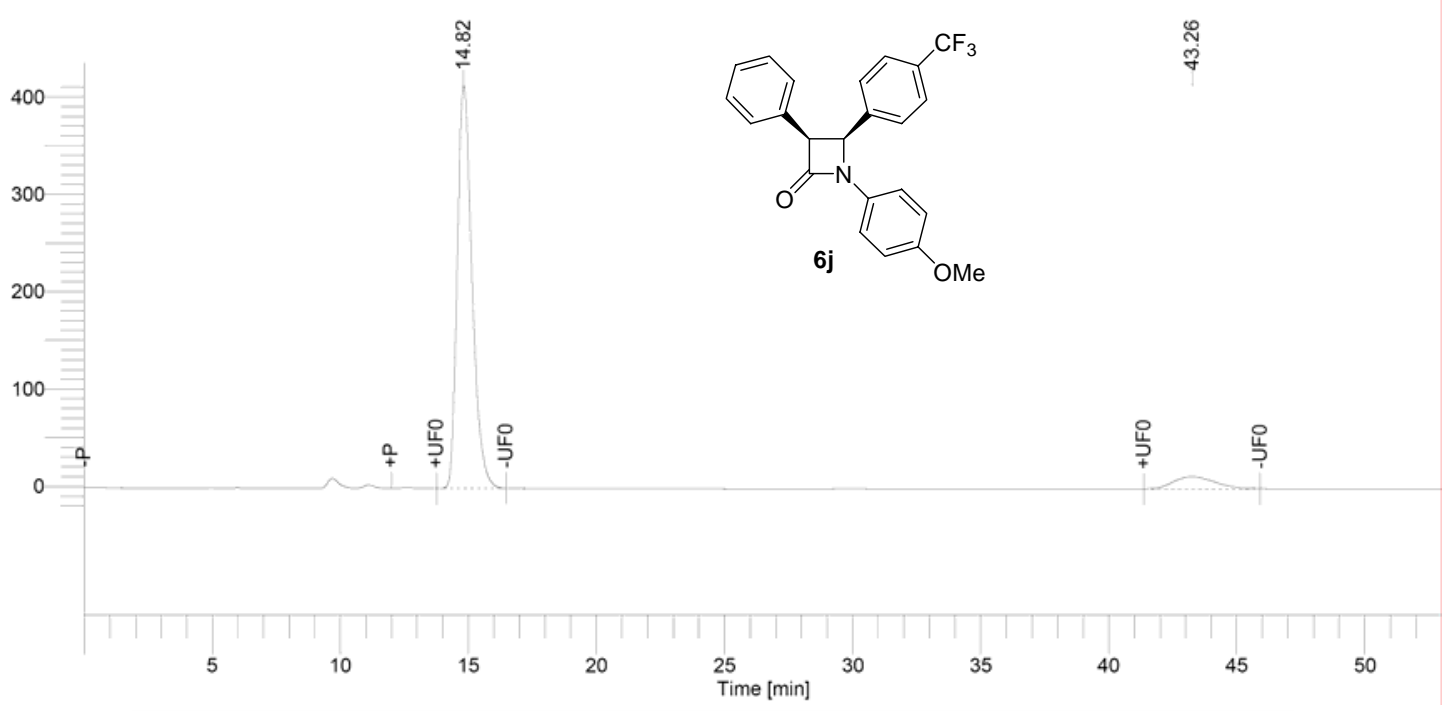

\begin{tabular}{|c|c|c|c|c|c|c|}
\hline $\begin{array}{l}\text { Time } \\
\text { [min] }\end{array}$ & $\begin{array}{c}\text { Area } \\
{\left[\mathrm{uV}^{*} \mathrm{sec}\right]}\end{array}$ & $\begin{array}{l}\text { Height } \\
{[\mathrm{uV}]}\end{array}$ & $\begin{array}{c}\text { Area } \\
{[\%]}\end{array}$ & $\begin{array}{c}\text { Norm. Area } \\
{[\%]}\end{array}$ & $\mathrm{BL}$ & $\begin{array}{l}\text { Adjusted } \\
\text { Amount }\end{array}$ \\
\hline \multirow{3}{*}{$\begin{array}{l}14.818 \\
43.260\end{array}$} & 32326952.03 & 803594.37 & 92.11 & 92.11 & MM & 32.32 \\
\hline & 2768211.34 & 24712.28 & 7.89 & 7.89 & MM & 2.768 \\
\hline & 37 & 65 & 100.00 & 100.00 & & 95 \\
\hline
\end{tabular}




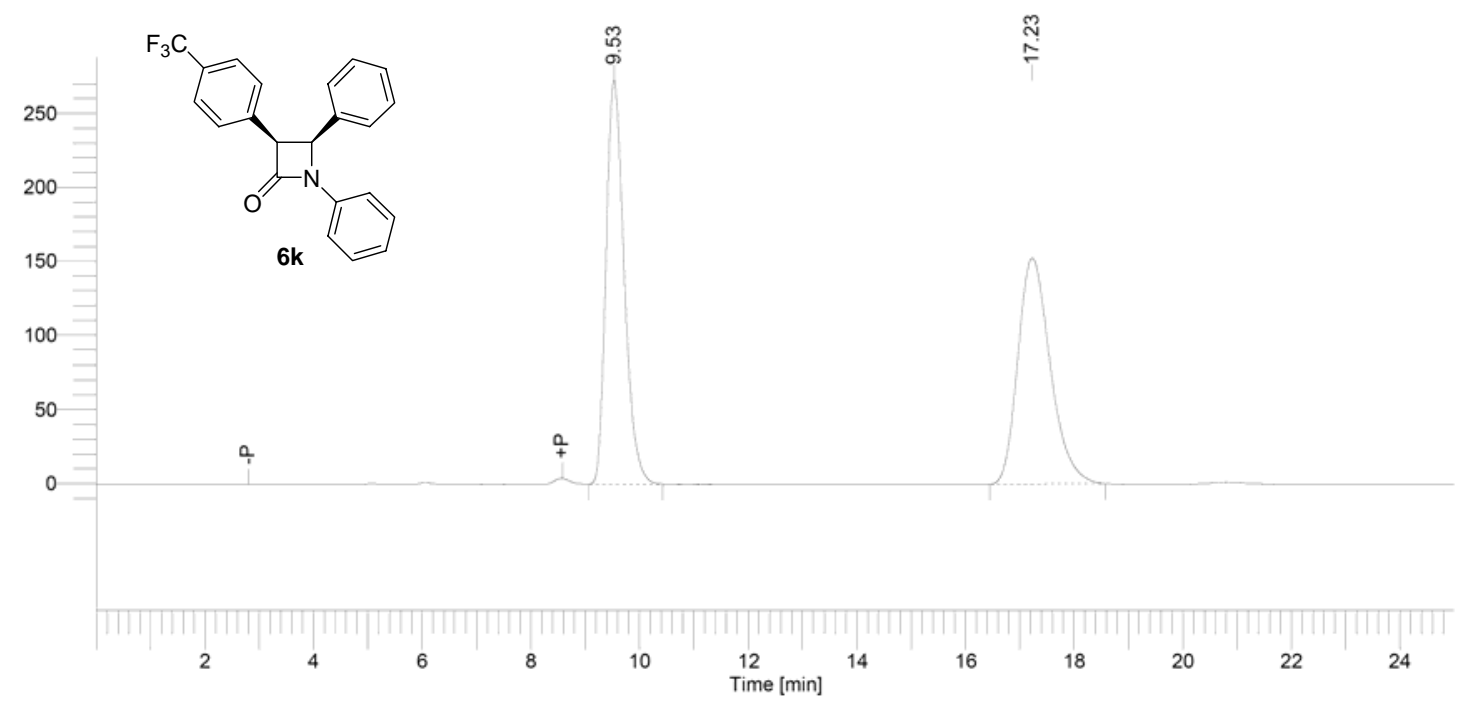

\begin{tabular}{|c|c|c|c|c|c|c|}
\hline $\begin{array}{l}\text { Time } \\
\text { [min] }\end{array}$ & $\begin{array}{c}\text { Area } \\
{\left[\mathrm{uV}^{*} \mathrm{sec}\right]}\end{array}$ & $\begin{array}{l}\text { Height } \\
{[\mathrm{uV}]}\end{array}$ & $\begin{array}{l}\text { Area } \\
{[\%]}\end{array}$ & $\begin{array}{c}\text { Norm. Area } \\
{[\%]}\end{array}$ & $\mathrm{BL}$ & $\begin{array}{c}\text { Adjusted } \\
\text { Amount }\end{array}$ \\
\hline \multirow{3}{*}{$\begin{array}{r}9.530 \\
17.229\end{array}$} & 12309 & 530408.59 & 50.04 & 50.04 & BB & 12.3097 \\
\hline & 12287981.39 & 296485.68 & 49.96 & 49.96 & BB & 12.2880 \\
\hline & 24597641.60 & 26894.27 & 100.00 & 100.00 & & 24.597 \\
\hline
\end{tabular}

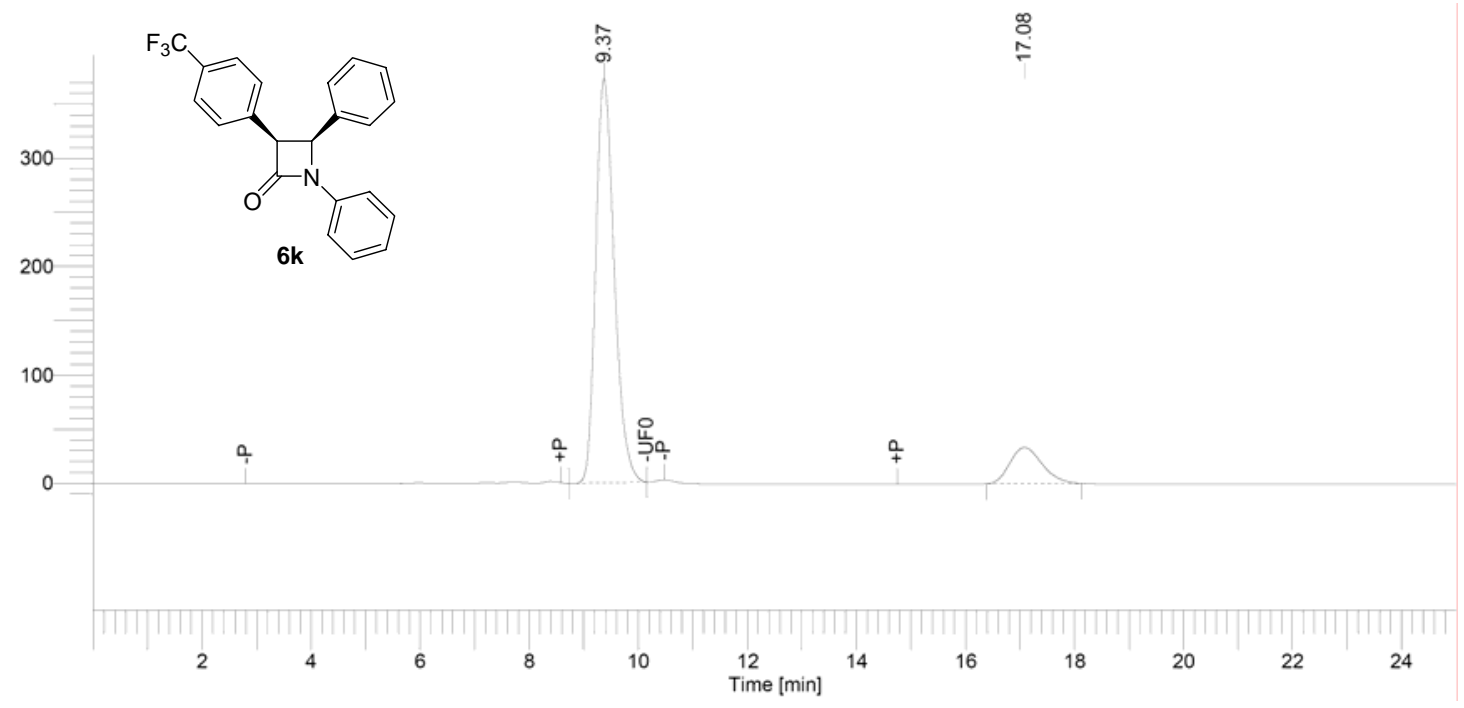

\begin{tabular}{|c|c|c|c|c|c|c|}
\hline $\begin{array}{l}\text { Time } \\
\text { [min] }\end{array}$ & $\begin{array}{c}\text { Area } \\
{\left[\mathrm{uV}^{*} \mathrm{sec}\right]}\end{array}$ & $\begin{array}{l}\text { Height } \\
{[\mathrm{uV}]}\end{array}$ & $\begin{array}{c}\text { Area } \\
{[\%]}\end{array}$ & $\begin{array}{c}\text { Norm. Area } \\
{[\%]}\end{array}$ & $B L$ & $\begin{array}{l}\text { Adjusted } \\
\text { Amount }\end{array}$ \\
\hline \multirow{3}{*}{$\begin{array}{r}9.370 \\
17.082\end{array}$} & 17148194.97 & 726107.26 & 86.39 & 86.39 & MM & 17.1482 \\
\hline & 2701824.45 & 65469.44 & 13.61 & 13.61 & BB & 2.701 \\
\hline & 198500 & .70 & .00 & 100.00 & & 19 \\
\hline
\end{tabular}




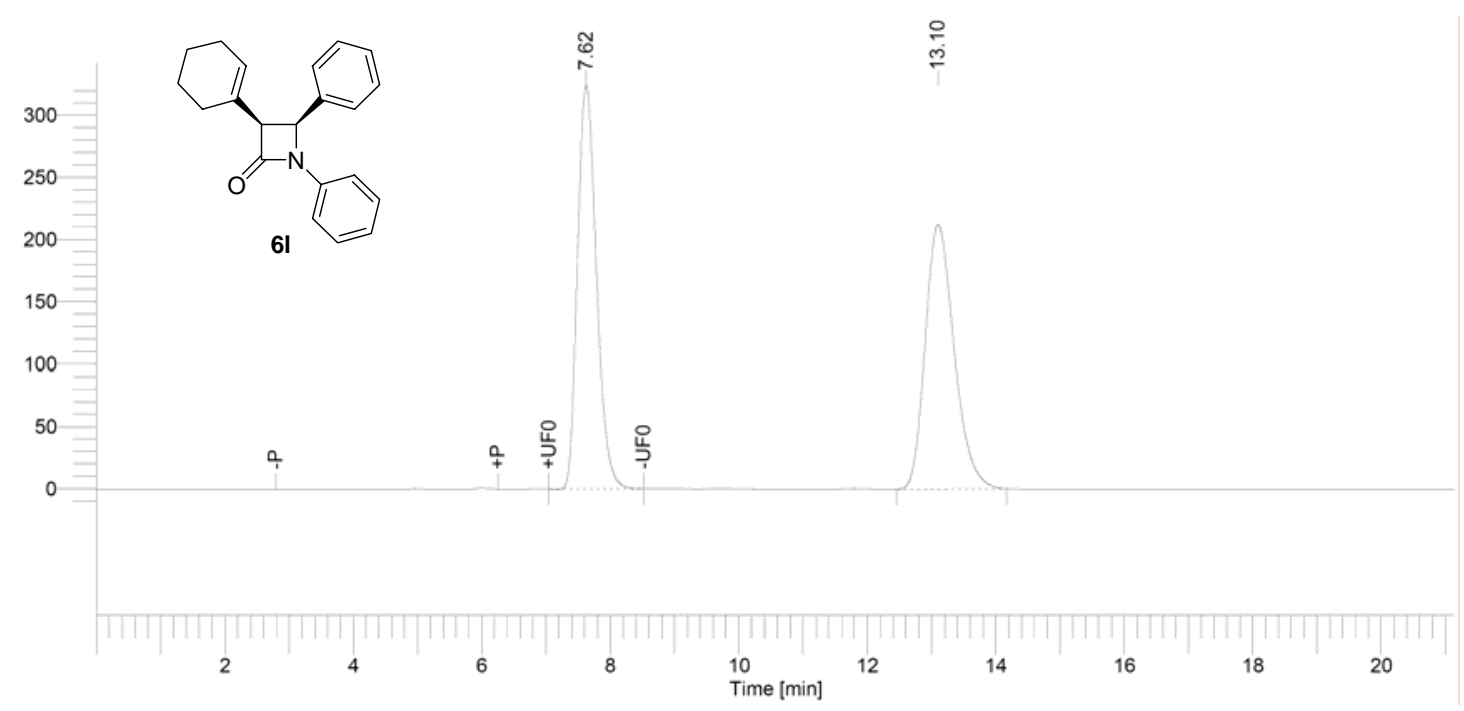

\begin{tabular}{|c|c|c|c|c|c|c|}
\hline $\begin{array}{l}\text { Time } \\
\text { [min] }\end{array}$ & $\begin{array}{c}\text { Area } \\
{\left[\mathrm{uV}^{*} \mathrm{sec}\right]}\end{array}$ & $\begin{array}{l}\text { Height } \\
{[\mathrm{uV}]}\end{array}$ & $\begin{array}{c}\text { Area } \\
{[\%]}\end{array}$ & $\begin{array}{c}\text { Norm. Area } \\
{[\%]}\end{array}$ & $B L$ & $\begin{array}{l}\text { Adjusted } \\
\text { Amount }\end{array}$ \\
\hline \multirow{3}{*}{$\begin{array}{r}7.623 \\
13.102\end{array}$} & 12922773.44 & 6297 & 49.99 & 49.99 & MM & 12.922 \\
\hline & 12930138.73 & 413164.55 & 50.01 & 50.01 & BB & 12.93 \\
\hline & 17 & $1.04 e+06$ & 100.00 & 100.00 & & 52 \\
\hline
\end{tabular}

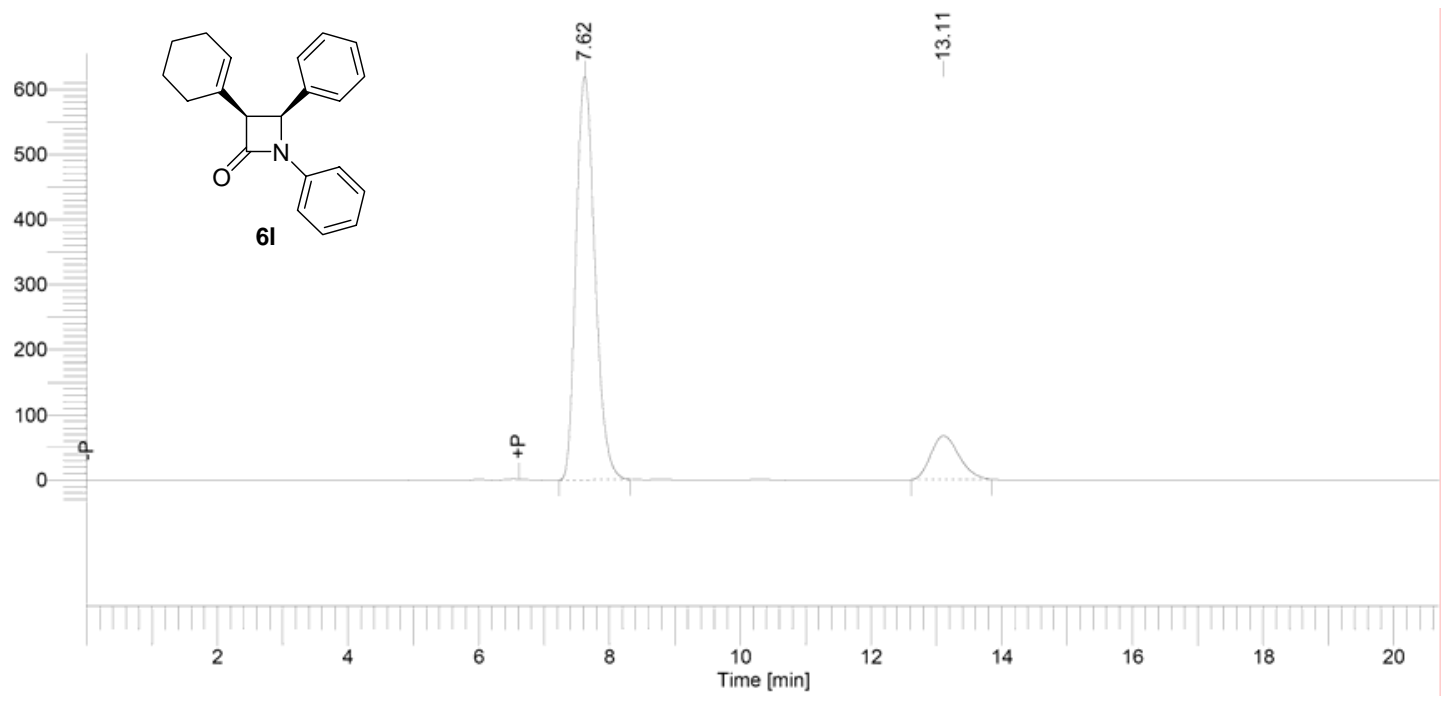

\begin{tabular}{|c|c|c|c|c|c|c|}
\hline $\begin{array}{l}\text { Time } \\
\text { [min] }\end{array}$ & $\begin{array}{c}\text { Area } \\
{\left[\mathrm{uV}^{*} \mathrm{sec}\right]}\end{array}$ & $\begin{array}{l}\text { Height } \\
{[\mathrm{uV}]}\end{array}$ & $\begin{array}{l}\text { Area } \\
{[\%]}\end{array}$ & $\begin{array}{c}\text { Norm. Area } \\
{[\%]}\end{array}$ & $B L$ & $\begin{array}{l}\text { Adjusted } \\
\text { Amount }\end{array}$ \\
\hline \multirow{3}{*}{$\begin{array}{r}7.619 \\
13.109\end{array}$} & 24242364.86 & $1.20 \mathrm{e}+06$ & 85.91 & 85.91 & BB & 24.2424 \\
\hline & 3976118.74 & 131891.10 & 14.09 & 14.09 & $\mathrm{BB}$ & 3.9761 \\
\hline & 282184 & $1.34 \mathrm{e}+06$ & 100.00 & 100.00 & & 28.2185 \\
\hline
\end{tabular}




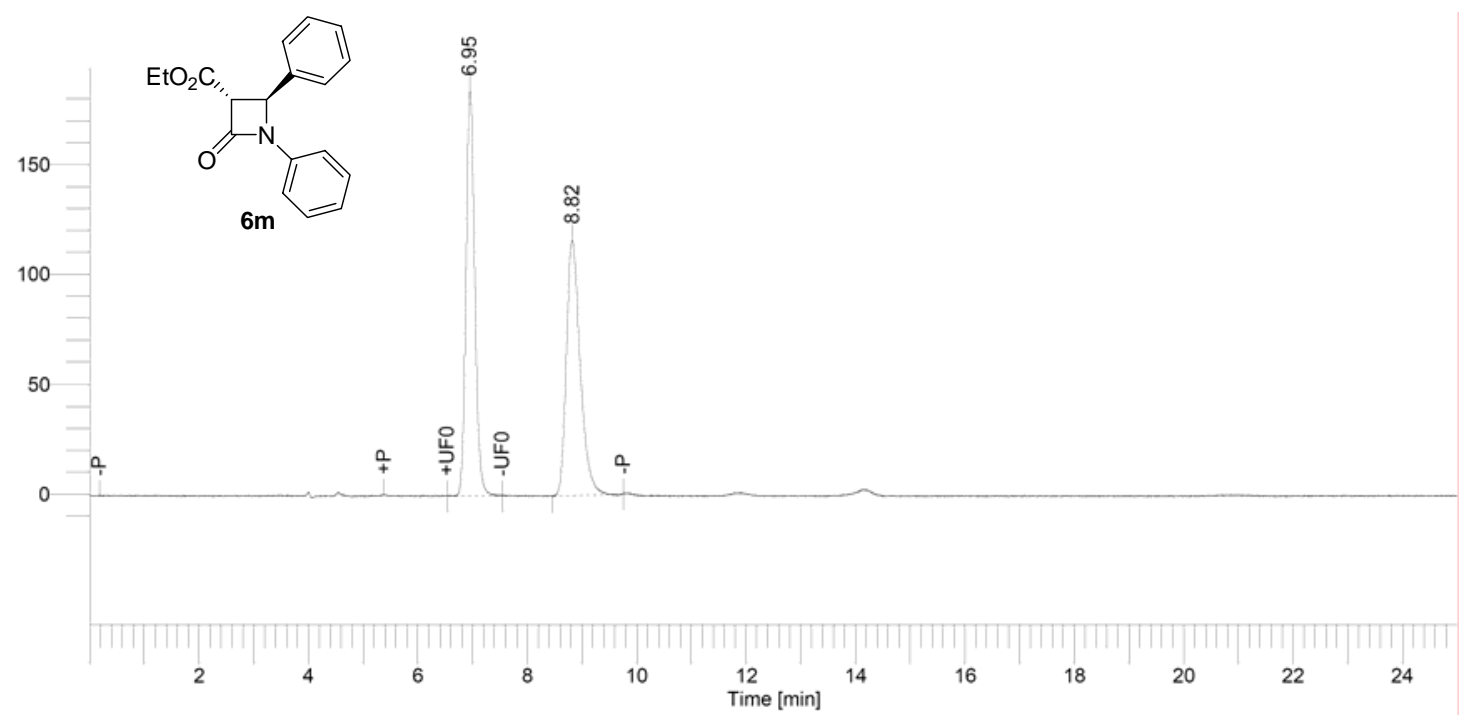

\begin{tabular}{|c|c|c|c|c|c|c|}
\hline $\begin{array}{l}\text { Time } \\
\text { [min] }\end{array}$ & $\begin{array}{c}\text { Area } \\
{\left[\mathrm{uV}^{*} \mathrm{sec}\right]}\end{array}$ & $\begin{array}{l}\text { Height } \\
{[\mathrm{uV}]}\end{array}$ & $\begin{array}{c}\text { Area } \\
{[\%]}\end{array}$ & $\begin{array}{c}\text { Norm. Area } \\
{[\%]}\end{array}$ & $\mathrm{BL}$ & $\begin{array}{c}\text { Adjusted } \\
\text { Amount }\end{array}$ \\
\hline 6.948 & 2068795.71 & 184132.30 & 50.29 & 50.29 & MM & 2.0688 \\
\hline \multirow[t]{2}{*}{8.822} & 2044704.27 & 116163.96 & 49.71 & 49.71 & ${ }^{*} \mathrm{BB}$ & 2.0447 \\
\hline & 4113499.98 & 300296.25 & 100.00 & 100.00 & & 1135 \\
\hline
\end{tabular}

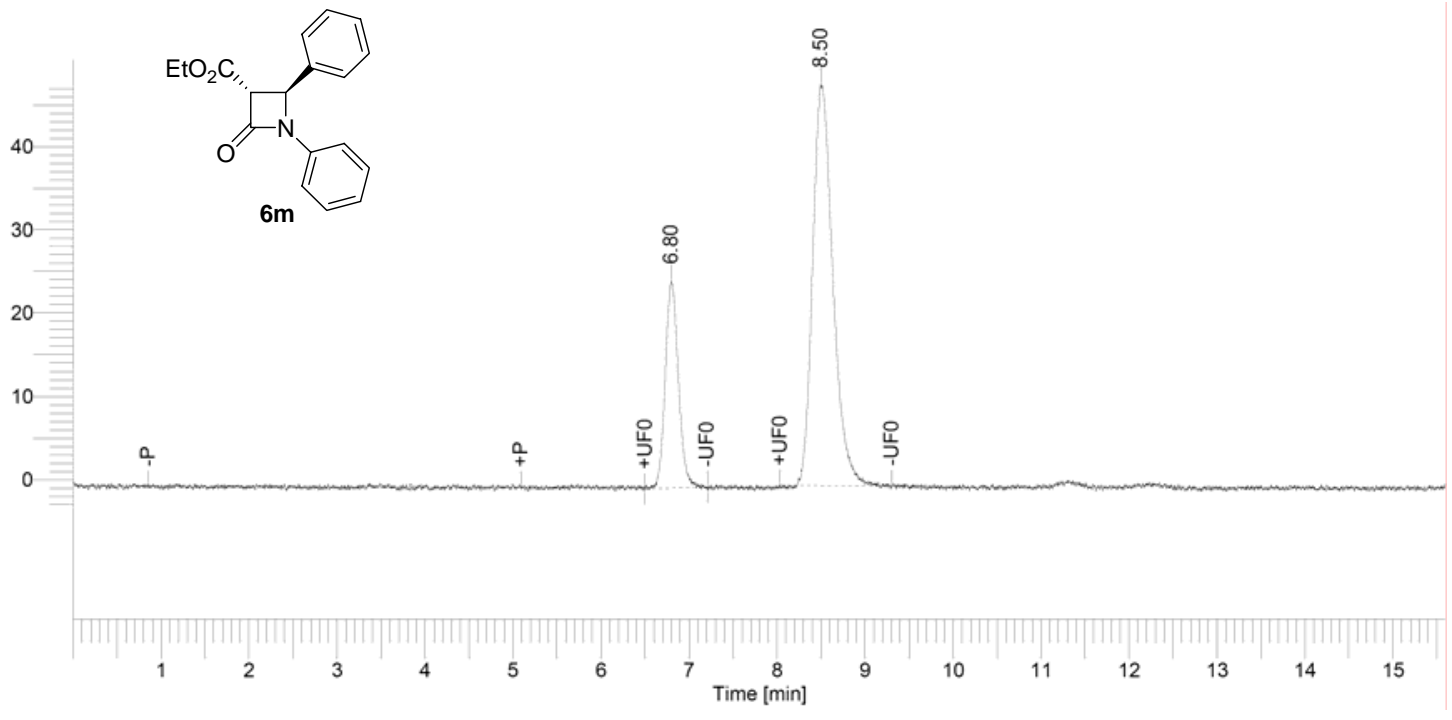

\begin{tabular}{|c|c|c|c|c|c|c|}
\hline $\begin{array}{l}\text { Time } \\
\text { [min] }\end{array}$ & $\begin{array}{c}\text { Area } \\
{\left[\mathrm{uV}^{*} \text { sec }\right]}\end{array}$ & $\begin{array}{l}\text { Height } \\
{[\mathrm{uV}]}\end{array}$ & $\begin{array}{l}\text { Area } \\
{[\%]}\end{array}$ & $\begin{array}{c}\text { Norm. Area } \\
{[\%]}\end{array}$ & $\mathrm{BL}$ & $\begin{array}{c}\text { Adjusted } \\
\text { Amount }\end{array}$ \\
\hline 6.798 & 257886.37 & 24955.76 & 25.02 & 25.02 & MM & 0.2579 \\
\hline 8.497 & 772845.46 & 48308.98 & 74.98 & 74.98 & MM & 0.7728 \\
\hline
\end{tabular}




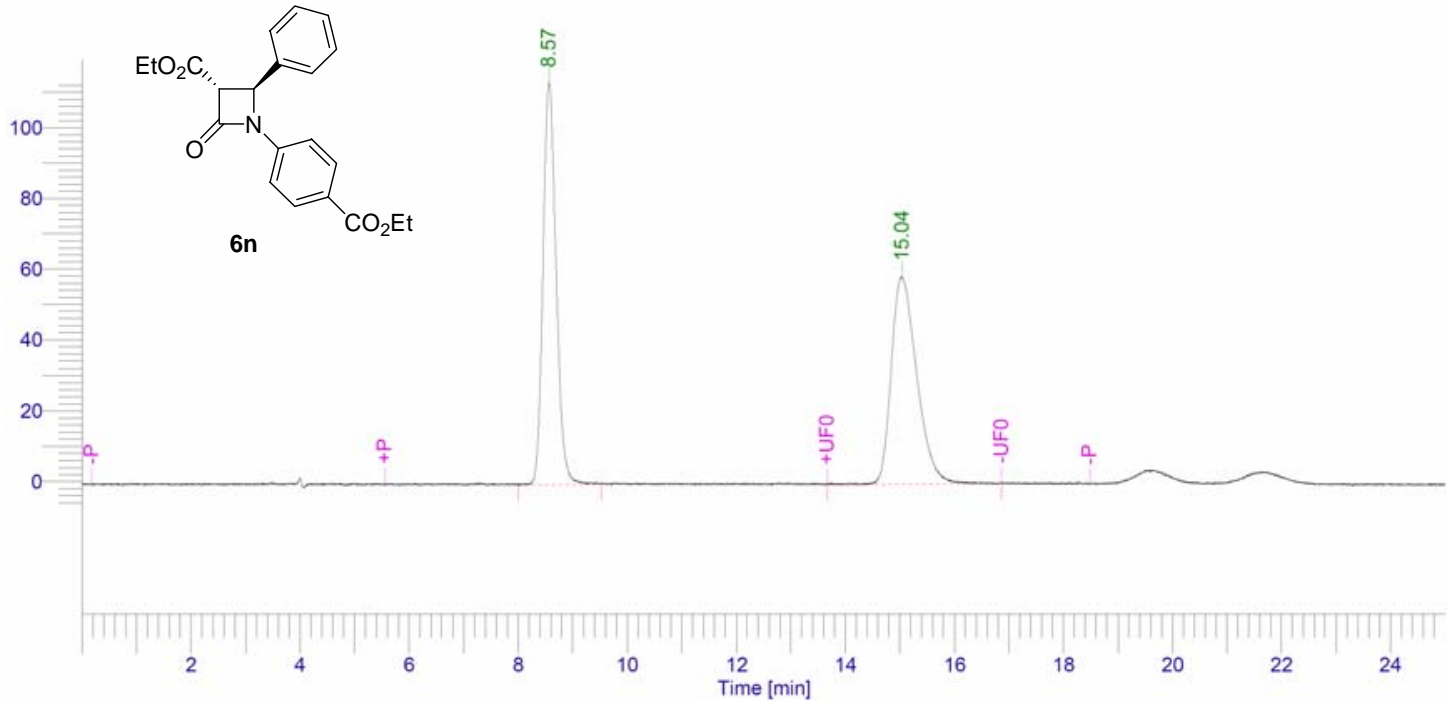

Time Area Height Area Norm. Area BL Adjusted

\begin{tabular}{|c|c|c|c|c|c|c|}
\hline [min] & {$\left[u V^{*} \mathrm{sec}\right]$} & {$[\mathrm{uV}]$} & {$[\%]$} & {$[\%]$} & & Amount \\
\hline 8.566 & 1910751.98 & 113541.05 & 50.11 & 50.11 & \multirow{3}{*}{$\begin{array}{l}\text { BB } \\
\text { MM }\end{array}$} & 1.9108 \\
\hline \multirow{2}{*}{15.038} & 1902223.19 & 58470.15 & 49.89 & 49.89 & & 1.9022 \\
\hline & 3812975.17 & 2011.20 & 0. & 100.00 & & 3.8 \\
\hline
\end{tabular}

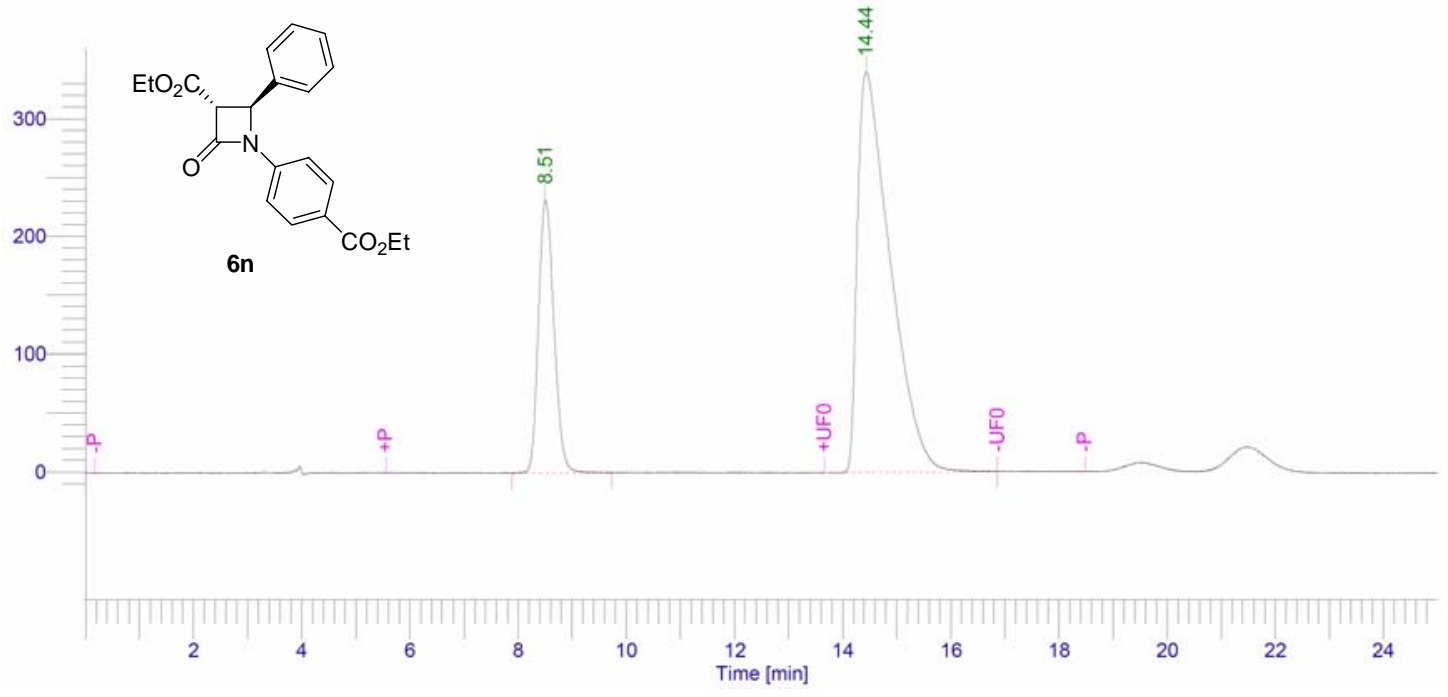

\begin{tabular}{|c|c|c|c|c|c|c|}
\hline $\begin{array}{l}\text { Time } \\
\text { [min] }\end{array}$ & $\begin{array}{c}\text { Area } \\
{\left[\mathrm{uV}^{*} \mathrm{sec}\right]}\end{array}$ & $\begin{array}{l}\text { Height } \\
{[\mathrm{uV}]}\end{array}$ & $\begin{array}{c}\text { Area } \\
{[\%]}\end{array}$ & $\begin{array}{c}\text { Norm. Area } \\
{[\%]}\end{array}$ & $\mathrm{BL}$ & $\begin{array}{c}\text { Adjusted } \\
\text { Amount }\end{array}$ \\
\hline \multirow{3}{*}{$\begin{array}{r}8.505 \\
14.435\end{array}$} & 4638793.75 & 231679.50 & 24.51 & 24.51 & BB & 4.6388 \\
\hline & 14287970.91 & 339889.30 & 75.49 & 75.49 & MM & 14.2880 \\
\hline & 18926764.66 & 571568.80 & 100.00 & 100.00 & & 18.9268 \\
\hline
\end{tabular}




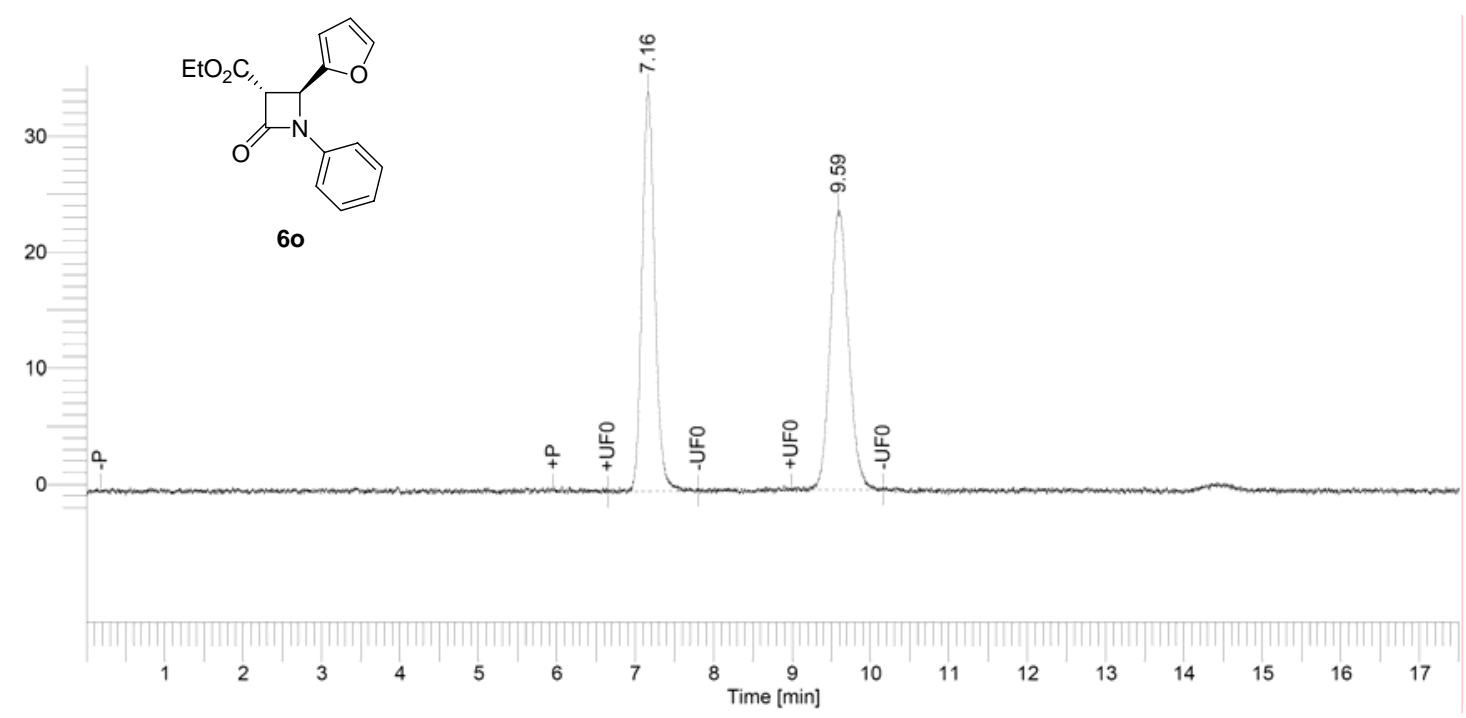

Time Area Height Area Norm. Area BL Adjusted

\begin{tabular}{|c|c|c|c|c|c|c|}
\hline$[\mathrm{min}]$ & {$\left[\mathrm{uV}^{*} \mathrm{sec}\right]$} & [uV] & [\%] & [\%] & & Amount \\
\hline .162 & 3904 & 34638.93 & 49.93 & 49.93 & MM & 0.3905 \\
\hline \multirow[t]{2}{*}{9.593} & 391578.26 & 24138.59 & 50.07 & 50.07 & MM & 0.3916 \\
\hline & 782055.79 & 58777.52 & 100.00 & 100.00 & & 0.1 \\
\hline
\end{tabular}

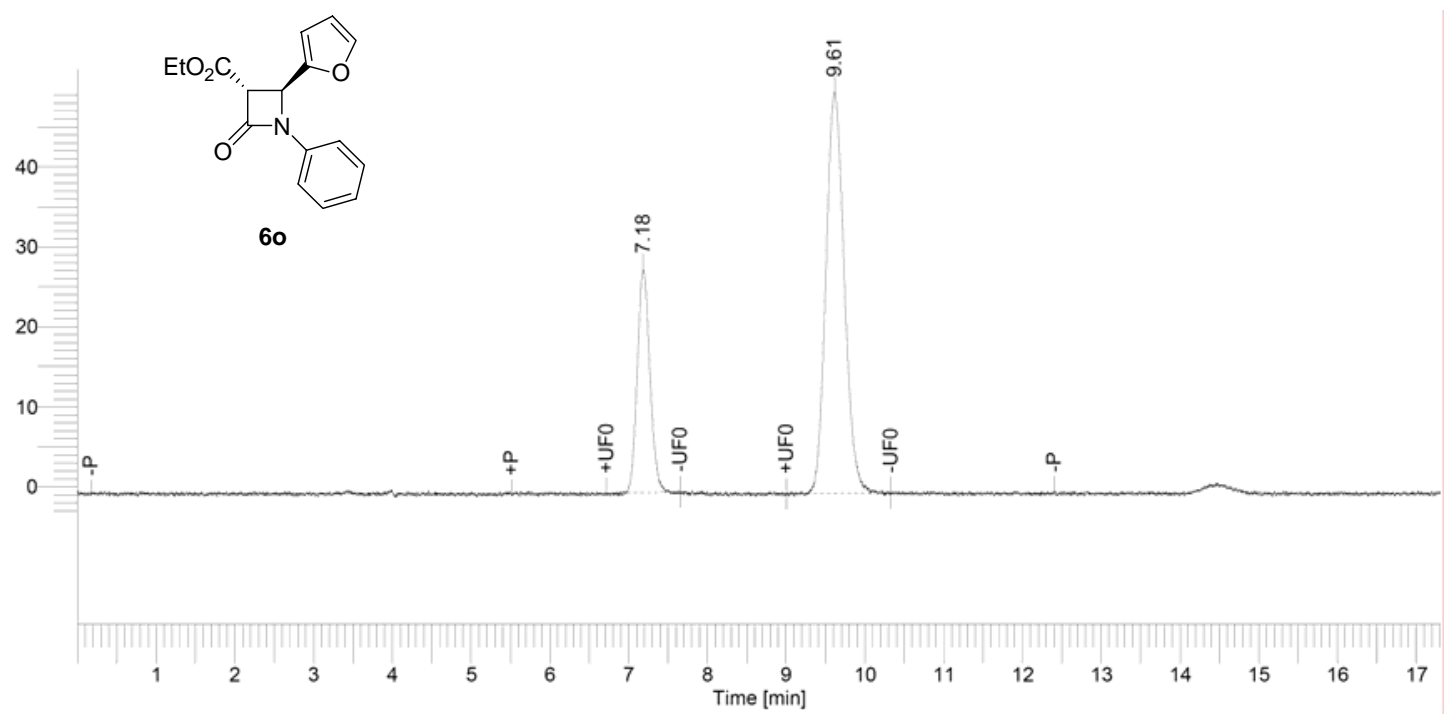

\begin{tabular}{|c|c|c|c|c|c|c|}
\hline $\begin{array}{l}\text { Time } \\
\text { [min] }\end{array}$ & $\begin{array}{c}\text { Area } \\
{\left[\mathrm{uV}^{*} \mathrm{sec}\right]}\end{array}$ & $\begin{array}{l}\text { Height } \\
{[\mathrm{uV}]}\end{array}$ & $\begin{array}{c}\text { Area } \\
{[\%]}\end{array}$ & $\begin{array}{c}\text { Norm. Area } \\
{[\%]}\end{array}$ & $\mathrm{BL}$ & $\begin{array}{l}\text { Adjusted } \\
\text { Amount }\end{array}$ \\
\hline 7.183 & 310570.34 & 27891.24 & 27.68 & 27.68 & MM & 0.3106 \\
\hline \multirow[t]{2}{*}{9.613} & 811567.62 & 50088.51 & 72.32 & 72.32 & MM & 0.8116 \\
\hline & .96 & 7979.75 & 100.00 & 100.00 & & 1.1221 \\
\hline
\end{tabular}

